\title{
To stay or not to stay : the assessment of inappropriate hospital stay
}

Citation for published version (APA):

Panis, L. J. G. (2004). To stay or not to stay : the assessment of inappropriate hospital stay. [Doctoral Thesis, Maastricht University]. Universiteit Maastricht. https://doi.org/10.26481/dis.20040415lp

Document status and date:

Published: 01/01/2004

DOI:

10.26481/dis.20040415lp

Document Version:

Publisher's PDF, also known as Version of record

\section{Please check the document version of this publication:}

- A submitted manuscript is the version of the article upon submission and before peer-review. There can be important differences between the submitted version and the official published version of record.

People interested in the research are advised to contact the author for the final version of the publication, or visit the DOI to the publisher's website.

- The final author version and the galley proof are versions of the publication after peer review.

- The final published version features the final layout of the paper including the volume, issue and page numbers.

Link to publication

\footnotetext{
General rights rights.

- You may freely distribute the URL identifying the publication in the public portal. please follow below link for the End User Agreement:

www.umlib.nl/taverne-license

Take down policy

If you believe that this document breaches copyright please contact us at:

repository@maastrichtuniversity.nl

providing details and we will investigate your claim.
}

Copyright and moral rights for the publications made accessible in the public portal are retained by the authors and/or other copyright owners and it is a condition of accessing publications that users recognise and abide by the legal requirements associated with these

- Users may download and print one copy of any publication from the public portal for the purpose of private study or research.

- You may not further distribute the material or use it for any profit-making activity or commercial gain

If the publication is distributed under the terms of Article $25 \mathrm{fa}$ of the Dutch Copyright Act, indicated by the "Taverne" license above, 


\title{
To stay or not to stay: The assessment of inappropriate hospital stay
}

\author{
PROEFSCHRIFT
}

ter verkrijging van de graad van doctor aan de universiteit Maastricht op gezag van de Rector Magnificus, Prof. Mr. G. P. M. F. Mols volgens het besluit van het College van Decanen, in het openbaar te verdedigen. op donderdag 15 aprill 2004 onn 12.00 uur

door

Lambert. Jean Gerard Gertrude Panis

geboren te Ḧasselt (B) op 5 april 1963 
Promotores:

Prof. dr. M. Prins

Prof. dr. P. Pop

Co-promotor:

Dr. F. Verheggen

Beoordelingscommissie: Prof. dr. G. van Merode (voorzitter)

Prof. dr. J. Carpay (Technische Universiteit Eindhoven)

Prof. dr. R. Grol (UMC St. Radboud Nijmegen)

Prof. dr. H. Hillen

Prof. dr. F. van Wijmen

ISBN $90-9017922-4$

Productic drukwerk: Datawyse, Maastricht

Ontwerp omslag: C. Amoldussen

2004, Lambert Panis. Lanaken, Belgie 
für Elice 



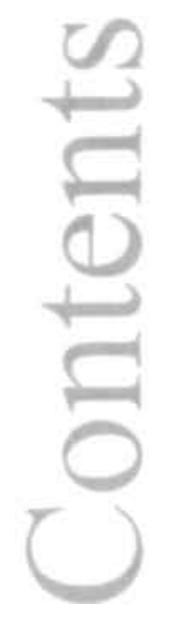

Chapter 1: Introduction.

$\begin{array}{lll}\text { Chapter 2: } & \text { The jumble of appropriateness. }\end{array}$

Chapter 3: $\quad$ Factors related to hospital stay. 25

Chapter 4: The need to proceed. 33

Chapter 5: $\quad$ To stay or not to stay. 51

Chapter 6: How inappropriate is appropriate stay? 69

$\begin{array}{ll}\text { Chapter 7: In search for appropriateness. } & 79\end{array}$

$\begin{array}{lll}\text { Chapter 8: } & \text { Predictors of inappropriate stay. } & 89\end{array}$

$\begin{array}{ll}\text { Chapter 9: } \quad \text { Flaws in patient flows. } & 05\end{array}$

Chapter 10: The impact of hospital discharge procedures. 119

$\begin{array}{ll}\text { Chapter 11: } & \text { General discussion. }\end{array}$

$\begin{array}{ll}\text { Summary } & 145\end{array}$

$\begin{array}{ll}\text { Resumé } & 151\end{array}$

$\begin{array}{lr}\text { Dankwoord } & 157\end{array}$

$\begin{array}{lr}\text { Curriculum Vitae } & 159\end{array}$ 



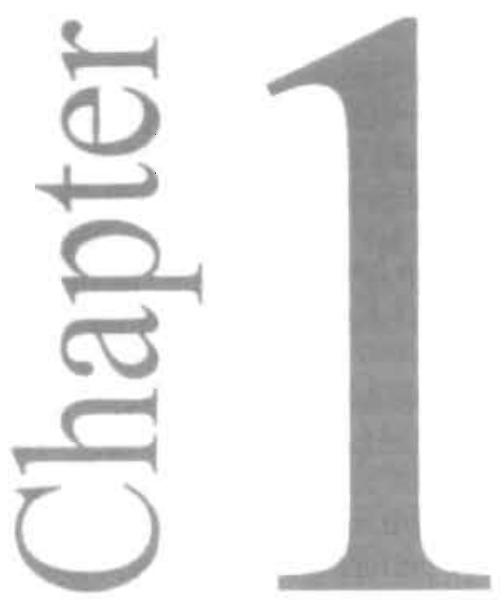

Introduction 


\section{Strains on modern health care}

Every year an enormous and growing amount of knowledge, procedures and techniques to improve health care becomes available.' Yet, all western industrialized countries are facing the challenge to provide a health care system that not only delivers up to date care of high quality, but that also must be affordable. New procedures and techniques usually create increased demands while financial resources are limited. Hence, choices have to be made in health care concerning the allocation of people, time, facilities, equipment and knowledge, even for interventions proven to be effective. Priorities have to be set to limit expenses.

At the same time, health care is facing demands for increased accountability. ${ }^{4}$ This means that reporting on the activities of health care organizations is required. ${ }^{5}$ To assess the achievement of goals and to compare quality of care between health care institutions, standards and criteria are needed. A standard is a generally accepted objective that can be used to assess individual levels of performance. Usually, such a standard is based on a rule or guideline supported by expert consensus, based on specific research or literature. Accountability refers to performance in health care organizations and to performance targets. Performance is the supply of health services that involve professional and patient oriented inputs and outcomes. To assess accountability, clinical performance indicators, such as protocols, evidence based practice, etc... have to be available and geared to real practice. ${ }^{6}$ A central feature of accountability is guaranteeing that appropriate services are provided in appropriate settings. Appropriate care can be defined as care that must -for reasons of ethics and medical necessity - be offered to the patient. ${ }^{7}$ An appropriate setting is a setting in which a efficient use of health care facilities match the patient's medical need. ${ }^{8}$ To reach this goal, criteria for appropriate care must be established, combining critical literature review with expert consensus. Subsequently performance has to be systematically assessed using these criteria of appropriateness. This assessment has to be methodologically sound and involve the opinions of the medical and nursing staff (fig 1).

Fig. I: Deming or Improvement Cycle

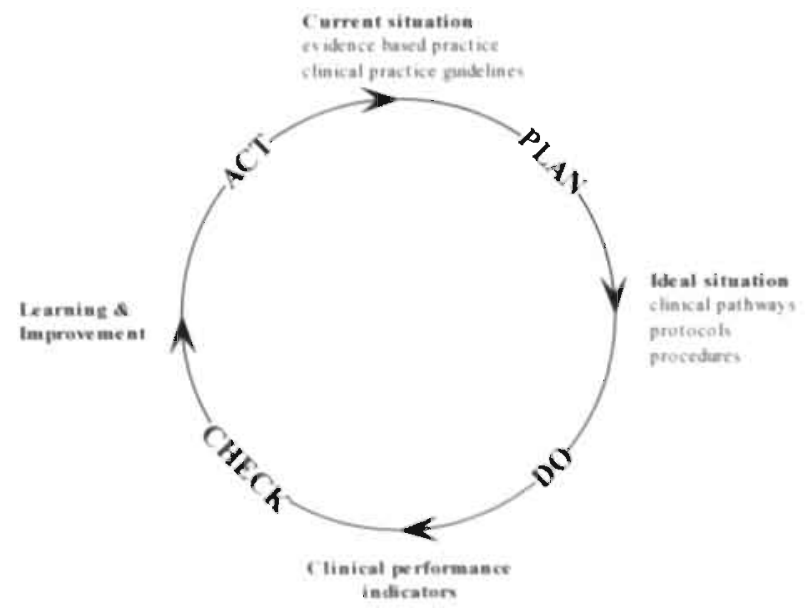


The increasing demands in face of limited resources, in combination with the need for accountability, puts hospitals under great pressure to improve the efficiency of internal operations, without sacrificing quality of care. Hence care has to be effective (the patient's condition has to be improved as much as possible), efficient (the best result has to be obtained with the fewest resources) and have the highest possible quality (the patient's preferences have to be combined with professional technology and procedures, avoiding errors, waste and delays).

Health care is a complex system. "Complex systems are best organized by their purposes. In case of health care this purpose must be seen through the eyes of the informed patient: "They give me exactly the help I want (and need) exactly when I want (and need) it." 10 The patient's need is to receive optimal health care, but changes to optimize health care are often difficult to implement or only introduced with large delays. Delays in implementation of innovation are often related to a lack of fit with the setting or the target group, to interference with structural, financial or organizational items, or to ineffective methods of implementation. "Consequently patients can be deprived of good health care, or they receive unnecessary, outmoded or even harmful care. There is evidence that within health care there is often an "underuse". "overuse" or even "misuse" of care. ${ }^{12-17}$ Although there is a growing consensus that health care quality should be improved (i.e., better effectiveness, efficiency, safety and patient orientation), there is no consensus on how this should be achieved. ${ }^{14.15}$ Furthermore, not all innovations are improvements. The Institute of Medicine stated in 'Crossing the Qualify Chasm' ${ }^{\text {in }}$ that health care systems should be reinvented to foster innovation and improve the delivery of care, in order to reach optimal health care (figure 2). This health care should be:

- Safe: ayoiding injuries to patients from the care that is intended to help them.

- Effective: providing services based on scientific knowledge to all who could benefit, and refraining from providing services to those not likely to benefit.

- Patient-centered: providing care that is respectful of and responsive to individual patient preferences, needs, and values, and ensuring that patient values guide all clinical decisions.

- Timely: reducing waits and sometimes-harmful delays for both those who receive and those who give care.

- Efficient: avoiding waste, including waste of equipment, supplies, ideas, and energy.

- Equitable: providing care that does not vary in quality because of personal characteristics such as gender, ethnicity, geographic location, and socioeconomic status.

The appropriateness of health care focuses on the safety and effectiveness of care, while the appropriateness of the health care setting concentrates on timeliness and efficiency of the provided care. 


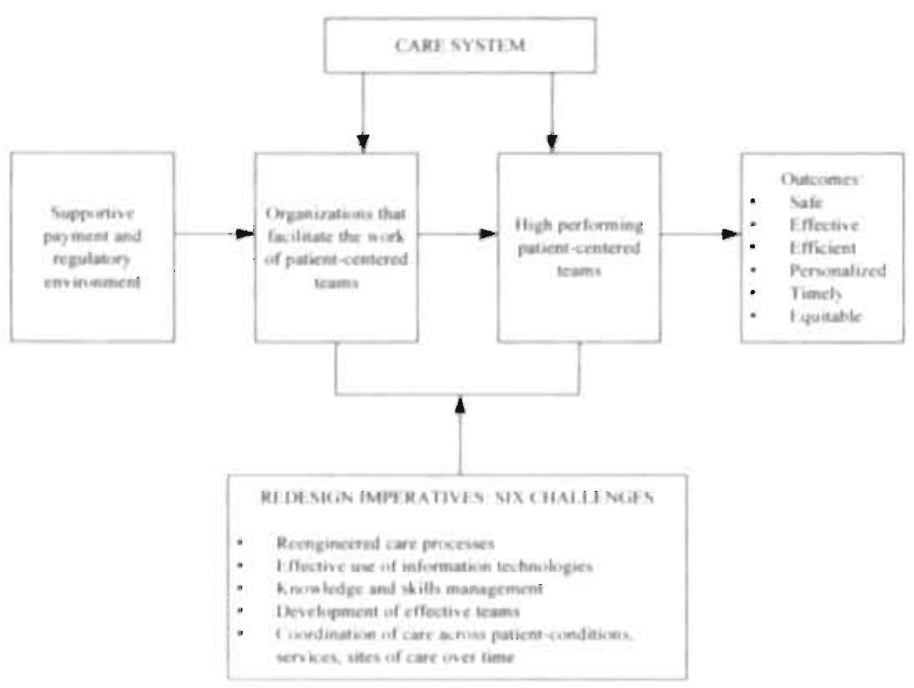

\section{Use of hospital facilities}

In an attempt to reduce the cost of health care, and among other cost containment actions, the number of hospital beds per 1000 inhabitants in the Netherlands has been decreased from 4.7 in 1985 to 3.5 in 2002 . ${ }^{17}$ Since such a reduction increases the pressure on the process of allocation of patients to the remaining beds, these beds have to be used as efficiently as possible. Inefficiency herein, affects the quality of hospital care and slows down the patient logistics, resulting in delays in patient tumover and longer waiting lists and waiting times. It should be realized that appropriate care not only concerns the medical intervention or procedures, but also the appropriateness of the setting in which that care is provided. If appropriate care increases and, consequently, inappropriate care decreases, the benefits to patients and society would be substantial. ${ }^{18}$ Appropriate hospital care can be seen as the match between the patient's clinical characteristics, the services required for this care and the setting in which this care is provided. Assuming that health care services are appropriate and provided in a technically correct way, they still can be provided in inappropriate settings. Reducing inappropriate hospital stay (IHS) -i.e. inappropriateness towards the setting in which health care is provided to the patient- can reduce costs and increase efficiency, mainaining the quality of care and preserving the access to that care by reserving hospital resources for those who need inpatient care. If a part of the health care is providied in an inappropriate setting, this can be seen not only as a waste of money but also as a wasie of quality. Consequently, reducing inappropriate stay could not only improve the quality of care, but also save money. If this assumption is true, reducing inappropriate stay is an approach to improve the quality of the current health care system. Consequently the question arises: "How to measure IHS?" and "What interventions to reduce IHS are needed?". 


\section{Research question}

\section{Aim}

In 1.994, the Peer-Review Committee of the University Hospital of Maastricht initiated a study ${ }^{19}$ into a more efficient use of hospital beds. The aim was to have a reliable, valid and applicable monitoring tool, for use by physicians and nurses at ward level, to make sound decisions on whether a particular patient's stay is appropriate or not. To evaluate the efficiency of hospital utilization, tools to assess the appropriateness of hospital stay were already available, but not designed for use in the Dutch health care system. Thus, it was decided to evaluate whether existing tools were useful in a Dutch health care setting and to modify these tools, if necessary. Furthermore, the reasons for inappropriate hospital stay had to be identified and possible solutions for these problems had to be assessed.

\section{Concepts of appropriateness}

Appropriateness in health care is a difficult and sensitive issue. This is illustrated by various related terms used in the literature, such as: 'inappropriate', 'inactive', 'unnecessary' or 'redundant'. 20 Also, literature indicates that inappropriateness can vary in time, place and with the instrument of measurement, depending on the setting of the health care, the patients involved and the way of measuring inappropriateness. "In addition, the assessment of the 'necessity of care' is difficult due to possible gaps in the evidence supporting treatment strategies and the evolving medical knowledge and technologies. ${ }^{22}$ Therefore, within the aims of this thesis, some boundaries were drawn. Firstly, the selected tool to asses appropriateness of hospital stay was the Appropriateness Evaluation Protocol (AEP) ${ }^{2-21}$ (chapter 4). The AEP is a widely used tool to assess the appropriateness of the patients' hospital admission or stay and consists of diagnosis-independent and care-related criteria. Secondly, only the appropriateness of the setting of the provided care was assessed. Neither the appropriateness of the provided care, nor the quality of this care, nor the patient's satisfaction have been studied within this context. Here, the basic assumption was that the provided care was medically appropriate and up to standard. ${ }^{25}$ Thirdly, only the appropriateness of the patient's stay in a hospital at specific index days was assessed, not the appropriateness of the patient's admissions to the hospital. For this purpose, appropriate hospital stay was defined as: "inpatient stay, requiring continuous and active medical, nursing or paramedical treatment, which under existing legislation can not be provided through extramural care, day care or outpatient care".

In summary, only issues concerning the appropriate setting of health care (chapter 2) have been considered, focusing on efficiency and timeliness of care. ${ }^{16}$ Hence, only "overuse" (chapter 2) of hospital facilities has been studied. It should be emphasized that if a day is deemed inappropriate by such a procedure, it does not indicate that the care itself, provided during that day, is redundant for the patient, but rather that this care could have been delivered by a facility that is less specialized than a hospital (e.g. a nursing home, hostel or home care). 
In this thesis, we especially focused on factors and processes affecting appropriateness of hospital stay, that are under the control of the hospital. These restrictions align with quality from a managerial perspective: using resources efficiently to give patients the care they need, without waste, errors or delay and within local policy and legal regulations (chapter 2). The first reason to focus on the appropriateness of hospital stay was that by avoiding or reducing inappropriateness extra capacity could become available and waiting times and waiting lists could be reduced. Secondly, if care provided in an inappropriate hospital setting could be transferred to an appropriate and less costly setting outside of the hospital, the costs of health care in general might be reduced.

\section{Research questions}

In this thesis, the central problem is defined as follows: 'Is the patient's stay in an acute care hospital appropriate and how can it be measured in a valid and reliable way?'. Therefore, several questions had to be answered such as:

1. 'What is appropriate hospital stay and which factors can affect it?'

2. 'How can we assess inappropriate hospital stay (IHS)?'

3. 'What kind of measurement instrument do we need?'

4. 'Can IHS be identified within specific processes or patients?'

5. 'Is IHS predictable?'

6. 'Which interventions are needed to reduce IHS?'

To provide an answer to these research questions several studies were set up in the University Hospital of Maastricht. The results of these studies are presented in the different chapters of this thesis.

\section{Organization of this thesis}

- Chapter 2 describes several concepts of appropriateness in order to provide an answer to the question: 'What is appropriate hospital stay?'. Special attention is given to the difference in the appropriateness of the setting versus the appropriateness of the provided care itself. In this chapter also the relationship of appropriateness with quality of care and quality costs is discussed. Several tools for quality assurance and utilization review are being compared.

- Chapter 3 gives insight in the recent trends in the Dutch health care and the factors related to the length of hospital stay. These factors can be divided in 'supply factors' and 'demand factors'. The correlation between these factors can indicate that 'A built bed is a filled bed' ${ }^{20}$, illustrating that if a service is offered or available, this service will be used.

- Chapter 4 consists of a literature review concerning the AEP and shows the first results of a pilot study of an instrument to identify and to measure IHS in the Dutch health care setting. Here, the study focused on the validity, reliability and applicability of the tool to assess the appropriateness of the hospital stay as well as the reasons for inappropriate hospital stay. Although this chapter was written as a preliminary study on inappropriate hospital stay by a different author, it contributes highly in understanding different ways of measuring IHS. This chapter is no part of any other thesis. 
- Chapter 5 focuses on further development and fine-tuning of the measurement instrument, called the Dutch Appropriateness Evaluation Protocol (DAEP). Emphasis was put on the validity, reliability and applicability of the DAEP. This instrument was used to assess the stay of patients admitted for the medical departments of Internal Medicine, General Surgery, Gynecology, Obstetrics, Neurology and Neurosurgery. This chapter also gives an overview of the results of the assessment of IHS and the related reasons for inappropriate stay at the University Hospital of Maastricht (azM).

- Chapter 6 discusses the need for extended day care (EDC) in a hospital setting. EDC patients are patients who undergo surgery and need hospital care longer than provided in the day care situation (i.e. after usual business hours), but less than 24 hours. This chapter assesses the appropriate length of EDC by means of the DAEP and a possible transfer to day care. It discusses the implementation of new policies on admission to and discharge from hospital. It also discusses whether the use of improved operation room scheduling or patient logistics could enable the transfer of EDC patients to a day care setting.

- Chapter 7 assesses the possibilities of detecting IHS within specific patient groups. Currently, treatment of deep venous thrombosis is usually provided in an ambulatory setting. The objective was to evaluate whether the care that was previously provided in a hospital setting was detected as inappropriate according to the DAEP. If so, this instrument could potentially be used to identify patient groups that could be treated in alternative settings.

- Chapter 8 presents the results of a study on possible predictors of IHS. Therefore, the hospital stay of surgical patients has been assessed. Also the average delay in hospital procedures and the causes of these delays were assessed. Patient characteristics were analyzed in order to predict possible IHS and to provide possible solutions to these problems. For this purpose a decision tree was designed to unambiguously classify the subsequent reasons of IHS.

- Chapter 9 shows the impact of several improvement actions in order to optimalize patient logistics and to decrease the delays in therapy or diagnostics. The objective was to decrease IHS related to delays in diagnostic procedures and hospital discharge among the patients admitted to the department of Internal Medicine. The overall results of these interventions and their specific effect are discussed.

- Chapter 10 assesses data on the appropriateness of hospital stay in obstetric patients and the effect of altered discharge procedures on the appropriateness of hospital stay and the length of this stay. The specific aims of this study were to evaluate the impact of alternative discharge procedures on IHS and to reduce it for mothers of newly born children.

- Chapter 11 consists of a general discussion about concepts and measurement of appropriateness. Extra attention is paid to some methodological issues. Furthermore, the lessons learned in the several studies are evaluated. Additionally, possible improvement strategies are discussed. 


\section{References}

1. Chassin M. Is health care ready for six-sigma quality? Milbank Q 1998; 76: 565-91.

2. Borst-Eilers E, Priority setting in the Netherlands. Second international conference on priorities in health care. Oct 9 th, 1998, London.

3. Drummond M, O'Brien B, Stoddard G, Torrance G. Methods for the economic evaluation of health care programs. $2^{\text {nd }}$ ed. Oxford: Oxford University Press, 1997.

4. Relman A. Assessment and accountability: the third revolution in medical care $\mathrm{N}$ Eng J Med 1988; 3 19: 1220-2.

5. Quaethoven P. Hospital performance and accountability. Paper, $8^{\text {th }}$ European Forum on Quality Improvement in Health Care. May $15^{\text {th }} 2003$, Bergen.

6. Joint Commission on Accreditation of Healthcare Organizations, Performance indicators, 2001, Oakbrook Terrace. Available from: URL http:/www.jcaho.org

7. Restuccia J, Holloway D. Barriers to appropriate utilization of an acute facility. Med Care 1976; 14:559-73.

8. Lavis J, Anderson G. Appropriateness in health care delivery: definitions, measurement and policy implications. Can Med Assoc J 1996; 154: 321-8.

9. Parsons, T. The Social System. Int Enc of Soc Sci 1968; 7: 429-41

10. Institute for Healthcare Improvement. Idealized design of clinical office practice, 1999, Boston. Available from: URL http://www.ihi.org

11. Grol R, Wensing M. Implementation; Effective change in patient care (in Dutch) Elsevier, 2001, Maarsssen, the Netherlands.

12. Bodenheimer T. The American health care system. The movement for improved quality in health care. N Eng J Med. 1999; 340: 488-92

13. Restuccia J. Holloway D. Barriers to appropriate utilization of an acute facility. Med Care 1976; 14:559-73.

14. Grol R. Beliefs and evidence in changing clinical practice. BMJ 1997; 315:418-21

15. Grol R, Grimshaw J. Evidence-based implementation of evidence-based medicine. J Qual Improv 1999; 25: 503-13.

16. Institute of Medicine. Crossing the Quality Chasm: A. New Health System for the 21 st Century. National Academy Press. 2000

17. Statistics Netherlands: Statline, the statistical database on the Netherlands cited $2001 \mathrm{Jul}$ 18. Available from: URL http:/argon2.cbs.nl/statweb/index_ENG.stm.

18. Brook R. Appropriateness: the next frontier. BMJ 1994; 308:218-9.

19. Schut M. Appropriate use of hospital beds. A study of the possibilities of the Appropriateness Evaluation Protocol (in Dutch). Maastricht University, 1994.

20. NuS Management Executive. What do we mean by appropriate health care? Quality in Health Care $1993 ; 2: 117-123$.

21. Restuccia J. The Evolution of Hospital utilization Review Methods in the United States. Int J Qual Health Care 1995; 7: 253-260.

22. Phelps C. The methodological foundations of studies of the appropriateness of medical care. N Engl J Med. 1993; 329: 1241-5.

23. Gertman P. Restuccia J. The appropriateness evaluation protocol: a technique for assessing unnecessary days of hospital care. Med Care 1981; 19:855-71.

24. Restuccia J. The Reviewers' Mamual. In: The AEP Fina! Report. Springfield, VA: Nat. Techn. Inf. Serv.. 1986, Publication No. PB87-112041.

25. Kahn K, Kosecoff J, Chassin M, et al. Measuring the clinical appropriateness of the use of a procedure. Can we do it? Med Care. 1988;26:415-22.

26. van Doorslaer E. van Vliet R. A built bed is a filled bed: an empirical recxamination. Soc Sci Med 1989: 28:155-64. 

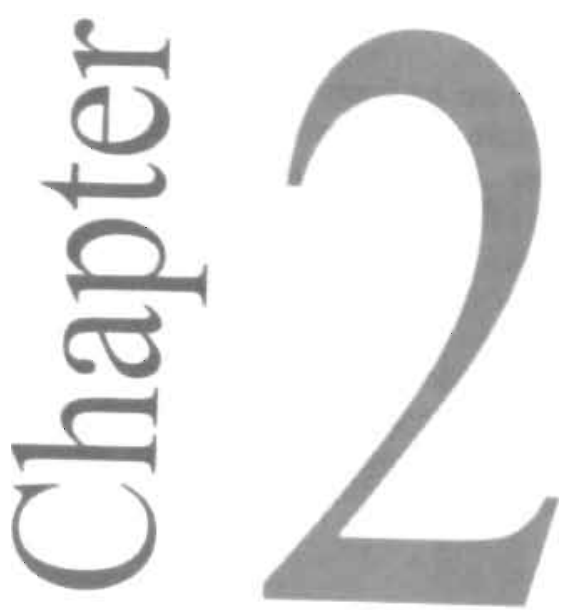

\section{The jumble of appropriateness}




\section{Introduction}

In health care, hospitals are under increasing pressure to improve the efficiency of internal operations, without sacrificing quality of care.. Care has to be effective and efficient and must have the highest possible quality. Health care is a set of complex systems with the patient, as a key-factor. So the system should be organized from his informed point of view: "to receive the required -and needed-help at the right time". This does not only benefit the patient, but also the society. To ensure that the right care is provided at the right time in the right place, accountability is essential. A central feature of accountability is ensuring that appropriate services are provided in appropriate settings. However, where does appropriateness end and where does inappropriateness start? So, in order to assess the appropriateness some concepts have to be clarified.

\section{What is appropriate hospital stay?}

Appropriateness seems to be an answer for resource allocation in the numerous diagnostic and therapeutic procedures that are currently available. ${ }^{1 \cdot 2}$ Appropriateness is often treated as an isolated concept. However, there are two distinct. types of appropriateness: appropriateness of care and the appropriateness of the setting in which that care is provided.

The appropriateness of care is related to effectiveness and means that the expected benefits to a patient outweigh the expected harms to that patient. Hereby the procedure is labeled as "necessary" if it must -for reasons of ethics and medicall necessity- be offered to the patient. In this context is assumed that a qualified person provides the procedure in the setting of a particular health care system. If a procedure is provided for a patient for whom it is "unnecessary" it is called inappropriate and defined as an "overuse" of the procedure. If the procedure is not offered for a patient for whom it is necessary, it is termed "under-use" of the procedure.

The appropriateness of the setting in which care is provided is related to costeffectiveness. This type of appropriateness is determined by the match of the clinical characteristics and requirement of services for care of a patient with the setting in which this care is provided. Setting is a proxy measure of the resources used to provide care. Just as effective care can be provided in a manner that is not cost-effective, appropriate services can be provided in inappropriate settings. When appropriateness of setting is considered, it is assumed that the services are appropriate and are provided in a technically correct way. An inappropriate setting (i.e. inappropriate hospital use) can be defined as use that is not suitable to the patient's medical needs. ${ }^{3}$ There are two types of inappropriate use. Over-use is care that is of no benefit to the patient or care that could be provided in a less costly setting. Underuse is care that is not sufficient to meet the patient's medical needs. The relationship between the patient's need and the services provided is highlighted in figure 1. 'Both under-and overuse have implications for the cost and quality of care. Underuse is clearly related to quality of care: the patient receives insufficient treatment, with all possible negative consequences. Overuse has a clear impact on health care costs: unnecessary use increases costs. And it has also important quality-of-care implications: the longer the stay, the higher the risk for hospital-acquired complications (infections, etc.). 


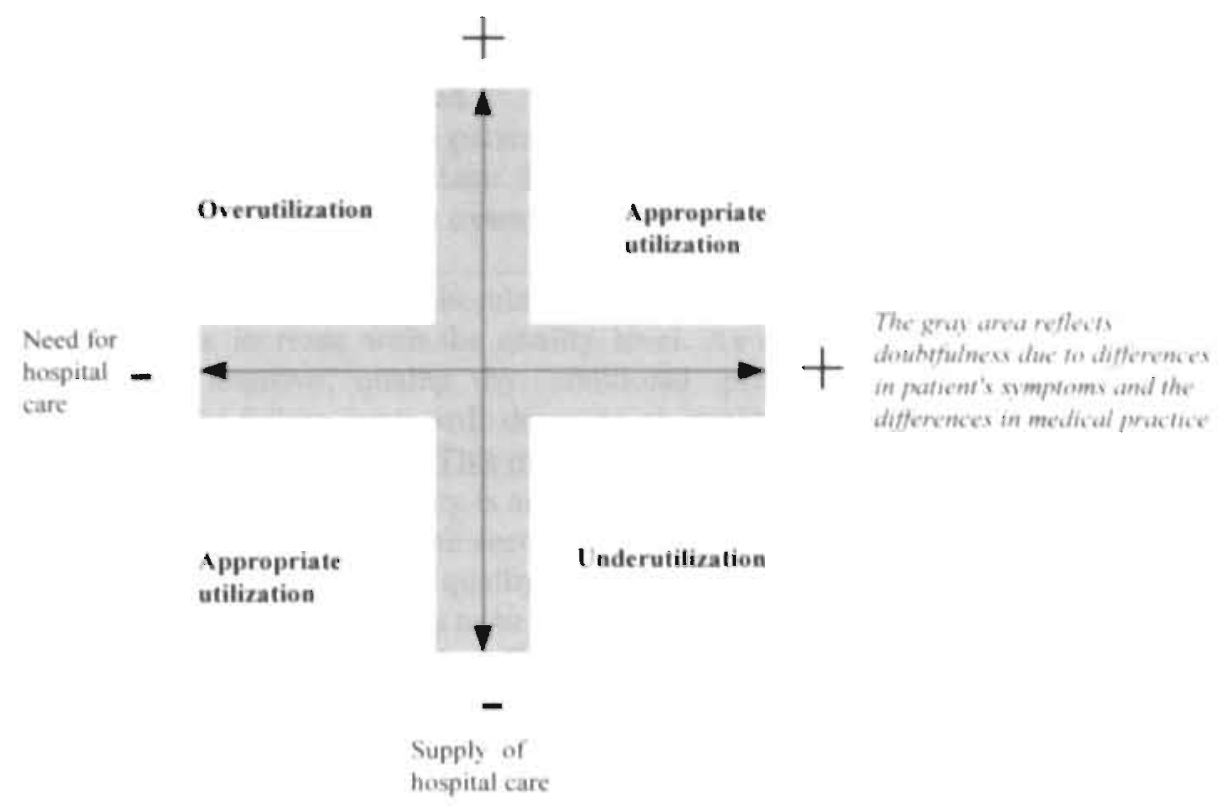

\section{What is a good health care quality?}

in heaith care different professions and depariments have different understandings aboul what quality means. Without a simple definition of quality it is difficult to define objectives for quality improvement efforts. The definition of quality should be in accordance with professional values, convey the patient's focus and bring in concepts of gquality management, reducing waste and increasing efficiency. A definition of guality which many professionals have found to work best is the three-dimensional definition of patient, professional and management quality: ${ }^{8}$

1. Patient quality is what patients say they want;

2. Professional quality is what professionals think patients need (outcome and process);

3. Management quality is using the fewest resources to give patients what they want and need, without waste, errors or delay and within policy and legal regulations.

Good health care quality should combine all three dimensions at the same time. So, if quality activities are performed in the right way, no trade-off between increasing patient satisfaction, improving professional outcomes and reducing costs will occur.

\section{What are quality costs?}

Since inappropriate use of health care services can be seen as a loss or waste of quality, it is important to realize that there are different types of quality costs. Several concepts have been developed to explain the relationship between costs and quality. These concepts provide a common frame for both quality managers and financial managers. Quality costs are the costs due to, or to prevent deviations of quality standards. There are four types of quality costs: prevention, appraisal, and internal and external failure costs (fig. 2). ${ }^{9-10}$ Several studies in the profit sector point out that quality costs are an important part of business costs. Up to $25 \%$ of all business costs in the profit sector and up to $40 \%$ in the non-profit sector are possible quality costs. For health care 
organizations, quality costs are estimated to be: $25-30 \%$. "To split up quality costs in prevention, appraisal and failure costs is a bit high-handed, but still it is useful to translate possible causes of quality related problems into financial terms.

Fig. 2: Classification of quality costs

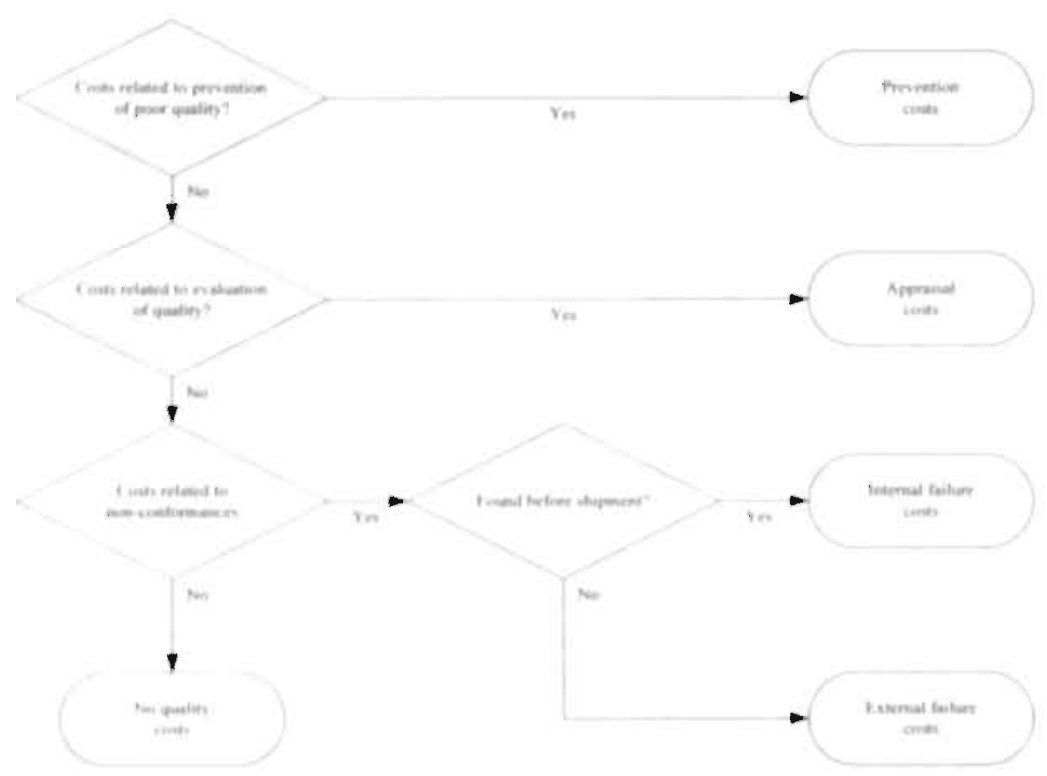

Prevention costs

I'hese are costs made to prevent errors or non-conformances in order to avoid quality problems and to keep appraisal and failure costs to a minimum. Examples include instruction, training, process control and quality planning.

Appraisal costs

These are the costs refated to the implementation of systems to monitor the quality of products and services, i.e. the degree of conformance to quality requirements. Expenses made to perform audits are an example of this type of costs.

\section{Failure costs}

These are the expenses that have to be made to correct errors or non-conformances. These costs would disappear if no errors or non-conformances existed in the products or services. In industry, a difference is made between internal and external failure costs for errors found before or after the shipment to the customer. In health care these boundaries cannot explicitly be made since in health care production and consumption occur simultaneously. If (any step in) the process of providing care is delayed, internal failure costs occur. E.g. if patients charts or test results are not available at the moment of the patient appointment, this causes waste of time for both the patient and the organization. In health care some internal failure costs are unavoidable. Even if the process is run perfectly. complications in the patient's condition may occur. Internal failure costs are not always caused by the organization. but can also be caused by the 
patient, e.g. if the patient does not keep his appointment. Internal failure costs can be identified and quantified by process analysis. Through process analysis, frequent problems inducing additional costs can be identified and tackled. Also variation in clinical practice can be detected. ${ }^{12-13}$ External failure costs increase expenses after the process is completed. E.g. if a patient is dissatisfied with (the results of) his treatment, he might file a complaint or claim. Dealing with this complaint or claim will generate additional costs. So, claims are examples of external failure costs.

The amount of quality costs is related to the achieved quality level. Prevention and appraisal costs increase with the quality level. As quality increases, it will be more difficult to improve quality by additional prevention or evaluation actions. Simultaneously failure costs will decrease as quality improves. So, the total quality costs show a U-shaped curve. This means that quality costs will be high in case of poor quality but also if perfect quality is aimed for. In the first situation high quality costs are due to high failure costs, in the second situation these are due to high prevention and appraisal costs. The concept 'quality costs' and the relation with the level of quality shows that quality efforts have to be dosed properly. An organization with high failure costs should question itself if the efforts to handle failures could not be used in favor of more prevention or appraisal activities. These activities prevent, rather than solve problems. On the other hand, organizations with high prevention and appraisal costs and almost no quality problems must realize that they can save money (time, materials and efforts) if they allow slight and controlled quality problems. Of course, this issue depends on the vision and mission of the top management and the kind of activities deployed. The U-shaped relation between quality costs and quality indicates that quality costs do not decrease by inferior or perfect quality (fig. 3). Especially in health care, perfect quality can only be achieved by extreme high prevention and appraisal costs.

Fig. 3: Optimum quality costs model

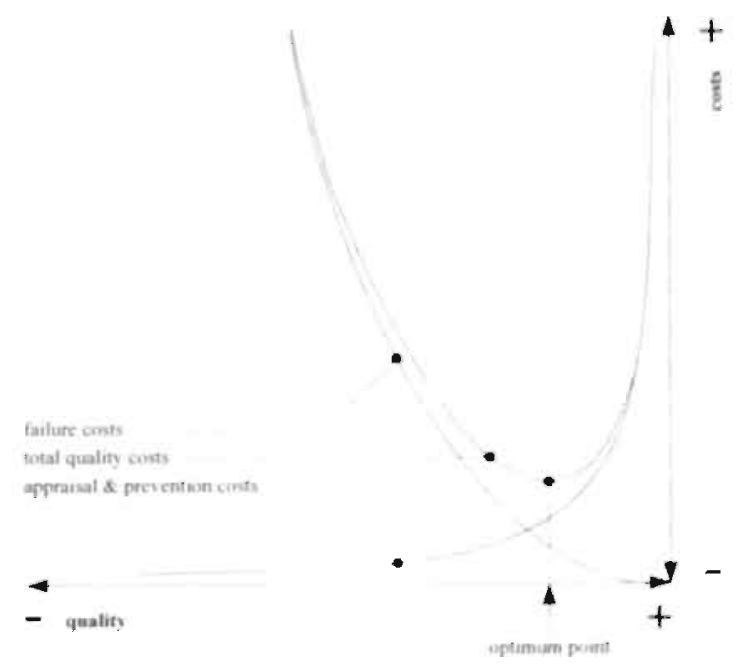




\section{Appropriateness versus costs and quality}

As mentioned before, appropriateness is closely related to costs and quality. The past years, two other concepts are more and more emphasized; cost containment and quality assurance. ${ }^{14}$ If these two concepts are projected on a coordinate system, four possible situations occur. Figure 4 shows these four situations as four quadrants, formed by costs. and quality. These four quadrants are combinations of quality improvement or quality loss and a decrease or increase of costs. The intersection of costs and quality represents the situation at this moment.

Fig. 4: Costs vs. qualify

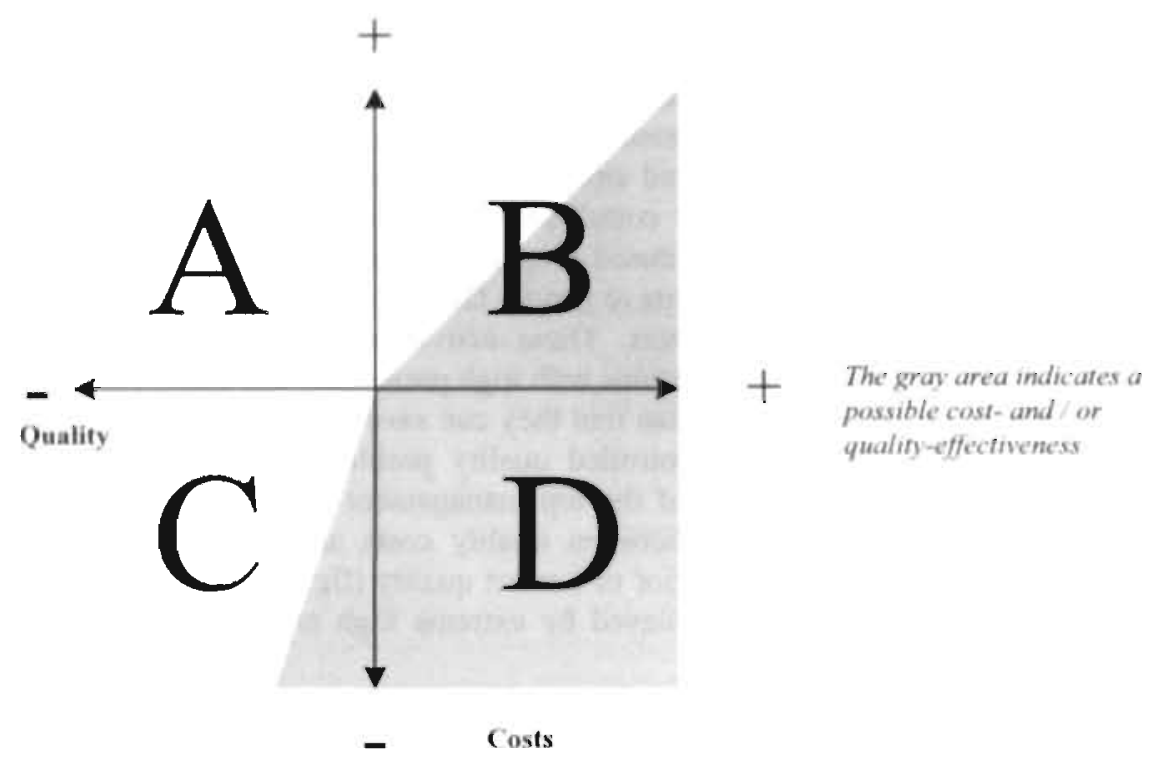

A shift to the right indicates quality improvement; a shift to the left indicates quality loss. Moving upwards will show an increase in costs, moving downwards means cost reduction. Quadrant D is most desirable; here quality improvement and cost containment meet together, reducing quality waste. This is what health care providers and their organizations aim for. Improving e.g. the scheduling of operation room (OR) procedures is an example of this quadrant. Improved scheduling provides a more efficient use of staff personnel and less overtime has to be compensated. The customers will be satisfied, since fewer procedures are delayed and staff personnel will show a higher degree of job satisfaction, since the workload is better predictable. Quadrant A shows an increase in costs and a loss of quality. This means quality waste and has to be avoided. ' E.g. unnecessary use or overuse of new (and expensive) antibiotics not only leads to an increase of costs, but, in the long run. also to lower quality of care (antibioticsresistance). A longer stay in the hospital not only generates more costs but also increases the risk of hospital-acquired infections (worse outcome). These interventions might harm the patients ${ }^{\prime}$ health. In quadrant B there is a quality improvement with a rise in costs. However, here the question remains if quality improvement can be justified by an increase in costs. So cost-benefits are required / indicated. In case of an upward shift: along the vertical axis (same quality and more costs) there is an inefficiency waste. Here 
experts indicate a potential saving of over $50 \%$. ${ }^{15}$ The opposite occurs in quadrant C (cost reduction and quality loss), but here the situation in the gray triangle is the least unattractive, since there is large cost reduction with small quality loss (reduction of efficiency waste). This figure shows similarity with studies on cost-effectiveness, indicating that people are willing to accept quality loss at an acceptable price. ${ }^{10}$. Yet, the question remains: which degree of cost reduction does not yield an unacceptable loss of quality! In other words, which situation is appropriate?

\section{The assessment of appropriateness in health care}

Utilization review ${ }^{17}$ and quality assurance are the most common ways to assess the appropriateness in health care. Quality assurance focuses on the effectiveness with which hospital services are provided (the benefit per unit). Often with less emphasis on the costs of the services provided than is true with utilization review. ${ }^{18}$ Quality assurance does not only review of the structure -as in utilization review- but also reviews the process and outcome of care, the side-effects of a treatment, and at times. the interpersonal relationship between provider and patient. ${ }^{19}$ Utilization review is closely related to quality assurance and focuses on the efficiency with which care is provided (cost per unit). The purpose is to assess the efficiency of the health care process and the appropriateness of decision making related to the site of care, its frequency and its duration. ${ }^{20}$ It can be conducted either before admission, during the patient's stay, or after discharge by reviewing the patient's medical record through application of defined criteria and/or expert opinion. Both techniques rely on review of the individual medical record and both evaluate the relationship between the patient's need for (medical) services and the services received. Utilization review is an important component of utilization management, which is deliberate action to increase the efficiency or the effectiveness with which hospital services are provided. Utilization management can have several benefits; reducing costs through reducing unnecessary hospital use, maintaining quality of care by assuring that the hospital services are provided effectively, preserving access to care by reserving hospital resources for those who need inpatient care, and defining standards of care. Utilization review information can be used in several ways, especially when it is obtained with other routinely collected data. It can be concentrated on the type of patients most likely to experience inappropriate hospital stay. This information can identify the characteristics of a patient, physician, hospital service or hospital stay that are associated with higher levels of inappropriate use through calculating simple percentages. Information on the reasons for inappropriate stay can be used to design improvement actions. ${ }^{21}$ Services with high rates of inappropriate hospital stay and high patient volume are good candidates for focused review and corrective actions. One procedure to assess inefficiency in the use of acute care hospitals is the use of tools designed to assess the appropriateness of hospital admission and subsequent days spent in hospital by the retrospective or concurrent application of objective criteria. Three tools widely used for this procedure are the ISD criteria set (Intensity of service, Severity of illness, Discharge screens), ${ }^{22}$ the AEP (Appropriateness Evaluation Protocol) ${ }^{23}$ and the MCAP (Managed Care Appropriateness Protocol). ${ }^{24}$ 


\section{Conclusion}

These concepts offer some useful philosophies for general and quality management of an organization. The concepts of 'quality costs' are developed from the view of efficiency improvement. The aim is not a better quality at any price, but this better quality is put against the means that are needed to reach the goals. The link between the concepts of costs and quality shows that the aim of quality improvement is not always the best option. Quality improvement is limited by scientific and technologic possibilities. The investment in quality improvement can implicate that there are less resources for the routine processes of an organization. In the end this will lead to other quality problems. These concepts provide possibilities for joint discussions. When in a short period quality improvement has to be achieved by the use of more resources, the top management must be convinced of the advantages of this investment. These concepts become more understandable if they are related to the classic efficiency concepts. However, the translation of these concepts into financial terms is challenging. In spite of this challenge, these concepts are useful to start the communication between the several departments of an organization. This improvement in communication is already a quality improvement. These instruments also provide a better insight in the costs of poor quality by determining the cause of the problem(s) through process analysis. Finally these concepts provide an instrument to prioritize projects on quality improvement; attention can be paid to avoidance and influence of (quality) costs.

The 'Iength of stay' is still used as a parameter to assess the efficiency of the provided care. In the past we have seen a constant decrease in the length of stay. Reducing the time that patients spend in hospital is seen as one way to control health care costs. Yet, more and more the suggestion is raised that care setting or 'level of care' (the type and intensity of skilled services and setting in which they are provided) should replace 'length of stay" as a criterion for efficient hospital use. So, 'appropriate hospital stay' would mean that the patient receives a level of care available only in the hospital. ${ }^{25}$ Yet. insight in factors related to the patient's stay in the hospital is essential to assess the appropriateness of this stay. 


\section{References}

1. Buetow S. Sibbald B, Cantrill J, Halliwell S. Appropriateness in health care: application to prescribing. Soc Sci Med. 1997; 45:261-71.

2. Appropriateness as a tool for quality assurance in health policy. Paper, 66th European Health Policy Forum, March 25-26, 1999, Louvain, Belgium.

3. Payne S. Identifying and managing inappropriate hospital utilization: a policy synthesis. Health Serv Res 1987; 22: 709-69.

4. Lavis J. Anderson G. Appropriateness in health care delivery: definitions, measurement and policy implications. Can Med Assoc J 1996; 154(3): 321-8.

5. Restuccia J, Holloway D. Barriers to appropriate utilization of an acute facility. Med Care 1976; 14:559-73.

6. Zhan C, Miller M. Excess length of stay, charges, and mortality attributable to medical injuries during hospitalization. JAMA. 2003; 290:1868-74.

7. Leu H, Kaiser D, Mori M, Woolson R, Wenzel R. Hospital-acquired pneumonia. Attributable mortality and morbidity. Am J. Epidemiol. 1989; 129:1258-67.

8. Ovretveit J. The Norwegian approach to integrated quality. Health Care Review, $2000 ; 2:$ I. 28 Jan.

9. Juran J, Gryna F. Company wide assessment of quality: In: Quality planning and analysis. 3th ed. McGraw-Hill Inc. New York, 1993.

10. Wortman B. The Quality Engineer Primer. Quality Council of Indiana, Sixth Edition Update, Aug 6, 1999.

11. Peters T. Thriving on chaos, 1987. New York, Alfred A Knopf.

12. Wennberg J, Gittlesohn A. Variation in medical care among small areas. Scient American, 1982; 246.4:120-34.

13. Wennberg J, Cooper M. The Darthmouth atlas of health care in the United States. American Hospital Publishing Co., 1998, Chicago.

14. Kesteloot K. Quality and costs: methods and concepts. (in Dutch) Acta Hospitalia $2000 ; 1: 7-18$.

15. James B. Qualin controls cost. Available from URL http://www.ihc.com/ documents/8/TrackCST.pdf.

16. O’ Brien J. Gertsen K. Willan A, Faulkner L. Is there a kink in consumers 'hreshold value for cost-effectiveness in health care? Health Econ 2002; 11: 175-80.

17. Donabedian A. Explorations in quality assessment and monitoring: the methods and findings of quality assessment and monitoring: an illustrated analysis: Ann Harbor, MI: Health Administration Press, 1985(2).

18. Caterinicchio R. Integrated concurrent utilization quality review: I Qual Assur. 1987:9:14-5.

19. Donabedian A. Aspects of medical care administration: specifying requirements for health care. Cambridge, MA: Harvard University Press, 1973.

20. Donabedian A. Explorations in quality assessment and monitoring: the criteria and standards of quality (volume II). Ann Harbor, MI: Health Administration Press, 1982.

21. Payne et al. Using utilization review information to improve hospital efficiency. Hospital \& Health Serv Adm 1991;36:473-90

22. The InterQual review system. Marlborough (MA): InterQual Products Group, 1996.

23. Gertman P, Restuccia J. The appropriateness evaluation protocol: a technique for assessing unnecessary days of hospital care. Med Care 1981; 19:855-71. 
24. The Managed Care Appropriateness Protocol. Wellesley (MA): Oak group, 1996.

25. Goldberg G. Holloway D. Emphasizing 'level of care' over 'length of stay' in hospital utilization review. Med Care 1975; 13: 474-85. 

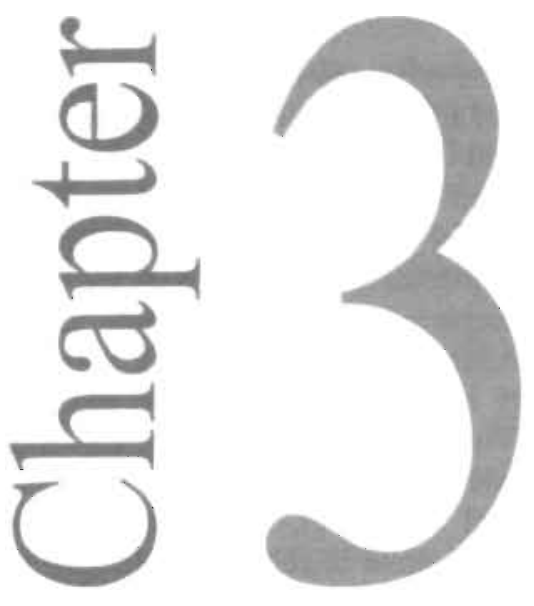

Factors affecting hospital stay 


\section{Factors related to hospital stay}

The patient's clinical condition is a very important factor related to hospital stay, but not the only one. Several other factors affect the length and appropriateness of hospital stay. This stay has been reduced during the past decades, due to cost containment actions and the search for a higher efficiency. Nevertheless, it is still important to explore reasons, other than the patient's condition, that are responsible for the patient's hospitalization. Literature indicates that hospital stay might be related to characteristics of the care provider, ${ }^{1-5}$ i.e. the individual practice style of a clinician or the available beds in an institution. These are described as supply factors. Alternatively, hospital stay may be related to the patients' needs - for instance: severity of disease, comorbidity, time of recovery, or costs to the patient of a longer stay. ${ }^{6}$ These can be described as demand factors (see table I). It is important to distinguish between demand factors (i.e. the need for care) and supply factors (i.e. the provision of the care). If supply factors predominate, methods of practice and managerial constraints to reduce the length of hospital stay might be appropriate. Supply factors are closely related to the setting of care and its appropriateness. Since this thesis focuses on the setting of care (supply factors), the demand factors are only discussed briefly.

Table I: Reasons of variation in length of stay

\section{Supply factors}

- Individual practice style

- Bed supply and availability of primary, community, or convalescent care

- Discharge policies, level of illness at which hospital care is considered desirable

Demand factors.

- Socio-economic status

- Disease severity

- Comorhidity

\section{Supply factors}

- Individual practice style

Decision-making behavior of clinicians can be a determinant of length of stay (e.g. being too cautious in the management of a patient). ${ }^{7}$ Aiso, seniority and experience of the medical staff affects length of stay. ${ }^{8}$ One study showed that length of hospital stay declined by 0.43 days as junior doctors became more experienced over the academic year. "In the past, efforts have been made to change clinicians' behavior, particularly at the planning of a patient's discharge from the hospital. But, although studies assessing different methods of planning discharge (e.g. clinical pathways, continuous feedback) found changes, it was difficult to identify the exact nature or cause of the differences found in the length of stay. ${ }^{10}$

- Bed supply

The effect of availability of beds on. length of stay has been investigated in several studies. Their results suggest that the supply of beds is the factor that is most closely associated with length of stay. ${ }^{11-13}$ The larger the amount of beds available, the longer the hospital stay became. A large study on regional variations on length of stay suggested that the strongest determinant was bed supply and the results were summarized as: "A built bed is a filled bed." "This indicates that if health care services of facilities are offered or are available, they will be used. So, the availability of too many health care facilities carries a great danger of overuse. 
- Hospital discharge policies

Length of stay is also influenced by hospital discharge policies. ${ }^{14-17}$ Some studies ${ }^{12-}$ 13 indicate that patients who have been longer on waiting lists were discharged earlier because of an increased pressure on the available hospital beds. Hospitals, able to send patients to convalescent facilities or less specialized hospitals, did transfer these patients in an earlier stage. This smooth transfer from acute wards either to other hospitals or to convalescent facilities could be a reason for shorter stay. ${ }^{9.10,12}$ 18-19

\section{Demand factors}

The patient's condition should be the main factor determining the length of stay. Beside this, the patient's preferences should be taken into consideration. ${ }^{3,20-22}$ Often medical decisions are made, based only on the preferences of the physician. ${ }^{21.27}$ Therefore, the patient should be supported in shared decision-making. ${ }^{28.29}$ However, results of several large studies show that the patients' individual needs do not seem to be related to the length of stay. ${ }^{6,9-13,18-10,30-36}$ Complications can act as a confounding variable. A longer stay may be more likely to result in complications such as infection acquired in hospital. However, complications may also increase length of stay, as the patient's condition obstructs hospitall discharge at the scheduled day. There are complex explanations for the considerable variations that exist in length of stay in hospital.

\section{Recent evolutions in hospital use}

In all industrialized countries similar evolutions in health care can be detected over the past decade. These evolutions can be related to cost containment actions. The number of acute care beds decreased by $10-30 \%$ (fig. 1) and the length of hospital stay decreased on average by 1 - 2 days (fig. 2). Yet, health care costs did not decrease (fig. 3). Figures 1 to 3 are based on data from the Organization for Economic Co-operation and Development ${ }^{40}$ and show the differences in evolutions in health care in different countries. But these comparisons have to be interpreted cautiously since different countries have different methods of recording and collecting data and different approaches to quality control. It is remarkable that hospital stay appears to depend not entirely on the patient's needs. Often the preferences of the health care provider determine the lype of care a patient receives (in full consent). ${ }^{23,28-29,41-44}$ The medical professional is often scen as the expert and as the patient may be overwhelmed by the news of his (bad) health situation, or by the lack of medical knowledge, the suggestions of the health care provider are accepted. Also, the organization of a health care system seems to be an important determining factor for the length of hospital stay. As an example: in comparison to Belgium, patients in the Netherlands stay on average two additional days in hospital.

\section{Recent evolutions in Dutch hospital use}

Figure 4 shows the evolution of hospital use in the Netherlands, with the level observed in 1990 as point of reference. ${ }^{45}$ Although the number of hospital beds decreased by approximately $25 \%$, the number of admissions to an acute care hospital stayed the same. In spite of this, health care costs increased, even as expressed as a percentage of the gross national product over the past 10 years. A part of this increase can be explained by the fact that day care admissions doubled in these years, generating their own costs. Additionally, it is likely that the patients who stay in the hospital need relatively more care, resulting in actual hospital days that are more expensive. 
Fig. 1: Evolution in acule care beds (per 1,000 inh.)

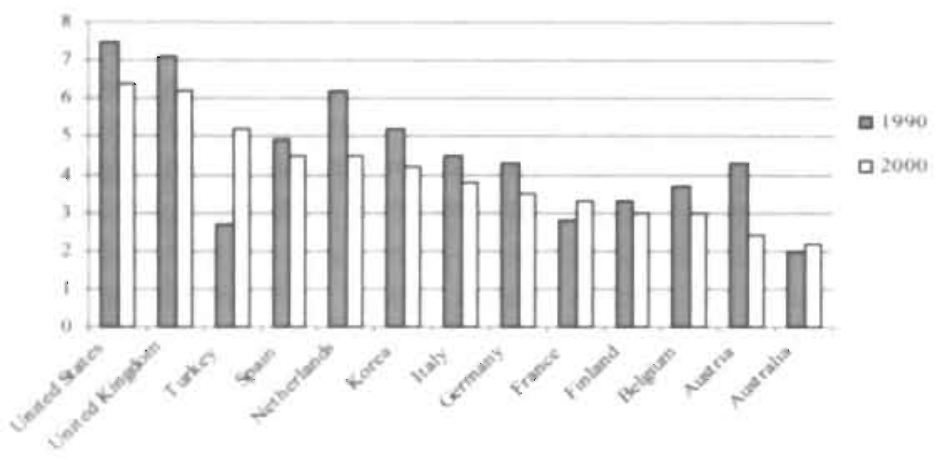

Fig. 2: Evolution in length of hospital stay (in days)

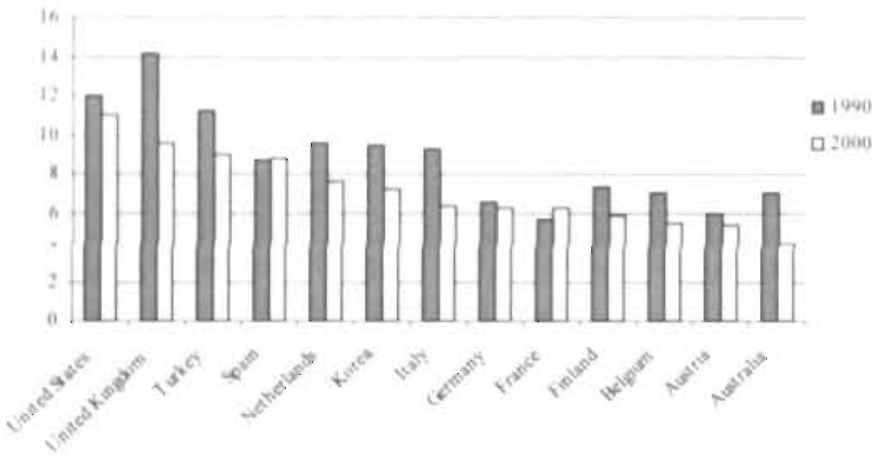

Fig. 3: Evolution in health care costs (in \% of GNP)

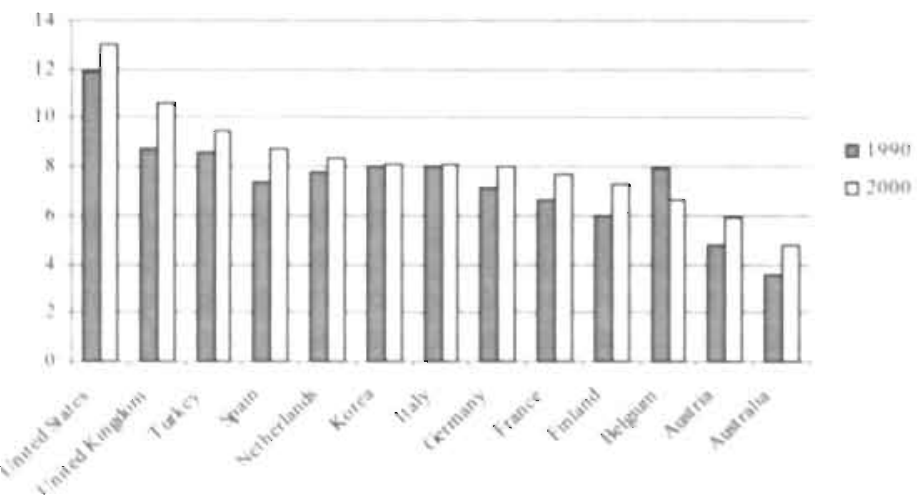




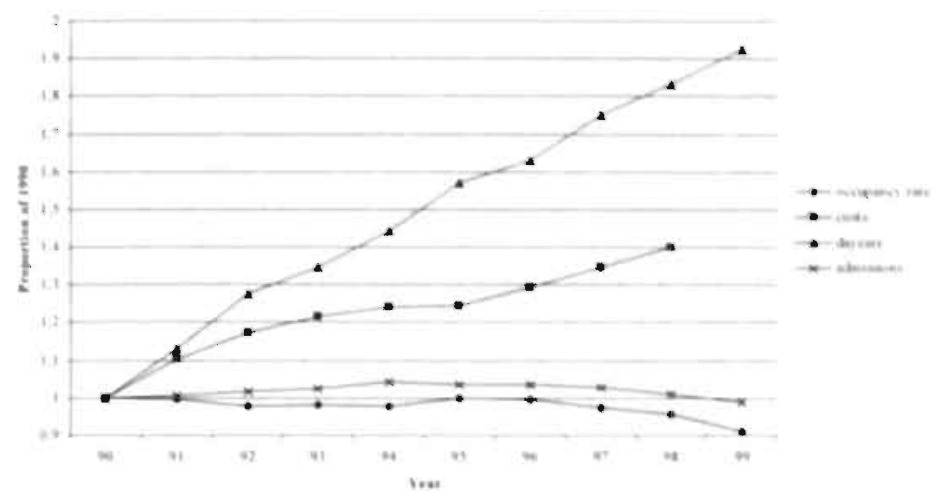

\section{Conclusion}

Supply factors are shown to have influence on how long a patient stays in a hospital. Bed supply seems to have the strongest associations with hospital stay. ${ }^{10-13}$ The activities of the medical staff may have some effect, but this influence is limited. ${ }^{4-6,9-10}$ Investigation of a variety of demand factors seems to suggest that patients may have the least influence of all. Therefore a shared decision-making between patient and physician may be needed. ${ }^{23},{ }^{28-29}$ Publications on the effect of length of stay on clinical outcome are few and might become rapidly outdated as the stays become shorter over time. Nevertheless, the literature suggests that there is a relation in patient outcome and length of hospital stay. ${ }^{46-51}$ Patients might even experience a worse outcome from a shorter stay or vice versa. For example, studies of wound breaking strength in surgical cases might suggest that there is a theoretical minimum period during which bed rest might be desirable, as hospital care may also protect patients from onerous household chores or adverse home circumstances. But complications such as the development of thromboembolic disease ${ }^{52}$ or the development of hospital-acquired infection ${ }^{51}$ are associated with longer stay. Therefore physician have to reflect. constantly on the length of stay, since there are complications related to short and long stay.

In summary, if there are facilities, they will be used. "This may lead to overuse. But, this overuse may lead to misuse as it may harm the patient. Therefore, the patient should only stay in the hospital as long (i.e. short) as medically required. This means that health care services also have to be available outside a hospital setting. And last, but not least, both relatives and patients may gain a great deal of pleasure from early, but medically appropriate discharge, since the patient can recover in a familiar surrounding and relatives do not have the inconveniences of traveling to the hospital. But it should be realized that volunteer aid could be a burden in the long run. 


\section{References}

1. Clarke A, Rosen R. Length of stay. How short should hospital care be? Eur J Public Health 2001; 11:166-70

2. Clarke A. Length of hospital stay and its relationship to quality of care. Qual Saf Health Care 2002; 11:209-10

3. Wennberg J. Giving patients a bigger say in choosing treatment. Med Econ. 1993 Oct 11; 70: 138-42

4. Wennberg J, Caper P. Medical practice: why does it vary so much? Hospitals. 1985 Mar 1; 59: 88-90

5. Wennberg J, Gittelsohn A. Variations in medical care among small areas. Sci-Am. 1982 Apr; 246: 120-34.

6. Burns L, Wholey D. The effects of patient, hospital, and physician characteristics on length of stay and mortality. Med Care 1991; 29:251-71.

7. Angelillo I, Ricciardi G, Nante N, Boccia A. Bianco A, La Torre G, Vinci V, De Giusti M. Appropriateness of hospital utilisation in Italy. Publ Health 2000; 14: 9-14

8. McKeown R, Reininger B, Martin M, Hoppmann R. Shared decision-making: views of first-year residents and clinic patients. Acad Med. 2002; 77:438-45.

9. Rich E, Gifford G, Luxenberg M, Dowd B. The relationship of house staff experience to the cost and quality of inpatient care. JAMA 1990; 263: 953-7.

10. Marchette L, Holloman F. Length of stay, significant variables. J Nursing Admin $1986 ; 16: 12-9$.

11. van Doorslaer E, van Vliet R. A built bed is a filled bed: an empirical reexamination. Soc Sci Med 1989; 28:155-64.

12. Beech R, Withey C. Morris R. Understanding variations in lengths of stay between hospitals for fractured neck of femur patients and die potential consequences of reduced stay targets. J Public Health Med 1995; 17:77-84.

13. Robinson J, Luft H, McPhee S, Hunt S. Hospital competition and surgical length of sta: Journal of the American Hospital Association 1988; 259:696-700.

14. Dautzenberg P. Bremer R, Lomans, M Tanis J. Ontslagvertraging in het algemeen ziekenhuis. Een oriënterend onderzoek in 's Hertogenbosch. Med contact 1997; 42:1310-3

15. Braveman P. Kessel W. Egerter S, Richmond J. Early discharge and evidence-based practice. Good science and good judgement. JAMA 1997; 278:334-6

16. Houghton A et al. Does a dedicated discharge coordinator improve the quality of hospital discharge? Qual in Health care 1996; 5:89-96

17. Vlugt, van der M. et al. Vroeg ontslag na een hartinfarct. Hartbulletin 1996; 27:62-6

18. Roemer M. Bed supply and utilisation: a natural experiment. Journal of the American Hospital Association 1961; 35:34-42.

19. Griffiths M. Waters W, Acheson E. Sickness absence after inguinal herniorrhaph: J Epidemiol Community Health 1979; 33:121-6.

20. Ganz P. What outcomes matter to patients: a physician-researcher point of view: Med Care. 2002;40 Suppl:III :11-9.

21. Bowling A. Ebrahim S. Measuring patients' preferences for treatment and perceptions of risk. Qual Health Care. 2001: 10 Suppl 1:2-8,

22. Bruera E, Sweeney C. Calder K. Palmer L. Benisch-Tolley S. Patient preferences versus phsician perceptions of treatment decisions in cancer care. J Clin Onc 2001:19:2883-5.

23. Eraker S. Politser P. How decisions are reached: physician and patient. Annals of Internal Medicine 1982; 97:262-8 
24. Mandelblatt J, Berg C, Meropol N, Edge S, Gold K, Hwang Y, Hadley J, Measuring and predicting surgeons' practice styles for breast cancer treatment in older women. Med Care. 2001; 39:228-42.

25. Elwyn G, Edwards A, Wensing M, Hibbs R, Wilkinson C. Grol R. Shared decision making observed in clinical practice: visual displays of communication sequence and patterns.J Eval Clin Pract. 2001: 7:211-21.

26. McKinstry B. Do patients wish to be involved in decision making in the consultation? A cross sectional survey with video vignettes. BMJ. 2000; 321:867-71.

27. Frosch D, Kaplan R. Shared decision making in clinical medicine: past research and future directions. Am J Prev Med. 1999; 17:285-94.

28. Kasper J, Mulley A, Wennberg J. Developing shared decision-making programs to improve the quality of health care. Quality Review Bulletin 1992; 18: 183-90

29. Mulley A. Supporting the patient's role in decision-making. Journal of Occupational Medicine 1990; 12:1127-8

30. Morgan M, Beech R. Variations in lengths of stay and rates of day case surgery: implications for the efficiency of surgical management. J Epid Comm Health 1990: 44:90-105.

31. Robbins J, Donaldson L. Analysing stages of care in hospital stay for fractured neck of femur. Lancet 1984; 2:1028-9.

32. Wade D. Hewer R. Hospital admission for acute stroke: who, for how long, and to what effect? J Epidl Comm Health 1985; 39:347-52.

33. Johnson A. Dowd B, Morris N. Lurie N. Differences in inpatient resource use by type of health plan. Inquiry 1989: 26:388-98.

34. Stem R, Juhn P. Gertler P, Epstein A. A comparison of length of stay and costs for health maintenance organization and fee-for-service patienis. Arch Int Med 1989; 149:1185-8.

35. Bradbury R, Golee J, Stearns F. Comparing hospital length of stay in independent practice association HMOs and traditional insurance programs. Inquiry 1991; 28:87-93.

36. Chen E, Naylor C. Variation in hospital length of stay for acute myocardial infarction in Ontario Canada. Med Care 1994; 32:420-35.

37. Westert G, Lagoe R, Keskimaki I, Leyland A. Murphy M. An intermational study of hospital readmissions and related utilization in Europe and the USA. Health Policy. 2002; $61: 269-78$.

38. Jacobs P. Edbrooke D, Hibbert C, Fassbender K. Corcoran M. Descriptive patient data as an explanation for the variation in average daily costs in intensive care. Anaesthesia. 2001:56:643-7.

39. Ensberg M, Paletta M. Galecki A. Dacko C, Fries B. Identifying elderly patients for early discharge after hospitalization for hip fracture. J Gerontol. 1993; 48: 187-95.

40. Organisation for Economic Co-operation and Development, OECD IIEALTH DATA 2002 4th ed. available from URL: http:/www.oecd.org/statistics/

41. Kronick R, Goodman D, Wennberg J, Wagner E. The marketplace in heallh care reform. The demographic limitations of managed competition. $\mathrm{N}$ Engl J Med. 1993 Jan 14:328: 148-52.

42. Wennberg J. Which rate is right? N Engl J Med. I986 Jan 30; 314:310-1

43. Wennberg J, Caper P. Medical practice: why does it vary so much? Hospitals. 1985 Mar 1: 59: 88-90 
44. Wennberg J, Gittelsohn A. Variations in medical care among small areas. Sci-Am. 1982 Apr; 246: 120-34.

45. Statistics Netherlands: Statline, the statistical database on the Netherlands. Available from: URL http://argon2.cbs.nl/statweb/index ENG.stm.).

46. Becker P, McVey L,Saltz C, Feussner J, Cohen H. Hospital-acquired complications in a randomized controlled clinical trial of a geriatric consultation team.JAMA.1987; 257:2313-7

47. Kalish R, Daley J, Duncan C, Davis R, Coffman G, lezzoni L.Costs of potential complications of care for major surgery patients. Am J Med Qual.1995; 10: 48-54

48. Roos L, Stranc L, James R, Li J. Complications, comorbidities, and mortality: improving classification and prediction. Health-Serv-Res. 1997; 32: 229-38

49. Norton E, Garfinkel S, McQuay L, Heck D, Wright J, Dittus R, Lubitz R. The effect of hospital volume on the in-hospital complication rate in knee replacement patients. Health-Serv-Res. 1998; 33: 1191-210

50. Geraci J, Ashton C, Kuykendall D, Johnson M, Souchek J, del Junco D, Wray N. The association of quality of care and occurrence of in-hospital, treatment-related complications. Med Care. 1999; 37: 140-8

51. Geraci J. In-hospital complication occurrence as a screen for quality-of-care problems: what's next? Med-Care 2000; 38: 777-80

52. Thromboembolic Risk Factors (THRIFT) Consensus Group. Risks of and prophylaxis for venous thrombo-embolism in hospital patients. BMJ 1992; 305:567-74.

53. Broderick A, Mori M, Mettleman M, Streed S, Wenzel R. Nosocomial infections: validation of surveillance and computer modelling to identify patients at risk. Am J Epiderniol 1990; 131:734-42. 

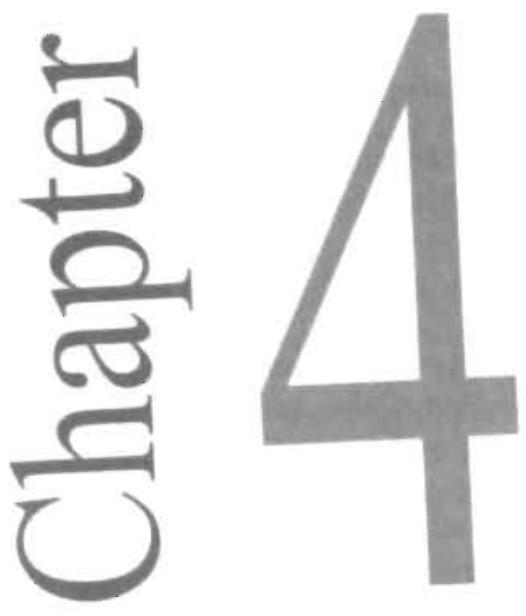

\section{The need to proceed}

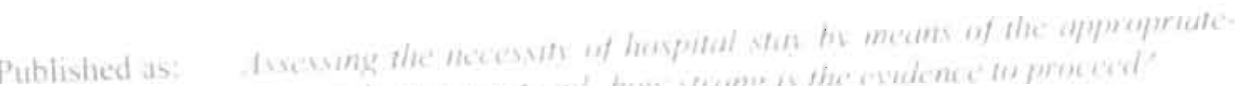

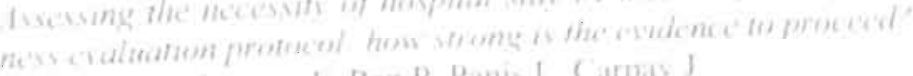
smeets P. Verheggen I. Pop P. Panis L. Carpay J Int d Qual Health Care, 2000: 12: 48.3-93. 


\begin{abstract}
Objective

To review the Appropriateness Evaluation Protocol (AEP) with respect to assessing the necessity of hospital stay at the University Hospital of Maastricht, a 700-bed university and regional hospital in the south of the Netherlands
\end{abstract}

\title{
Design
}

Literature search in Medline ${ }^{\circ}$ focusing on the validity, reliability and possibilities for intervention plus a description and the first results of pilot studies with a Dutch version of the Adult-Medical AEP (days of stay).

\section{Main outcome measures}

Face, content, and convergent validity, and reliability in terms of overall or specific: agreement and by kappa, of the Adult-Medical AEP when used by physicians and nurses.

\section{Results}

In comparison with other instruments, a comparatively good performance by the AEP has been reported in literature. Literature review revealed limitations in the AEP as a general and truly valid and reliable instrument for assessing the necessity of hospital stay. In applying a Dutch version (azM-AEP), we encountered similar difficulties.

\section{Conclusion}

Based on literature review and own findings, we conclude that the validity and reliability of the different versions of the AEP are not yet up to standard. Regarding the results of the interventions thus far, we do not encourage further use until additional improvements to both the instrument and the review conditions have been made. Perhaps a more disease-specific modulation and assessments based on computerized medical records could improve its applicability in clinical practice in general.

Key words; Appropriateness Evaluation Protocol, appropriateness, assessment, hospital care, necessity of stay, review. 


\section{Introduction}

Appropriateness ratings are perceived capable of revolutionizing health care. 'Potential purposes for utilization review (UR) are regulatory, research tool, education and quality improvement. ${ }^{2}$ The first study on necessity of hospital care is attributed to Codman (1916). ${ }^{3}$ In 1963 Querido found a rate of unnecessary use of hospital stay of $17 \%$ in the Netherlands. ${ }^{4}$ These kind of studies can be rated as UR as well as Quality Assurance (QA). ${ }^{3}$ Both methodologies assess medical care through application of defined criteria and/or expert opinion. QA focuses on effectiveness and covers structure, process and outcome, whereas UR merely concentrates on efficiency within the care process. In the United States, UR was first required with the institution of Medicare and Medicaid in 1965. Around $197690 \%$ of the hospitals had such a program. Appropriateness research has been stimulated in Europe by the "Concerted Action" of the European Union BIOMED program. ' Until 1975 UR focused on the length of stay. Since then, monitoring in UR has shifted towards the care setting. ${ }^{6}$ QA and UR both can be used in Utilization Management (UM), meaning: "the deliberate action by payers to influence hospitals or physicians or by hospital administrators to influence physicians, to increase the efficiency or the effectiveness with which hospital services are provided". 'Reasons for QA. UR and UM are: increasing costs of health care in general and hospital care in specific, assurance of the necessity and quality of care, efficient process management. an increasing influence of insurers and patients, and profiling of the hospital. Due to the social implications, it is important to stress the difference between necessity of care ("should the service be included in the care system?") and necessity of location ("is the setting appropriate?"). ${ }^{7}$ The latter is the subject of study in this article. Combining the care needed with the care provided three kinds of use can be distinguished: under use, balanced use and overuse.

QA/UR methods differ in their focus (diagnosis specific versus independent) and their use of criteria (implicit in the mind of the reviewer versus explicitly stated). 'implicit methods take into account the complexity of reality, but yield little insight into the internal considerations. They are also difficult to reproduce, since they are bound to the reviewers, who should be physicians. Implicit judgements by physicians are less reliable than those based on review instruments. ${ }^{8-9}$ Diagnosis specific methods are difficult to validate, since all the possible outcomes should be included. For efficiency reasons they are only applicable in frequently occurring disorders and treatments. ${ }^{10}$ Farlier research projects devised diagnosis independent instruments with explicit evaluation-criteria, e.g. the Standardized Medreview Instrument (SMI), the Intensity-Severity-DischargeCriteria (ISD) and the Appropriateness Evaluation Protocol (AEP). ${ }^{9}$ These instruments seem more reliable, more independent of the health care setting, and can be used by both physicians and nurses. The AEP has been most extensively used.

\section{Methods}

The aim of the medical audit committee of the university hospital Maastricht (azM) was to evaluate the applicability of the AEP for Quality Assurance (QA) and Utilization Review (UR) of hospital stay in the Dutch health care system. Five potential research areas can be distinguished in studying the appropriateness of medical care: dimensions, reliability, validity, potentials for use in practice and effects in terms of quality improvement and cost containment. "The value of QA/UR instruments is generally judged by their validity and reliability. " ${ }^{3}$ Validity comprises face-validity (recognizable), content-validity (comprehensiveness) and convergent-validity (concordance with expert panel). Reliability consists of intra-observer reliability (test-retest: repeated 
judgement by same observer), and inter-observer reliability such as overall agreement (all judgements; in fact a measure of association biased by unequal distribution and chance), specific agreement (judgements restricted to unnecessary care; still a measure of association biased by chance), and agreement corrected for chance (kappa). Our study is limited to the assessment of hospital stay with the Adult-Medical AEP. ${ }^{12}$ The Adult-Medical AEP also contains admission criteria. There also exist a specific Paediatrics AEP (PAEP) and Elective-Surgery AEP (SAEP), which we excluded from our study. Table 1 lists the differences between the original Adult-Medical AEP (day of stay section) and the Dutch azM-AEP. In the following text the hospital stay part of the Adult-Medical AEP will be referred to as 'AEP' and the Dutch azM version as 'azM-AEP'. A literature search was done via Medline ${ }^{\circ}$ (1966-1999; specific search terms in results section). Emphasis has been given to the validity, reliability and application characteristics. After the AEP had been translated and adapted to the Dutch situation, validity and reliability were also analyzed in a retrospective study covering different specializations (1994). ${ }^{13}$ After revision, reliability was studied concurrently in three specializations (1995). ${ }^{\text {If }}$ The unpublished results of this Dutch azM-AEP are also included.

\section{Resuits}

The study will be discussed in the following sequence: search results, validity and reliability and necessity of hospital stay according to the AEP and its different versions.

\section{Search}

Via Medine 70 potentially useable publications. were found using the search terms 'Appropriateness Evaluation Protocol' and 'Health-Services-Misuse/a!l subheadings' (54) combined with 'appropriateness' (16). Several of the studies included were national reviews. Thus the total number of studies in our search was in fact larger. Of the 70 studies 38 were excluded: 10 concerned the PAEP, two focused on the SAEP, 12 studied admissions only, and 14 (out of the 16 retrieved under 'appropriateness') dealt with methodological issues in measuring appropriateness, even though four of these mentioned the AEP. Of the 32 remaining studies addressing the appropriateness of stay measured by the AEP, five were in Spanish. This limited the accessibility of results to us. Two of these concerned surgical departments but did not mention whether they used the SAEP or another version. ${ }^{15-16}$ From a check of the references, four additional usable studies were found. ${ }^{17.20}$ Of the final total of 36 studies, three were restricted to methodological issues. ${ }^{4,20-21}$ 


\section{Validity}

Study of the validity of the AEP has been limited, whether it concerns face, content or convergent validity or use of the "override" option (ignoring the outcome of the instrument) (table 2). ${ }^{8-9,13 \cdot 14,18,22-24}$ The Spanish version too has been reported to yield valid results. ${ }^{26-26}$ The AEP has been found to have a high degree of sensitivity, but a low specificity. ${ }^{26}$ A better performance has been reported for the AEP and IntensitySeverity-Discharge-Criteria (ISD) in comparison with the Standardised Medreview Instrument (SMI) (table 3). "However, this conclusion was primarily based on assessing admissions and in the assessing the stay the override option was used in $25 \%$, whereas usually results are considered valid when the override is $5 \%$ or lower.

The AEP' induced over-reporting and the ISD and SMI under-reporting in comparison with a panel. The number of surgical days reviewed, was too small to draw final conclusions. Interpretation of validity of the AEP in comparison with these other instruments therefore requires caution. The azM-AEP yields an override of about $5 \%$, while physicians and nurses use the same criteria in order to justify hospital stay in $30 \%$ to $44 \%$. 14 This seems an acceptable outcome when using the instrument in terms of validity. Initially no differences were found in literature between concurrent and retrospective use of the AEP. ${ }^{8}$ Since then it has become clear, however, that the validity can be affected: in retrospective use by the quality of the record (especially items that have not been recorded), and in concurrent use by differences in information and time constraints (table 4). ${ }^{12-14,22,27-31}$ Use of additional information in 12\% to $75 \%$ of the cases reviewed has been reported in concurrent review. ${ }^{24},{ }^{12}$ Completeness of the medical record is associated with the necessity of stay , but has no independent influence. ${ }^{29}$ High agreement on the identical AEP-criterion used by different reviewers has been reported in literature with a $72 \%$ agreement $(95 \%$ CI $67-77 \%)$ and a kappa of $0.70 .^{10}$

\section{Reliabilit}

Only one study on the intra-observer variation was found ( 2 reviewers: overall agreement $85-88 \%$, kappa $0.50-0.58, \mathrm{p}<0.000 \mathrm{I})$. "Usually the inter-observer variation is measured by the overall agreement (mean AEP 75 - 98\%; azM-AEP 68 - 84\%), specific agreement (mean AEP 30 - 100\%; azM-AEP 25 - 36\%), or kappa (mean AEP 0.3I 0.94; azM-AEP 0.22 - 0.30)(table 5). ${ }^{8.9}, 13-14,17,20,23-24,30,13 \cdot 40$ The implicit judgement of physicians is less reliable compared to an assessment based on instruments like the AEP. ${ }^{8.9}$ In the azM-AEP the reliability among nurses was higher than among physicians. ${ }^{14}$ If -as in the azM studies- the medical and nursing records are not reviewed strictly on the basis of the AEP or the criteria for day care are not unequivocal, implicit judgement and associated lower reliability may occur. ${ }^{13-14}$ The reliability also varies if reviewers are not trained together. ${ }^{41}$ A thorough training course can yield a higher reliability (kappa $>0.70$ ). ${ }^{19.20,24}$ If the AEP is to be used for purposes other than screening or internal management, regular control measures of the reliability are recommended. " In a direct comparison with the AEP, the Medical Patient Assessment Protocol yielded higher reliabilities with an overall agreement of $98 \%(95 \% \mathrm{CI}: 96-99 \%)$, a specific agreement of $93 \%(95 \% \mathrm{CI}: 86-96 \%)$ and a kappa of $0.94 .{ }^{30}$ 
Table 1: Differences between the original Adult-Medical AEP (day of stay section) and the asM-AEP. 12
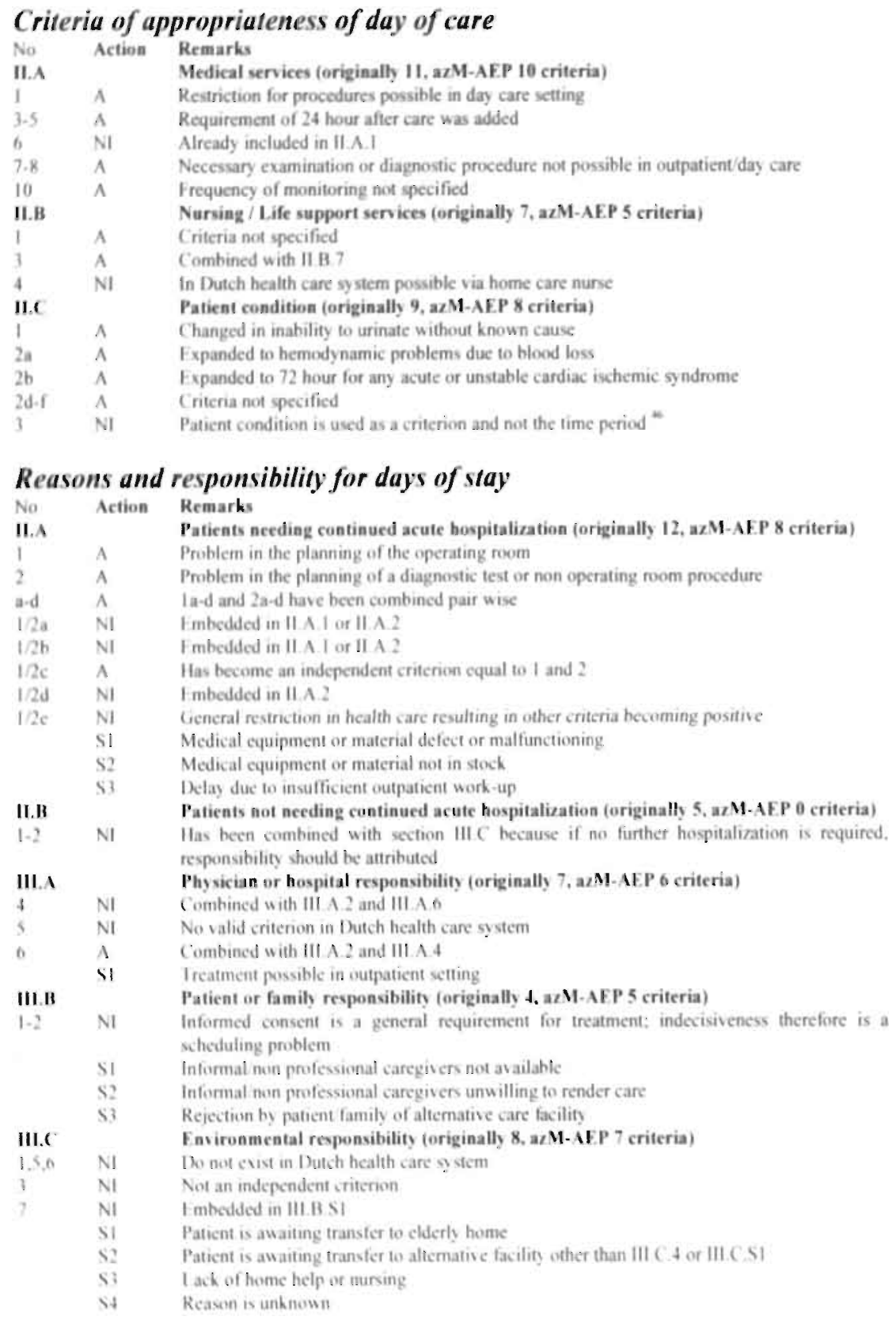

No. number of item in the original Adult-Medical AFP. A altered in the azM-AEP. NI not included in the azM-A P. S: supplemented in the azM-AFP 
Table 2: Validiny of the AEP

\begin{tabular}{|c|c|c|c|c|}
\hline Study and Year & Cases & Face & Content & Convergent \\
\hline \multicolumn{5}{|l|}{ US.A } \\
\hline $\begin{array}{l}\text { Gertmann \& Restuccia } \\
(1980)^{\prime}\end{array}$ & $100+100$ & Panel & Panel & $\begin{array}{l}2 \text { physicians + } 1 \text { nurse; } 2 \text { reviewer disagree- } \\
\text { ment, } 6-8 \% \text {, override } 5 \%(2-9 \%) \text {, happa over- } \\
\text { ride } 0.34-0.70 ; 3 \text { reviewer disagreement } 12 \% \text {. }\end{array}$ \\
\hline $\begin{array}{l}\text { Restuccia \& Payne } \\
(1981-1982)^{2}\end{array}$ & 72 & & & $\begin{array}{l}\text { AEP and panel of physicians specific agree- } \\
\text { ment } 55 \%\end{array}$ \\
\hline Strumwasser (1983) ' & 119 & & & Override AEP $25 \%$, ISD $5 \%$, SMI 1\% \\
\hline \multicolumn{5}{|l|}{ Italy } \\
\hline Apolone (1988) & & & & Override $7 \%$ \\
\hline \multicolumn{5}{|l|}{ Portugal } \\
\hline Bentes $(1984)^{4}$ & 100 & Panel & Panel & \\
\hline \multicolumn{5}{|l|}{ Spain } \\
\hline \multirow[t]{2}{*}{ Álonso (1996) } & 1988,778 & & & 1988, override 8\% \\
\hline & 1990.652 & & & 1990. override $6 \%$ \\
\hline Peiro $(1996)^{5}$ & 614 & & & $\begin{array}{l}\text { AEP and panel } 7 \text { reviewers: specific agree- } \\
\text { ment. }>61 \% \text {, kappa }>0.64\end{array}$ \\
\hline \multicolumn{5}{|l|}{ Turkey } \\
\hline Kaya $(1996)^{\circ}$ & 196 & Panel & Panel & Override $3.5 \%$ \\
\hline \multicolumn{5}{|l|}{ the Vetherlands } \\
\hline Schut (1994)? & 100 & Panel & Panel & Override $30 \%$ mostly physicians \\
\hline Esveld $(1995)^{*}$ & 74 and 45 & Panel & Panel & Override $5 \%$ and $4 \%$ \\
\hline \multicolumn{5}{|c|}{ 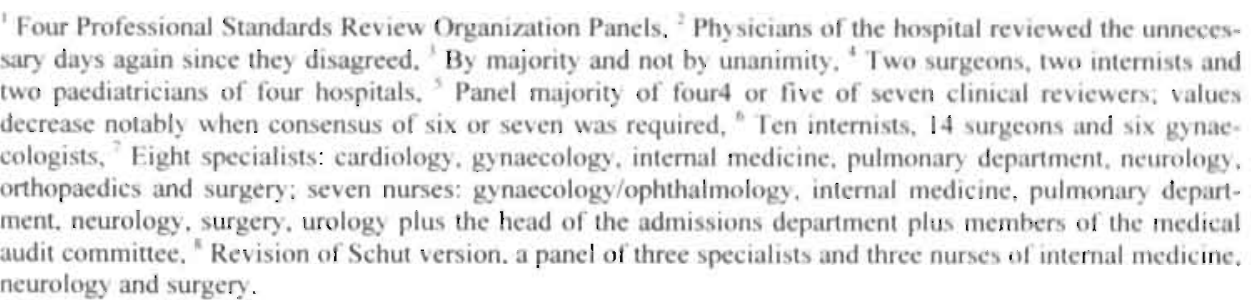 } \\
\hline
\end{tabular}

Table 3: Comparison of validity of the AEP. ISD and SMI

\begin{tabular}{|c|c|c|c|c|}
\hline $\begin{array}{l}\text { Kind of } \\
\text { panel. }\end{array}$ & $\begin{array}{l}\text { Unnecessary } \\
\text { instrument: panel ' }\end{array}$ & $\begin{array}{c}\text { Specific agreement } \\
\%\end{array}$ & $\begin{array}{l}\text { Kappa } \\
\text { Mean }\end{array}$ & $\begin{array}{c}\text { Kappa } \\
95 \% \text { confidence interval }\end{array}$ \\
\hline & FFS $\mathrm{HMO}^{2}$ & FFS / HMO & $F F S / H M O$ & $F F S / H M O$ \\
\hline AEP & $1.3 / 1.0$ & $59 / 72$ & $0.31^{\circ} / 0.47^{\circ}$ & $0.15-0.47 / 0.29-0.64$ \\
\hline ISD & $1.0 / 0.8$ & $60 / 64$ & $0.44^{*} / 0.43^{* *}$ & $0.26-0.62 / 0.26-0.61$ \\
\hline SMI & $0.6 / 0.5$ & $45 / 40$ & $0.31^{\circ} / 0.17^{\circ}$ & $0.14-0.49 / 0.01-0.33$ \\
\hline
\end{tabular}

ISD: Intensity-Severity-Discharge-Criteria, SMI: Standardized Medreview Instrument, FFS: Fee For Service, HMO : Health Maintenance Organization, 'Ratio between instrument and panel, ${ }^{2}$ FFS: seven internists and four surgeons with 9 to 15 years of experience of the fee-for-service hospital where the study was done; HMO: seven intemists and four surgeons with 6 to 25 years experience with the Kaiser group.

"p<0.001." p: 0.04. 


\section{Retrospective}

- Record has to be withdrawn from archives

- Record is complete, but should be blinded for the days following the reviewed day

- No additional information can be obtained from physicians and nurses

- Cannot be done by telephone

- Does not disturb the daily routine on the ward

- Less problems with time constraints

- Review by different reviewers does not need to be done on the same day

- Chance of bias due to information that has not been recorded

- Feedback has to be given by a report
Concurrent

- Record is usually directly available on the ward

- Data can be scattered over the medical record, the nurse record and by the bed of the patient and may still not be entered

- Additional information can be obtained from physicians and nurses

- Can be done by telephone

- Disturbs the daily routine on the ward

- Possible problems with time constraints

- Review by different reviewers needs to be done on the same day of assessment

- Chance of bias if the patients. own physicians and nurses conduct review (they are most informed about information that has not been recorded yet)

- Feedback. can be given directly

\section{Unnecessary hospital stay and affecting factors}

Unnecessary stays (table 6) may be due to different reasons of which only some can be influenced. ${ }^{8,11-14,18-19,22-24,31-12,36-39,42-43}$ Internal medicine and surgery usually have higher numbers of unnecessary hospital days. ${ }^{14,19,22,36,40,44-45}$ The outcome of the AEP can vary with the sampling technique, the length of stay and the part of the hospital stay that has been reviewed. ${ }^{\text {th }}$ Longer stays can be associated with higher numbers of unnecessary days, but this finding is not consistent. ${ }^{18,22,29,35,40,45}$ A general decrease in hospital stays does not reduce the percentage of unnecessary stays. ${ }^{40}$ However, the latter paris of hospital stays are certainly associated with higher numbers of unnecessary days (up $1066 \%$ in the day preceding discharge), ${ }^{18-19,29,32,39,43-47}$ Awaiting and discharge procedures as well as consultations seem to be substantial causes of unnecessary hospital stay. ${ }^{32,43,45}$ Feedback to physicians can lead to a small decrease in unnecessary days. 18.19, 25, 35-36, 40, 44, 46, 48 Feedback to the management has a modest effect only. ${ }^{40}$ The effect of feedback depends on the number of unnecessary days at the start of the feedback period, the level of feedback given and hospital characteristics. The effects of different kinds of feedback have also been compared: none, direct to physician, indirect via the supervisor of the physician, and judgement free to reviewer per case. ${ }^{40}$ With a maximum of four unnecessary days before the feedback intervention, the average length of stay could be reduced by half a day. Overall, judgemental and direct feedback proved to be the most effective, while both indirect and no feedback had any effect whatsoever. The outcome depends on the period of time the feedback procedure is in place, the system workload and environmental factors. Cost sharing for patients can decrease both the unnecessary and the necessary hospital days. ${ }^{17}$ Besides this, no relationship between unnecessary days and other variables have been found yet. A discharge coordinator improved the quality of the discharge procedure, but did not affect either the length of stay or the necessity of care as measured by the AEP. 


\begin{tabular}{|c|c|c|c|c|}
\hline Study and year & Cases (days) & $\begin{array}{l}\% \text { Overall } \\
\text { agreement }\end{array}$ & $\begin{array}{l}\% \text { Specific } \\
\text { agrecment }\end{array}$ & Kappa \\
\hline
\end{tabular}

\section{USA}

Siu (1974 - 1982)

Gertmann \& Restuccia $(1980)^{2}$

Wakefield $(=1980-1986)$

Strumwasser (1983)

Ludke + Booth (1986)

\section{Europe}

Bentes (1984)

Italy

Fellin (1990-1992)

Italy

Eggiman (1990 - 1991)

Switzerland

Eggiman (1993 - 1995)

Switzerland "

Chopard (1994 - 1995)

Bare (1995)

Spain

Alonso (1996)

Spain

Peiro (1996)

Spain

Kaya (1996)

Turkey "

Schut (1994)

the Netherlands

Esveld (1995)

the Netherlands

\section{Elsewhere}

Risphon (1983)

Israel

Paldi $(1992)=$

Mozes (1996)

Israc|

Zwarenstein ( 1989)

South Africa
6063

(56254)

\pm 113
$100+100$
850
75

20

n.s.

1847

880

$500(5665)$

44

\author{
1988:778 \\ 1990: 652 \\ $6 ! 4$
}

196

100

74

45

263

297

67
81 or 88

$92-94$

$88(3 r)^{\prime}$

90

AEP:

ISD:

SMI:

FFS panel

HMO panel:

75

$40-100$

$0.87-0.93$

$78-98$

$30-89$

Co: 87

Re: 86

84

1988:93

1990:93

.

93.96

68

70

$72 \rightarrow 71 \rightarrow 84^{11}$

86

$93(90-96)$

$71(66-76)^{6}$

0.78

0.63

Day care surgery is necessary/ unnecessary. ${ }^{2}$ Retrospective sub sample from 8000 patients from 41 hospitals. Three reviewers: two physicians and one nurse. 'Own calculation. 'Retrospective suh sample from 1266 admissions from all admissions from 21 hospitals. "Mean and $95 \%$ eonfidence interval scores. Variation between hospital and public health department. "Two reviewers who reviewed the same records concurrently $(\mathrm{Co})$ and one year later retrospectively (Re). "Overall results, individual results for internal medicine, surgery and gynaecology varied more ${ }^{10}$ Two specialists and two nurses of surgery, internal medicine and neurology departments: agreement within discipline $\rightarrow$ between different disciplines $\rightarrow$ from the group. * $p$ $0.001: \cdots p=0.001 ; \cdots p=<0.004 ;$ n.s: not specified 
Table 6: Results with the AEP

\begin{tabular}{|c|c|c|c|c|c|}
\hline Study and Year & $\begin{array}{l}\text { Cases } \\
\text { (days) }\end{array}$ & $\begin{array}{l}\text { IHS } \\
\text { (days) }\end{array}$ & MD & RN & Method \\
\hline \multicolumn{6}{|l|}{ USA } \\
\hline Siu $(1974-1982)$ & 1132 & $35 \pm 6 \%$ & 2 & & $\begin{array}{l}\text { Re, RS fay, } 6 \text { H representa- } \\
\text { tive for USA }\end{array}$ \\
\hline $\begin{array}{l}\text { Gertman \& Restuccia } \\
(1980)^{2}\end{array}$ & $100+100$ & $30 \%$ & 1 & 2 . & $\begin{array}{l}\text { Rel } 50+\text { Co50, sample day, } \\
\text { IUnTH }\end{array}$ \\
\hline $\begin{array}{l}\text { Restuccia \& Payne } \\
(1981 \text { - 1982) }\end{array}$ & 297 & $27 \%$ & & $\mathrm{x}$ & Re, RS day, IUrTH \\
\hline $\begin{array}{l}\text { Restuccia \& Gertmann } \\
\text { (1984) }\end{array}$ & 4928 & $20 \%$ & & $x$ & $\begin{array}{l}\text { Re, RS stratified sample of } \\
\text { admissions }, 24 \mathrm{H}\end{array}$ \\
\hline $\begin{array}{l}\text { Wakefield } \\
(=1980-1986)\end{array}$ & $\begin{array}{c}100 \\
(3342)\end{array}$ & $\begin{array}{l}38 \% \\
(21 \%)\end{array}$ & $x^{s}$ & $>1$ & $\begin{array}{l}\text { Re, all days, IVAMC } \\
\text { IUnTH, patients with hospi- } \\
\text { tal infection }\end{array}$ \\
\hline $\begin{array}{l}\text { Payne } \\
(1984-1985)\end{array}$ & 8049 & $41 \%$ & & $>1$ & Re, DBD. 6generalH \\
\hline $\begin{array}{l}\text { Booth } \\
(1986)\end{array}$ & $\begin{array}{c}6063 \\
(56254)\end{array}$ & $(48 \pm 2 \%)$ & & 26 & $\begin{array}{l}\text { Re, all days, sample from } 50 \\
\text { out of } 136 \text { VAMCs }\end{array}$ \\
\hline \multicolumn{6}{|l|}{ Europe } \\
\hline $\begin{array}{l}\text { Apolone (1988) } \\
\text { Italy " }\end{array}$ & 273 & $41 \%$ & 7 & & $\mathrm{Re}_{\text {. }} \mathrm{I}$ index day, InUnTH \\
\hline $\begin{array}{l}\text { Fellin }(1990-1992) \\
\text { Italy }\end{array}$ & 5435 & $28-49 \%$ & n.s. & n.s & $\begin{array}{l}\text { 4Re, } 2 \mathrm{Co}: 249 \mathrm{I}, 2 \text { unknown, } \\
\text { usually sample }\end{array}$ \\
\hline Alonso $(1988+1990)$ & $1988: 778$ & $1988: 21 \%$ & 1988:2 & & Re, all days of RS admission. \\
\hline Spain & $1990: 652$ & $1990: 22 \%$ & $1990: 2$ & & $1 \mathrm{TH}$ \\
\hline $\begin{array}{l}\text { Lorenzo (1991-1993) } \\
\text { Span }\end{array}$ & 1956 & $16-44 \%$ & & & $\mathrm{Re} 4 \mathrm{H}: 1345 . \mathrm{Co} 1 \mathrm{H}: 611$ \\
\hline $\begin{array}{l}\text { Martinez (1994) } \\
\text { Spain }\end{array}$ & $\begin{array}{c}249 \\
(1447)\end{array}$ & $32 \%$ & 4 & & $\begin{array}{l}\text { Re appendectomies. } \\
\text { 8general!! }\end{array}$ \\
\hline Bare (1995) & 639 & & 2 & & Re, all days of appropriate RS \\
\hline Spain & $(6371)$ & $(29 \%)$ & & & admissions, IUnTH \\
\hline $\begin{array}{l}\text { Ramos (1995) } \\
\text { Spain }\end{array}$ & 345 & $36 \%$ & $2 \mathrm{E}+2 \mathrm{I}$ & & $\mathrm{Re}_{\text {, }} \mathrm{RS}$ days, IUnTH: \\
\hline $\begin{array}{l}\text { Bentes }\{1990-1992\} \\
\text { Portugal }\end{array}$ & 7200 & $=46 \%$ & $=450$ & & $\begin{array}{l}\text { Re, RS day of RS admission. } \\
30 \mathrm{H}\end{array}$ \\
\hline $\begin{array}{l}\text { Eggiman }(1990-1991) \\
\text { Swiverland }\end{array}$ & $\begin{array}{c}4.309 \\
(41617)\end{array}$ & $\begin{array}{l}20 \% \\
(12 \%)\end{array}$ & $\geq 4$ & $\geq 1$ & Co, all days, 4 generalH. \\
\hline $\begin{array}{l}\text { Eggiman (1993-1995) } \\
\text { Switzeriand }\end{array}$ & 880 & $\begin{array}{l}\text { Co: } 10-21 \% \\
\text { Re: } 20-30 \%\end{array}$ & & & $\begin{array}{l}\text { Co, all days, same days } \mathrm{Re} \\
\text { one year later }\end{array}$ \\
\hline $\begin{array}{l}\text { Chopard (1994-1995) } \\
\text { Switzerland }\end{array}$ & $\begin{array}{c}500 \\
(5665)\end{array}$ & $28 \%$ & RA & & Co. all days. $1 \mathrm{Un} T \mathrm{H}$ \\
\hline $\begin{array}{l}\text { Houghtor ( } 1992-1993 \text { ) } \\
\text { United Kingóom. }\end{array}$ & 568 & $66 \%$ & & 1 & Co, day preceding discharge \\
\hline $\begin{array}{l}\text { Kaya }(1996) \\
\text { Turkey }\end{array}$ & 196 & 19 & 2 & 1 & $\begin{array}{l}\text { Co, } 1 \text { index day, } 1 \text { UnTH. } \\
\text { ITH. I generalH }\end{array}$ \\
\hline $\begin{array}{l}\text { Querido }(1957-1,959) \\
\text { the Netherlands }\end{array}$ & $\begin{array}{c}389 \\
(7773)\end{array}$ & $17 \%$ & I GP & & Co \\
\hline $\begin{array}{l}\text { Sehut ( [994) } \\
\text { the Netherlands. }\end{array}$ & 100 & $33 \%$ & $111 \mathrm{Pu}$ & 2 & $\begin{array}{l}\text { Re, DBD in weighted sample, } \\
\text { IUnTH }\end{array}$ \\
\hline $\begin{array}{l}\text { Fisveld ( } 1995) \\
\text { the Netherlands }\end{array}$ & 7445 & $\begin{array}{c}32 \% \\
24 \rightarrow 20 \rightarrow 33\end{array}$ & $\begin{array}{c}212 \mathrm{~N} \\
2 \mathrm{Su}\end{array}$ & 6 & Co. I index day, IUnTH \\
\hline
\end{tabular}




\begin{tabular}{|c|c|c|c|c|c|}
\hline Study and Year & $\begin{array}{l}\text { Cases } \\
\text { (days) }\end{array}$ & $\begin{array}{l}\text { IHS } \\
\text { (days) }\end{array}$ & MD & $\mathrm{RN}$ & Method \\
\hline \multicolumn{6}{|l|}{ Elsewhere } \\
\hline $\begin{array}{l}\text { Risphon (1983) } \\
\text { Israel }\end{array}$ & 236 & $17-31 \%$ & 2 & 1 & Re. IUnH I generalH \\
\hline $\begin{array}{l}\text { Paldi }(1992)= \\
\text { Mozes }(1996) \\
\text { Israel }\end{array}$ & 297 & $\approx 20 \%$ & $1 \mathrm{MS}$ & 1 & $\begin{array}{l}\text { Co, day before RS day, } \\
2 \text { Unlt }\end{array}$ \\
\hline $\begin{array}{l}\text { Zwarenstein (1989) } \\
\text { South Africa }\end{array}$ & 312 & $29 \%$ & $2 \mathrm{Su}$ & & $\begin{array}{l}\text { Co, sample } 23 \text { workdays. } \\
\text { IUnTH }\end{array}$ \\
\hline
\end{tabular}

In two of the six hospitals the nurse record also. "Maximum unnecessary days 4 , in only one of the 50 concurrent uses additional information of the hospital staff, ' 24 hospitals stratification: cast-west, urban-rural, small community-large community-teaching. 7 days of week, four seasons, 'Does not always lead to a longer stay. When in doubt the nurse handed, the record to the physician, "Minimum and maximum. 'Additional information none: $59 \%$, physician/nurse: $39 \%$, patient: $2 \%$, "Psychiatric patients included, "Always two physicians and one nurse. MD: physician. IHS: inappropriate hospital stay. Co: concurrent. DBD): day before discharge: always give higher perentages of unnecessary days than randomly selected days, E: Epidemiologist, G: Gynaecologist, GP: general practitioner, H: hospital, i: internist, MS: medical student, N: neurologist, n.s.: not specified, Pu: pulmonary specialist, RA: research assistants, Re: retrospective, RN: nurse, RS: randomly selected, Su: surgeon. T: teaching. Un: university, Ur: urban. VAMC: Veterans Affairs Medical Center.

\section{Discussion}

The discussion focuses on QA/UR as problem area, the validity and reliability of the AEP so far, the impact of review conditions, and possibilities to influence unnecessary hospital stay in the Dutch health care system. QA/UR is a difficult and sensitive problem area. This is illustrated by the ongoing debate on appropriateness of health care and the various terms used in AEP studies, such as: 'inappropriate', 'inactive' or 'unnecessary". ${ }^{50}$ Inappropriateness varies with time, place and the instrument applied. ${ }^{10}$ The measurability of the 'necessity of care' is questionable due to the relatively high likelihood of bias and overestimation, because necessary hospital days may be reviewed as unnecessary when the AEP is used. ${ }^{26,51}$ The necessity of hospital stay rate may not reach the ideal $100 \%$, because hospital beds can have different functions: for example, to provide a buffer to enable the optimal use of very expensive diagnostic procedures or treatments, to serve social care needs, and to form a back-up to other care sectors outside the hospital lacking either the medical knowledge or administrative procedures. ${ }^{\text {, } 10 .}$ 28. 38, 52 These inefficiencies might be considered transaction costs, needed to avoid much higher costs elsewhere in the hospital or health care setting. This is comparable to the problems and costs associated with operating a hospital at a $100 \%$ occupancy rate. ${ }^{47}$ Finally, some physicians, nurses and hospital management do not accept the AEP per se. ${ }^{2.13 .14,40}$ Especially if the AEP is being perceived as a check on the necessity of care or as a pure UR instrument, 'resistance' may occur. ${ }^{13-14,46}$ When the AEP is used as a QA instrument, professionals might also become disappointed if it is not specific enough to differentiate (e.g. not every patient can be operated in day care), yields little insight without further explanation (e.g. emphasizing "conservative medical management'), or diagnoses problems which cannot be influenced immediately (e.g. waiting list for nursing homes). In conclusion, an unequivocal answer to questions like "What does the AEP measure?" and "Can the necessity of hospital stay be assessed with a number of explicit -although general- criteria?" is still missing. 


\section{Validity}

Validating UM instruments like the AEP proves to be difficult. To a certain extent it surely is possible. The AEP seems to focus more on the hospital setting than on patient conditions. ${ }^{30}$ In relation to other versions, the azM-AEP' has a reasonable validity. A. relatively low use of the override option and frequent use of the same criteria for indicating necessity of stay by both physicians and nurses confirm this. Because of the impact of the sampling method, a random selection of one index day of the hospital stay from randomly selected admissions seems to be preferable. ${ }^{48}$ The influence of the part of the hospital stay also requires stratification. In their case mix, internal medicine and neurology might be expected to have a higher unnecessary stay than surgery. In validating instruments like the AEP (explicit criteria), a methodological problem occurs, since comparisons with panel or expert opinions introduce implicit criteria like professional standards and own opinions. ${ }^{4}$ Methods based. on reaching a consensus, among experts might indeed create new scientific insight, but also includes the danger of codifying old beliefs. "Because medical care is provided more often in day care, or on an outpatient basis or at home, the assessment of the necessity of hospital stay might be shifting to seriously ill patients and quantifiable clinical outcomes. ${ }^{10,30}$ The criteria may then become more objective (detailed), but at the same time less diagnosis-independent. This might introduce interference between the necessity of the care as such and the location where it is provided. ${ }^{7,10} \mathrm{It}$ also requires frequent adjustment with changes in clinical practice. 10 Maybe some other, more detailed 'delay tools' beyond that of physician discretion and more focused on individual patients are going to be used in future. ${ }^{53}$ In conclusion, relatively little is known about the validity of reviewing the necessity of stav by means of the AEP. especiallv in relation to the large-scale use of the AEP for this purpose to date. Further validation of the (azM-)AEP seems only possible after the reliability has been improved.

\section{Reliability}

The lower limits for overall and specific agreenent, and the kappa might be set at $80 \%$, $60 \%$ and 0.60 respectively. Further use of the (azM-)AEP would require a new study of its reliability, focusing attention on research conditions such as sampling. selection and instruction of the reviewers, concurrent versus retrospective application, criteria for day care, and ongoing reliability-analysis during training.

\section{Review conditions}

Although review conditions may influence the review process, this impact has not often been described. The minimum requirements for reporting as suggested by Ash are still relevant (table 7). ${ }^{48}$ Reviewing a medical and a nurse record takes about 10 to 20 minutes. ${ }^{8,3}$ Less use of time usually is associated with a lower reliability. ${ }^{8,13-14}$ Only one study has reported reliable results with assessment within 5 minutes. ${ }^{30}$ A thorough training of about four days seems necessary. 0, 20, 23, 33, 36-37, 46 Insufficient training diminishes the reliability. ${ }^{13.14,30}$ Reliability between the reviewers should already be measured during the training. ${ }^{20}$ Use of a recent day care list, in combination with an ASA classification, could prevent reliability problems from arising in this respect. During the assessment the reviewers should be released from their other duties. It Under these conditions, reliable application seems possible on a larger scale. ${ }^{20,21}$ 


\section{Necessary and unnecessary hospital stay and possibilities to influence}

The AEP has been used in many countries and settings, and at a professional, department and hospital level. Like other QA/UR instruments, the AEP also needs national adjustment and seems more appropriate for monitoring than for controlling purposes. ${ }^{2}$. 7. 10, 27 The AEP was not designed to assess individual patient situations, as was proved by study results. ${ }^{27}$ Specific problems with bias arise on several levels. ${ }^{54}$ On the hospital level these concern the comparability due to the kind of hospital, the number of beds and the case mix. On the departmental level these address differences in specialisation and the management. There is also a possibility of spill over bias, because colleagues of control and intervention departments can discuss the feedback. On the physician-level incomparability does not play a role anymore but the likelihood of spill over bias will increase considerably. The highest comparability and lowest bias are to be expected if physicians serve as their own controls before and after feedback. The size of the effect, however, can only be measured once. The time factor also introduces other kinds of bias such as changes in the patient population or in health care as residents rotate over departments and gain experience. Regarding the reasons for unnecessary stay that can be influenced by feedback (about $30 \%$ ) and earlier experiences, it is estimated that in the azM retrospective feedback to physicians can reduce unnecessary stay by at most $10 \%$ ( $3 \%$ of total stay). ${ }^{46}$ To prove this effect in a statistically sound way, approximately 6,000 days would need to be reviewed. " 5 Automated feedback via an electronic patient record seems cheaper than feedback by reviewers. ${ }^{10.56}$ However, in highly computerized settings these systems also still miss most unnecessary days (sensitivity 0.21 ), when compared to reviewers as a gold standard. ${ }^{21}$ Currently these methods seem only suitable for pre-selection purposes (positive predictive value 0.33 ). ${ }^{21}$ This may be caused by the reduction in accessible data when using an electronic record. Considerable inaccuracies ( $>20 \%$ in several categories) have been found in routinely collecting clinical data.

Table 7: Reporting requirements for studies of the necessity of hospital admussions and stay"

\section{Structured descriptions of the setting, with information about:}

A. Sources of data (credentials, general experience and explicit UR training of reviewers; data elements captured)

B. Study auspices (e.g. who is ordering/paying for these reviews? What is their understood purpose? Is participation voluntary?)

C. Sampling frame (e.g. the sampling unit, and exclusion of types of cases, wards or facilities)

D. Sampling design (a. clear description of what was done and the consequences of the strategy in terms of the over-representation of certain kinds of admissions/days)

E. Criteria for review (e.g. which instrument is used? Is timing as well as necessity of the procedure considered in determining the appropriateness of a surgical admission? Are review overrides allowed and, if so, what is being reported? )

\section{The distribution of sampled patients by:}

A. Hospital type (e.g. size, ownership, location (urban/rural), university affiliation)

B. Patient demographics

C. Service or ward (e.g. surgical versus medical)

D. Case-mix (e.g. obstetrics. versus psychiatric)

III. Rates of inappropriateness in each study by classification categories defined in II.

IV. A multivariate model to identify predictors of inappropriateness

(Possibly) the result of an agreed-upon multivariate model to identify predictors of inappropriateness (one for admission and a second one for days). This should heip distinguish common problem areas from idiosyncratic ones, and suggest areas in which collaborative work will he most fruitful 


\section{Conclusions}

Practising QA/UR is a matter of making choices. Several studies depict the AEP as a usable instrument to screen for unnecessary hospital stay and its reasons. It also enables users, to monitor the effect of feedback or other interventions. So far, however, the literature and our own Dutch pilot studies have revealed limitations in the AEP as a general and truly valid and reliable instrument for assessing the necessity of hospital stay. For the time being ${ }_{n}$ more direct methods focusing on changing test order behaviour and discharge planning seem to have a better acceptance and cost-benefit balance. ${ }^{58-59}$ But although these intervention programmes may be cheaper as such, their evaluation can be very costly as well. ${ }^{58}$ This, however, does not relieve us from the obligation to study QA/UR, the necessity of hospital care, and relevant measurement instruments. ${ }^{2}$ Perhaps care process analysis and continuous monitoring may provide opportunities for quality improvement. If a clinical process can be made transparent, and is not too complex, clinical performance indicators can be used to assess the quality of care and the necessity of the hospital setting. Also the choice for more diagnosis or specialisation dependent approaches -despite their inherent disadvantages- is still open. ${ }^{3,}$ Whether the AEP or AEP-like instruments are useful and desirable for quality assurance or utilisation review deserves further study and discussion. As such the efforts to develop a unified European AEP version will yield valuable information. ${ }^{60-61}$ 


\section{References}

1. Brook R. Appropriateness: the next frontier. Appropriateness ratings could revolutionise health care. BMJ 1994; 308:218-9.

2. Paccaud F, Guillain H. Should we assess appropriateness of care in Europe? Int J Qual Health Care 1994; 6:239-43.

3. Payne S. Identifying and managing inappropriate hospital utilization: a policy synthesis. Health Serv Res 1987; 22:709-69.

4. Querido A. Chapter 3: The hospital. In: The efficiency of medical care. A critical discussion of measuring procedures. Leiden: Stenfert Kroese NV; 1963. p. 122-143.

5. Liberati A, Apolone G, Lang T, Lorenzo S. A European project assessing the appropriateness of hospital utilization: background, objectives and preliminary results. The Project Steering Group and the Coordinating Center. Int I Qual Health Care 1995; 7:187-99.

6. Goldberg G, Hoolway D. Emphasizing "Level of Care" over "Length of Stay" in Hospital Utilization Review. Med Care 1975;XIII (6): 474-85.

7. Lavis $\mathbf{J}$, Anderson G. Appropriateness in health care delivery: definitions, measurement and policy implications. Can Med Assoc J 1996; 154:321-8.

8. Gertman P, Restuccia J. The appropriateness evaluation protocol: a technique for assessing unnecessary days of hospital care. Med Care 1981; 19:855-71.

9. Strumwasser I, Paranjpe N, Ronis D, Share D. Sel! L. Reliability and validify of utilization review criteria. Appropriateness Evaluation Protocol, Standardized Medreview Instrument, and Intensify-Severity-Discharge criteria. Med Care 1990; 28:95-111.

10. Restuccia J. The Evolution of Hospital utilization Review Methods in the United States. Int J Qual Health Care 1995; 7:253-260.

11. Black N. Appropriateness of medical care in Europe: a commentary. Int J Qual Health Care 1994; 6:231-2.

12. Restuccia J. Appropriateness Evaluation Reviewerst Manual. In: The AEP Final Report. Springfie!d, VA: National Technical Information Service, 1986 Publication No. PB87-112041.

13. Schut M. Appropriate use of hospita! beds. A study of the possibilities of the AEP. [in Dutch]. Maastricht University; 1994.

14. Esveld S. The Appropriateness Evaluation Protocol. A study of the reliability and validity of the AEP (in Dutch). Maastricht University; 1995.

15. Martinez Mas E, Peiro S, Meneu R, Rosello Perez M, Portella E. How many days of hospitalization for an appendectomy? Int J Qual Health Care 1994; 6:347-52.

16. Lapuente R, Bordonada R, et al. Adequacy of hospital admissions and stays of patients with uncomplicated inguinal hernia (in Spanish). Med Clin Barc 1997: I08:259-62.

17. Siu A, Sonnenberg F, Manning W. Goldberg G, Bloomfield E, Newhouse J, et al. Inappropriate use of hospitals in a randomized trial of health insurance plans. N Eng J Med 1983; 315:1259-66.

18. Restuccia J, Payne S, Lenhart G, Constatine H, Fulton J. Assessing the appropriateness of hospital utilization to improve efficiency and competitive position. Health Care Management Review 1987; 12:17-27.

19. Booth B, Ludke R, Wakefield D, Kern D, Burmeister L, Fisher E, et al. Nonacute Days of Care within Department of Veterans Affairs Medical Centers. Med Care 1991: 29:AS51-63. 
20. Ludke R, Wakefield D, Booth B, Kern D, Burmeister L. Nonacute Utilization of Department Veterans Affairs Inpatient Services. Background and Design of Nationwide Study. Med Care 1991; 29:AS29-39.

21. Nelson B, Gardner R, Hedrick G, Gould P. Computerized decision support for concurrent utilization review using the HELP system. J Am Med Inform Assoc 1994; 1:339-52.

22. Apolone G, Alfieri V, Braga A, Caimi V, Cestari C, Crespi V, et al. A survey of the necessity of the hospitalization day in an Italian teaching hospital. Qual Assur Health Care 1991; 3:1-9.

23. Bentes M, Gonsalves M, Santos M, Pina E. Design and development of a utilization review program in Portugal. Int J Qual Health Care 1995; 7:201-12.

24. Kaya S, Erdem Y, Dogrusoz S, Halici N. Reliability of a hospital utilization review method in Turkey. Int J Qual Health Care 1998; 10:53-8.

25. Baneres. J, Alonso J, Broquetas J, Anto J. Inadequate hospital admissions and inactive days of hospital stay in patients with chronic obstructive pulmonary disease and lung neoplasms [in Spanish]. Med Clin Barc 1993; 100:407-11.

26. Peiró S, Meneu R, Rosello M. Portella E, Carbonell Sanchis R, Fernandez C, et al. Validity of the protocol for evaluating the inappropriate use of hospitalization [in Spanish]. Med Clin Barc 1996; 107:124-9.

27. O'Neill D. Pearson M. Appropriateness of Hospital Use in the United Kingdom: a Review of Activity in the Field. Int J Qual Health Care 1995: 7:239-244.

28. Black N. Appropriateness of Hospital Use: The European BIOMED Project. European Collaboration on appropriateness of Hospital Bed Use: a Commentary (guest editorial). Int J Qual Health Care 1995; 7:185-186.

29. Ramos Cuadra A. Marion Buen J, Garcia Martin M, Fernandez Gracia J, Morata Cespedes M. Martin Moreno L, et al. The effect of completeness of medical records on the determination of appropriateness of hospital days. Int $\mathbf{J}$ Qual Health Care $1995 ; 7: 267-75$.

30. Mozes B, Rosenblum Y, Rom L, Friedman N, Shabtai E, Porat A. Medical patients assessment protocol: a tool for evaluating the appropriateness of utilizing hospitalstay days for acute medical patients; development, reliability and applications. Am J Med Qual 1996; 11:18-24.

31. Santos Eggimann B, Sidler M, Schopfer D, Blanc T. Comparing results of concurrent and retrospective designs in a hospital utilization review, Int J Qual Health Care 1997: 9:115-20.

32. Merom D, Shohat T, Harari G, Oren M, Green M. Factors associated with inappropriate hospitalization days in internal medicine wards in Israel: a cross-national survey: Int J Qual Health Care 1998; 10:155-62.

33. Wakefield D. Pfaller M. Hammons G, Massanari R. Use of the appropriateness evaluation protocol for estimating the incremental costs associated with nosocomial infections. Med Care 1987; 25:481-8.

34. Risphon S, Lubacsh S, Epstein L. Reliability of a Method of determining the Necessifv for Hospitalization Davs in Israel. Med Care 1986; 24:279-282.

35. Zwarenstein M. Benatar S. Shaw L. The pattern of care in the medical wards of a teaching hospital S Afr Med J 1990:77:460-3.

36. Santos Eggimann B. Paccaud F. Blane T. Medical appropriateness of hospital utilization: an overview of the Swiss experience. Int J Qual Health Care 1995; 7:227-32. 
37. Fellin G. Apolone G, Tampieri A, Bevilacqua L, Meregalli G, Minella C, et al. Appropriateness of hospital use: an overview of Italian studies. Int J Qual Health Care 1995; 7:219-25.

38. Bare M, Prat A, Lledo L, Asenjo M. Salleras L. Appropriateness of admissions and hospitalization days in an acute-care teaching hospital. Rev Epidemiol Sante Publique $1995 ; 43: 328-36$.

39. Paldi Y, Porath A, Friedman L, Mozes B. Factors associated with inappropriate hospitalization in medical wards: a cross-sectional study in two university hospitals. Int J Qual Health Care 1995; 7:261-5.

40. Alonso J, Munoz A, Anto J. Using length of stay and inactive days in the hospital to assess appropriateness of utilisation in Barcelona, Spain. J Epidemiol Community Health 1996; 50:196-201.

41. Santos Eggimann B. Hospital utilization reviews under field conditions: potential and improvements. Int J Tech Assess Health Care 1993;9:514-21.

42. Lorenzo S, Suñol R. An overview of Spanish studies on appropriateness of hospital use. Int J Qual Health Care 1995; 7:213-8.

43. Houghton A, Bowling A, Jones I, Clarke K. Appropriateness of admission and the last 24 hours of hospital care in medical wards in an east London teaching group hospital. Int J Qual Health Care 1996; 8:543-53.

44. Rishpon S, Epstein L, Rennert H. Unnecessary hospitalization days: rates in two general hospitals in Israel. Isr I Med Sci 1989; 25:392-7.

45. Chopard P, Perneger T, Gaspoz G, et al. Predictors of inappropriate hospital days in a department of internal medicine. Int J Epidemiology 1998; 27:513-9.

46. Payne S, Ash A, Restuccia J. The Role of Feedback in Reducing Medically Unnecessary Hospital Use. Med Care 1991; 29:AS91-106.

47. Restuccia J, Gertman P, Dayno S, Kreger B, Lehnart G. A comparative analysis of appropriateness of hospital use. Health Aff Millwood $1984 ; 3: 130-8$.

48. Ash A. The Design and Analysis of Hospital Utilization Studies. Int J Qual Health Care 1995; 7:245-252.

49. Restuccia J. The Effect of Concurrent Feedback in Reducing Inappropriate Hospital Utilization. Med Care 1982;XX:46-63.

50. Report of a working group prepared for the Director of Research and Development of the NHS Management Executive. What do we mean by appropriale heallh care? Quality in Health Care 1993; 2:117-123.

51. Phelps C. The methodological foundations of studies of the appropriateness of medical care. N Engl J Med 1993; 329:1241-5.

52. Grover P. Is Inappropriate Hospital Care an Inevitable Component of the Health Care System? Med Care 1991; 29:AS1-4.

53. Selker H. Beshansky J, Pauker S, Kassirer J. The Epidemiology of Delays in a Teaching Hospital. The Development and Use of a Tool That Detects Unnecessary Hospital Darys. Med Care 1989; 27:112-129.

54. Sacket D. Bias in Analytic Research. J Chron Diseases 1979; 32:51-63.

55. Schouten $\mathbf{H}$. The number of individuals needed to prove a difference between groups (in Dutch). Statistical bulletin for medicine and health care sciences. Maastricht University 1994; 1:4-7.

56. Nelson B. Gardner R. Decision support for concurrent utilization review using a HELP-embedded expert system. Proc Annu Symp Comput Appl Med Care $1993 ; 00195-4210$. 
57. Hobbs F, Parle J, Kenkre J. Accuracy of routinely collected clinical data on acute medical admissions to one hospital. Brit J General Practice 1997; 47:439-440.

58. Mannheim L, Feinglass J, Hughes R, Martin G, Conrad K. Training House Officers to be Cost Conscious. Effects of an Educational Intervention on Charges and Length of Stay. Med Care 1990; 28:29.39.

59. Winkens R. Improving test ordering in general practice. The effects of individual feedback [thesis]. Maastricht University;1994. p. 168.

60. Lorenzo S, Beech R, Lang T, Santos-Eggiman B. An experience of utilization review in Europe: sequel to a BIOMED project. Int J Qual Health Care 1999; 11:13-19.

61. Lang T, Liberati A, Tampieri A, et al. A European version of the Appropriateness Evaluation Protocol. Goals and Presentation. Int J Techn Assess Health Care 1999; $15: 185-97$. 

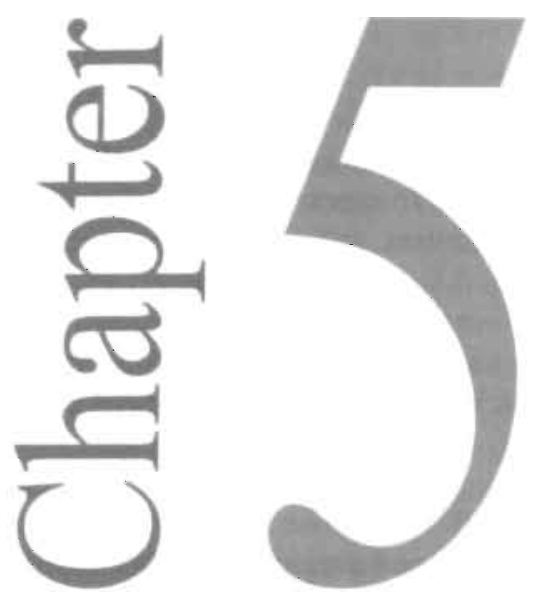

\section{To stay or not to stay}

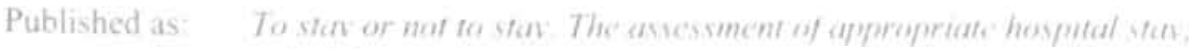
a Duth report

Panis L. Pop P. Verheggen F

Int J Qual Health Care, 2002: 14: 55-67. 


\begin{abstract}
Objective:

To adjust the adult-medical Appropriateness Evaluation Protocol (A.EP) into a valid and reliable instrument for use in the Dutch health care system, to assess the appropriateness of hospital stay and to identify the causes of inappropriateness.
\end{abstract}

\title{
Design:
}

The appropriateness of hospital stay was assessed in a cross-sectional survey on a sample of over 4500 days of stay using a modified, Dutch version of the Appropriateness Evaluation Protocol (DAEP).

\section{Setring:}

The appropriateness of stay was assessed in five internal and surgical departments for adult acute care in The university hospital of Maastricht, a 700-bed hospital with a teaching and regional function, located in the southern part of the Netherlands.

\section{Results:}

The results showed that over $20 \%$ of the hospital stay was inappropriate. Half of the inappropriate hospital stay $(45.1 \%)$ was due to (internal) hospital procedures. The DAEP proved to be valid $(\kappa=0.76 ; 95 \%$ confidence interval $(95 \% \mathrm{CI}): 0.68-0.84)$, reliable $(\mathrm{K}=0.84 ; 95 \% \mathrm{CI}: 0.75-0.93)$ and easy to use.

\section{Conclusion:}

A substantial proportion of hospital stay was found to be inappropriate, due to hospital procedures and the inability to refer patients to other care facilities or care providers. The D-AEP can be used for monitoring the appropriate hospital stay and in detecting possible causes of inappropriate stay. Analysis of the causes of inappropriate hospital stay provided useful data for improvement actions.

Key words: Appropriateness of hospital stay, appropriateness evaluation protocol, DAEP, reliability, validity. 


\section{Introduction}

\section{The Dutch health care setting.}

The Dutch constitution requires that the government guarantees the availability of high quality public health care. This implies continuous attention of the government for the quality, the accessibility and the (reasonable) pricing of this health care, to ensure access to good, affordable health care for every citizen. In the past, legislation regarding the financing of this health care could follow the pace of medical developments. However, during the seventies emphasis had to be laid on the control of health care costs. Three main issues were set in the policy of cost containment: planning of health care facilities, in order to control the capacity, price-fixing, in order to control the costs and the capacity of health care and the financing of that health care, in order to spread the costs of health care fairly. Today, there are 3.6 hospital beds per 1,000 inhabitants, nationwide. The Dutch government aims to reduce this number to 2.0 by the year 2005 . The overall mean length of stay for acute care is 9.2 days. 'Due to the cost containing actions, as mentioned above, waiting lists for non-acute in-patient care arose in the seventies and are still growing. Thus, the remaining hospital beds have to be used as efficiently as possible. One way to achieve this is to avoid inappropriate (hospital) stay or, at least, to keep it to a minimum. In order to assess the (in)appropriateness of hospital stay in the Dutch health care setting, a specific decision aid was needed. The Appropriateness Evaluation Protocol (AEP) ${ }^{2-3}$ has already proven its use in US and other European health care settings. ${ }^{4.18}$ The original AEP (US-AEP) is a criteria-based decision support tool for determining the medical necessity of hospital admissions and days of care. It consists of sets of generic (diagnosis-independent) and explicit (care related) criteria for adult medical-surgical, pediatric and elective surgery cases. The AEP consists of a criteria list for appropriate stay and a list of reasons for inappropriate stay. If the criteria cannot be met, the list of reasons indicates why the patient is nevertheless staying in the hospital. The instrument includes a so-called "override option', which means that even if a patient does not satisfy the defined criteria for appropriate stay, the assessor can, with solid arguments, override the AEP protocol and classify the hospital stay as appropriate.

\section{Previous experiences with the AEP.}

In 1994, the Peer-Review Committee of the university hospital of Maastricht initiated a study ${ }^{19-23}$ of more efficient use of hospital beds, based on the A.EP. The first aim of that study was to evaluate whether the 'medical-adult AEP for clinical stay' was useful in a Dutch clinical setting and to modify the instrument, if necessary. During this project, the US-AEP was modified into a preliminary Dutch model of the AEP (azM AEP). The results however yielded highly diverging conclusions. While it was felt that this instrument had great potential to assess (in)appropriate stay, the level of agreement between the users of the decision aid was poor $(\kappa=0.30)$. Some of the criteria were found to be obsolete. Among the problems encountered were: diverging conclusions due to differences in filing methods, ambiguities about outpatient treatment, differences of opinion among observers and unclear or incomplete registration of medical and nursing data. ${ }^{22-23}$ In addition, users felt that the instrument insufficiently addressed aspects relating to nursing care. For a diagrammatic representation of this previous experience please refer to Figure 1. Thus, it was concluded that the pre-DAEP had to be improved, taking into account the (methodological) problems encountered in the pilot study. 


\section{Study objectives}

In 1998, the Peer-review Committee decided to evaluate the AEP's usefulness in a follow-up project. The objectives of this study were:

- To modify the AEP based on the experiences of the previous project;

- To assess the validity and the reliability of this modified, Dutch AEP (DAEP);

- To measure the extent of (in)appropriate hospital stay;

- To identify the causes associated with inappropriate stay.

The instrument aimed to assess the appropriateness of the setting, not the appropriateness of care. ${ }^{24}$ Neither the appropriateness of patient admissions nor the appropriateness of professional services were to be assessed. The instrument had to be easy to use and had to be applicable to both utilization review ${ }^{25}$ and continuous quality improvement.

\section{Methods}

\section{Definition}

Appropriate hospital stay (the setting) was defined as: "In-patient stay, requiring continuous and active medical, nursing or paramedical treatment, which under existing legislation can not be provided through extramural care, daycare or outpatient care"

\section{Instrument modification \& application}

In this study, the standard medical-surgical AEP was used as source instrument. For the use in the Dutch health care setting, the criteria list and the reason list were altered. Regarding the previous experiences on the azM-AEP it was initially decided to develop specialty-specific AEP's (ss-AEP). The criteria for appropriate stay were based on reviews of clinical interventions, from which outpatient interventions were excluded. The modification of the criteria was carried out in close cooperation with physicians and nursing staff. Delphi techniques were used to modify the criteria and reasons where necessary. A literature study ${ }^{26-27}$ showed that retrospective application of the AEP using patient files tended to yield higher percentages of inappropriate stay. Therefore the ssAEP's were applied concurrently. This allowed the reviewers to ask additional questions. These ss-AEP's were tested at the obstetrics, gynecology and neurology departments. A total number of 733 days of stay (192 for obstetrics, 240 for gynecology and 301 for neurology) was assessed. An evaluation of the resulting data revealed a high level of correspondence between the various ss-AEP's, so one uniform, preliminary model of the DAEP (pre-DAEP) was developed. Later on, pre-DAEP was condensed into a final model: the DAEP. to improve the user-friendliness of the instrument (Appendix 1).

\section{Study sampling and data collection}

The study was carried out at the university hospital of Maastricht (the Netherlands), a 700 -bed teaching hospital. Yearly, this hospital generates about 200,000 days of stay with 22,000 admissions and a mean length of stay of 9,0 days. In the participating wards (surgery, internal medicine, obstetrics, gynecology, neurology and neurosurgery), the stay of each patient, present on the index-day, was assessed by a concurrent application of the DAEP list of criteria. These wards were selected on one hand as a fair reflection of the general patient population; on the other hand, the surgical, internal and neurological ward had already participated in the previous AEP project. ${ }^{22-23}$ Since the neurological ward in the previous AEP project showed a high rate of inappropriate stay. 
other wards (obstetrics, gynecology and neurosurgery), with an expected low rate of inappropriate stay, were added. By doing this, the instrument could be tested on wards with high and low rates of inappropriate stay. The index-days were selected at random over two six-months periods (Oct. 1998 - Apr. 1999 and Aug. 1999 - Feb. 2000). On average 3.4 days per week, evenly distributed over weekdays and weekends were evaluated. Only the index-day was assessed, not the entire hospitalization. Reviewed data included date of index-day, patient registration number, ward and score on criteria or reason list. The assessment of the index-day was done after the physicians had completed their ward round. During this round medical and nursing information is exchanged and discussed and the treatment plan is specified. This exchange of information and recording of the treatment policy promotes unambiguous assessments by both physicians and nursing staff of the appropriateness of the patients' stay. This was expected to raise the level of agreement between physicians and nursing staff in their assessment.

\section{Reliability \& Validity}

The review was carried out by two groups. The first group consisted of registered nurses $(\mathrm{n}=53$ ) of the participating wards. At the same time and independently from the first group, the second group, consisting of physicians $(n=9)$ of the participating wards, carried out a review of a randomly selected sample of the index days $(n=143)$. Both groups were trained in the use of the questionnaire by the research team. This training implied guidelines for use of the instrument. The inter-rater reliability was calculated from the level of agreement between the results obtained by the nursing staff and the physicians. To avoid possible Hawthorne effects and to increase the inter-rater reliability, only data already registered in the patient record(s) were to be used to score one or more criteria of the DAEP. Since the inter-rater agreement was high (see results section) and the possible sources of error contributing to inter-rater reliability, to our opinion, were taken into account, ${ }^{28}$ we did not assess the intra-rater reliability in this study, although previous experiences on the intra-fater reliability from the first project (fig. 1) were taken into account. ${ }^{23}$ During the design phase, the (content) validity of the AEP-criteria list was increased through the evaluation of these criteria by the physicians and nursing staff of the participating departments. The physicians and nursing staff evaluated the criteria independently from each other. Their comments were used to adjust or delete criteria or to add new criteria. Consensus about these criteria was established through three Delphi rounds. In order to assess the (content) validity, the data collected using this criteria list were compared with the opinions of other department's physicians and nursing staff not using the AEP. The frequency of the use of the 'override option' provides an indication about the (content) validity of the instrument. A low percentage of override cases was thought to contribute ${ }^{24}$ to the validity of the instrument.

\section{User-friendliness}

To assess practical value of the AEP. the time, needed to complete the review of one index-day, was measured. 


\section{Statistical analysis}

The data concerning criteria for appropriate stay and reasons for inappropriate stay were assessed using descriptive analysis. To assess the inter-rater agreement between physicians and nursing staff, the Kappa value was calculated. This $\kappa$ - statistic describes the true agreement as a proportion of the potential agreement, after correction for coincidental agreement. The Pearson Chi-square test was used to test the significance of differences in the rate of appropriateness between groups. All data analysis was performed using SPSS-PC software package, version 8.0.

Fig. 1: Synopsis of the AEP-project

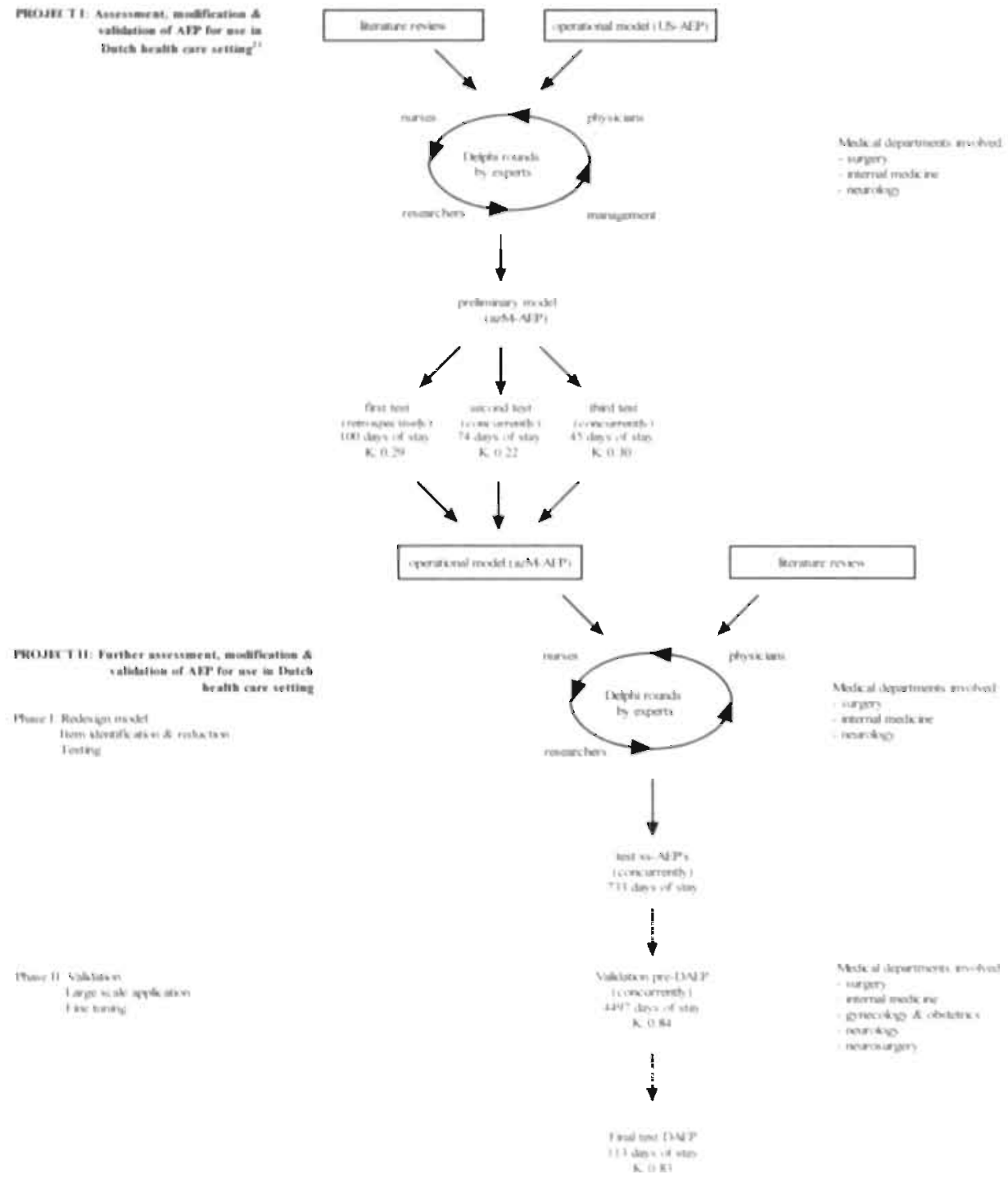




\section{Results}

A total of 4578 days of stay were assessed. Data on 81 days of stay $(1.8 \%)$ were found to be incomplete and had to be excluded from the analysis. Thus, a total of 4497 days of stay were analyzed, distributed over 5 medical departments, involving 1528 patients using 219 beds (table 1).

Table 1: Sample size by medical department

\begin{tabular}{lccc}
\hline \multicolumn{1}{c}{ Medical department. } & Hospital beds & Patients & Days of stay \\
\hline Surgery & 81 & 408 & 991 \\
Internal medicine & 56 & 176 & 899 \\
Obstetrics \& Gynecology & 38 & 480 & 1167 \\
Neurology & 26 & 31.4 & 94.4 \\
Neurosurgery & 18 & 150 & 496 \\
\hline Total & 219 & 1528 & 4.497
\end{tabular}

\section{Reliability}

The level of agreement between the results obtained by the nursing staff and the physicians regarding the appropriateness of stay was high, both with the pre-DAEP ( $\mathrm{K}=$ $0.84 ; 95 \%$ confidence interval $(\mathrm{CI}): 0.75-0.93)$ and with the DAEP $(\mathrm{k}: 0.83 ; 95 \% \mathrm{Cl}$ : $0.69-0.97)$.

\section{Validity}

The level of agreement between the groups regarding the selection of criteria to be included and the comparison between the (expert) opinions and the actual 'score' by the DAEP was found to be high $(k=0.79 ; 95 \% \mathrm{Cl}: 0.65-0.93)$. The 'override option', as mentioned above, was used in only $2.3 \%(n=104)$ of the total number of days of stay evaluated in the present study.

\section{User-friendliness}

After tailored on-site training, the assessors took an average of 23 seconds (range: 10 72) to assess one day of stay using the DAEP. The subjective opinion of the observers was that at first use of the instrument it required some training, but afterwards it allowed them to assess the appropriateness of stay easily and unambiguously.

\section{Proportion of inappropriate days of stay}

In the study sample over a quarter $(27.4 \%)$ of the days of stay was inappropriate. Table 2 shows the amount of inappropriate stay, providing both the percentage of inappropriate stay and an estimation of the total inappropriate stay on an annual basis (expressed as inappropriate days of stay). Since there were considerable differences between the specialties in the percentages of inappropriate stay, these percentages were corrected for outliers, resulting in an overall percentage of inappropriate stay of $20.6 \%$ $(95 \%$ Cl: $18.2-23.2)$. Based on this conservative estimate, the estimated inappropriate days of stay on annual base would amount to a total of 19,035 days. 
Table 2: Inappropriate days of stay by medical department

\begin{tabular}{lcccc}
\hline Medical department & Days of stay & $\begin{array}{c}\text { Inappropriate } \\
\text { days of stay } \\
(n)\end{array}$ & $\begin{array}{c}\text { Inappropriate } \\
\text { days of stay } \\
(\%)\end{array}$ & $\begin{array}{c}\text { Annual inappropriate } \\
\text { days of stay } \\
(n)^{\prime}\end{array}$ \\
\hline Surgery & 991 & 265 & 26.7 & 9599 \\
Internal medicine & 899 & 127 & 14.1 & 4575 \\
Obstetrics \& Gynecology & 1167 & 107 & 9.2 & 1129 \\
Neurology & 944 & 623 & 66 & 8530 \\
Neurosurgery & 496 & 105 & 21.2 & 1363 \\
\hline Total & 4497 & 1227 & 27.3 & 25196 \\
& & & 20.6 &
\end{tabular}

Pearson Chi-square: 993.9, df: 4. $p<0.001$. 'Estimation based on figures 1998. 'Corrected for outliers

\section{Factors associated with inappropriate stay}

The reasons for inappropriate stay are given in table 3. More than half $(51.5 \%)$ of the inappropriate stay is caused by external factors (i.e. factors that cause delay in patient's discharge or transfer and who cannot be controlled by the hospital). Within these external factors, the unavailability of other care facilities accounts for the major part $(73.4 \%)$ of this inappropriate stay. This problem especially occurs within the department of neurology where $52.2 \%$ of their inappropriate stay is caused by this unavaliability of other care facilities. Also in the departments of surgery and neurosurgery approximately one third $(35.8 \%$ and $32.3 \%)$ of the inappropriate stay is related to the unavailability of other care facilities. The other half of the inappropriate stay $(45.1 \%)$ is due to internal factors (i.e. delays due to internal (hospital) procedures that, in principle, can be controlled by the hospital itself.

With regard to the delay in discharge procedures, differences can be found among the medical departments involved. The high percentage of this inappropriate stay in the obstetrics \& gynecology department is mainly attributable $(94 \%)$ to the medically indicated admission of the newborn, while the mother has no medical reason for further stay. The use of care supervisors within the neurology department explains the low percentage of this inappropriate stay. Among other things, these care supervisors control the discharge procedures. Three factors (unavailability of other care facilities, delay in discharge procedures and delay in therapy / diagnostics) account for $78.9 \%$ of the overal! inappropriate stay. The internal factors for inappropriate stay are specified in table 4 and 5 . Table 4 shows that the main reason for inappropriate stay by internal factors is the delay in discharge procedures $(38.1 \%)$. There were insufficient data to point out the exact cause of this delay. The delay in diagnostic procedures accounts for $34.4 \%$ of the inappropriate stay by internal factors. Table 4 and 5 also provide an estimate of the annual inappropriate stay. If the inappropriate stay is seen in terms of need for further hospitalization, $73.8 \%$ of the days $(\mathrm{n}=905)$ rated as inappropriate were judged by the reviewers as not needing further in-patient care (fig 2). The setting ${ }^{24}$ of these days was inappropriate. These patients could have been treated outside an acute care hospital setting. 


\begin{tabular}{|c|c|c|c|c|c|c|c|c|}
\hline \multirow{3}{*}{$\begin{array}{l}\text { Medical } \\
\text { department }\end{array}$} & \multicolumn{4}{|c|}{ External factors } & \multicolumn{4}{|c|}{ Internal factors } \\
\hline & $\begin{array}{c}\text { lack of } \\
\text { wolunteer aid }\end{array}$ & $\begin{array}{l}\text { Primary care } \\
\text { not available }\end{array}$ & $\begin{array}{l}\text { Other can: } \\
\text { facilities not } \\
\text { available }\end{array}$ & $\begin{array}{l}\text { Delay in } \\
\text { discharge } \\
\text { procedures }\end{array}$ & $\begin{array}{l}\text { Delisy in } \\
\text { therapy } \\
\text { Diagnostics }\end{array}$ & $\begin{array}{l}\text { Test results } \\
\text { not available }\end{array}$ & Orher' & $\begin{array}{c}\text { Total } \\
(100 \%)\end{array}$ \\
\hline & ${ }^{n}$ & $\begin{array}{l}n \\
(\infty)\end{array}$ & $\begin{array}{c}n \\
(13)\end{array}$ & $\begin{array}{l}n \\
+\infty\end{array}$ & $\begin{array}{l}n \\
x=1\end{array}$ & $\begin{array}{c}n \\
\left(n_{0}\right)\end{array}$ & $(\%)$ & $n$ \\
\hline Surgery & $\begin{array}{l}24 \\
(9.1)\end{array}$ & $\begin{array}{l}28 \\
(10.6)\end{array}$ & $\begin{array}{c}95 \\
(35.8)\end{array}$ & $\begin{array}{c}64 \\
(24.1)\end{array}$ & $\begin{array}{c}36 \\
(13.6)\end{array}$ & $\stackrel{4}{(1.5)}$ & $\begin{array}{c}14 \\
(5.3)\end{array}$ & 265 \\
\hline Internal medicine & $\begin{array}{c}10 \\
(7.9)\end{array}$ & $\begin{array}{l}12 \\
(9.5)\end{array}$ & $\begin{array}{c}8 \\
(6.3)\end{array}$ & $\begin{array}{c}36 \\
(28.3)\end{array}$ & $\begin{array}{l}40 \\
(31.5)\end{array}$ & $\begin{array}{c}15 \\
(11.8)\end{array}$ & $\begin{array}{c}6 \\
(4.7)\end{array}$ & 127 \\
\hline $\begin{array}{l}\text { Ghstetrics d } \\
\text { Gynecology }\end{array}$ & $\begin{array}{c}13 \\
(12.1)\end{array}$ & $\begin{array}{c}1 \\
(1.0)\end{array}$ & $\begin{array}{c}1 \\
(1.0)\end{array}$ & $\begin{array}{c}67 \\
(62.6)^{4}\end{array}$ & $\begin{array}{c}12 \\
(11,2)\end{array}$ & $\begin{array}{c}10 \\
(9.3)\end{array}$ & $\begin{array}{c}3 \\
(28)\end{array}$ & 107. \\
\hline Neumlogy & $\begin{array}{l}32 \\
(5.1)\end{array}$ & $\begin{array}{c}33 \\
(5.3)\end{array}$ & $\begin{array}{l}326 \\
(522)\end{array}$ & $\begin{array}{c}36 \\
(5.8)\end{array}$ & $\begin{array}{c}165 \\
(26.5)\end{array}$ & $\begin{array}{r}18 \\
(2.9)\end{array}$ & $\begin{array}{c}13 \\
(2.1)\end{array}$ & 623 \\
\hline Neunsurgery & $\begin{array}{c}1 \\
(0.9) \\
\end{array}$ & $\begin{array}{c}14 \\
(13.7)\end{array}$ & $(32.3)$ & $\begin{array}{c}28 \\
(26.5)\end{array}$ & $\begin{array}{r}20 \\
{[19]}\end{array}$ & $\begin{array}{l}2 . \\
(1.9)\end{array}$ & $\begin{array}{c}6 \\
(5.7) \\
\end{array}$ & 105 \\
\hline Total & $\begin{array}{c}80 \\
(6.5)\end{array}$ & $\begin{array}{c}88 \\
(7,2)\end{array}$ & $\begin{array}{c}464 \\
(37.8)\end{array}$ & $\begin{array}{l}231 \\
(18.8)\end{array}$ & $\begin{array}{c}273 \\
(223)\end{array}$ & $\begin{array}{c}49 \\
(4.0)\end{array}$ & $\begin{array}{c}43 \\
(3.4)\end{array}$ & 1227 \\
\hline
\end{tabular}

Pearson Chi-square for exremal fạciors only: 146.102, df 8, p < 0.001 . 'Pearson Chi-square for internal factors only: 139.907 , df: 8 , p < 0.005 . Pearon (hi-square fir all factors: 388.8 . df: $24, \mathrm{P}<0.001$. "94\% of this delay is attritutable to a medical reason for admission of the neonate(s), while the mother has no medical reason for admission 
Fig. 2: Inappropriate stay, specified by the need for further hospital stay

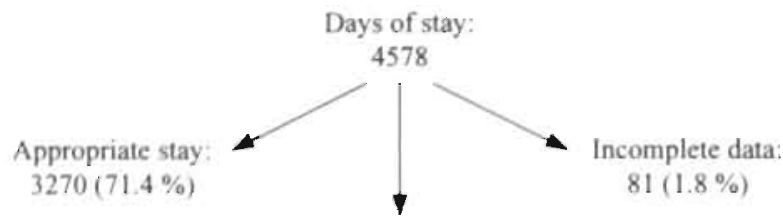

Inappropriate stay:

$1227(26.8 \%)$

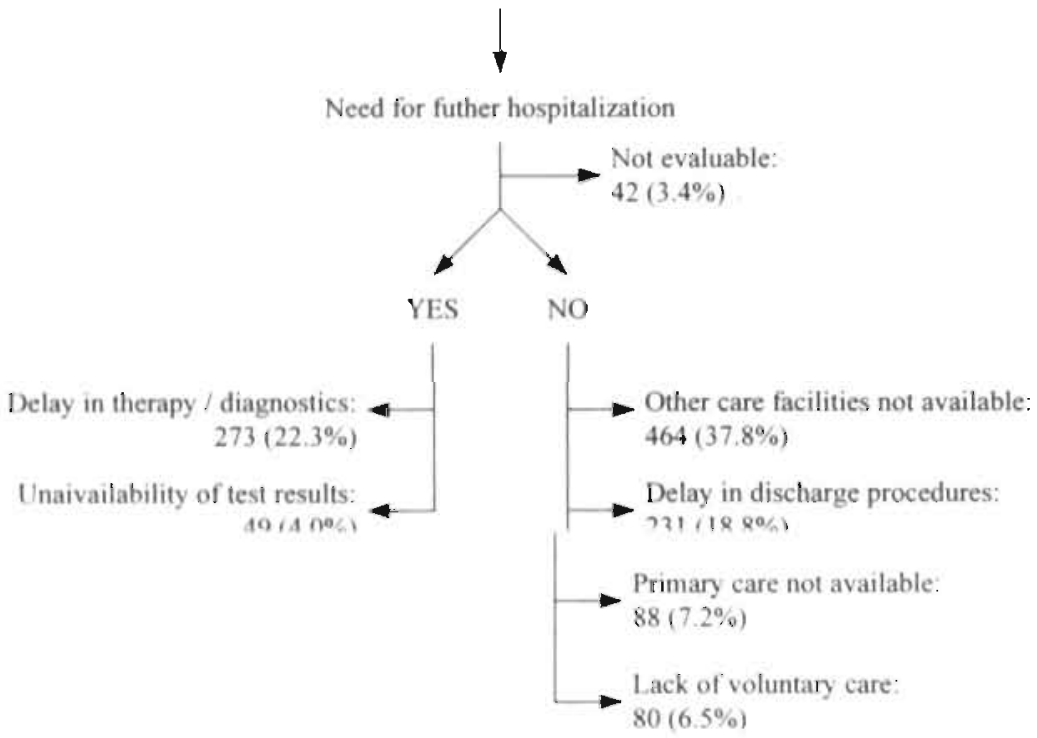

\section{Discussion}

The DAEP was developed in order to produce a valid and reliable, as well as easy to use tool for assessing the appropriateness of hospital stay. Since the criteria for appropriate stay in the DAEP were formulated rather strictly, we expected the percentage of inappropriate stay to be higher than that found in other studies. 4.18 However, the percentages of inappropriate stay we found in this study are in close agreement with those in studies performed in other countries. The US-AEP and the DAEP are compared in appendix 2.

As seen in the Method section, the causes of inappropriate stay can be divided into two headings: external and internal factors. External factors cause delays in a patient's discharge or transfer by factors over which the organization has little control. 'Blocked beds ${ }^{30.31}$ (patients staying in the hospital without medical indication and with, as yet, no options for transfer to other care facilities) are an example of these factors. Internal factors on the other hand, can be controlled by the organization. These factors usually concern inefficiencies in medical treatments and / or patient logistics. Internal factors. are mostly related to organizational problems (inefficient operational management). The groups of internal and external factors separately can be divided into three main. categories. For external factors there are: a lack of volunteer aid, a (temporary) lack of 
primary care facilities and a lack of options for transfer to other care facilities. For internal factors these include delay in discharge planning, delay in the applications for or planning of diagnostics and / or therapy and delay due to unavailability of test results.

Table 4: Specification inappropriate days of stay related to internal factors

\begin{tabular}{clcc}
\hline $\begin{array}{c}\text { Internal } \\
\text { factors }\end{array}$ & \multicolumn{1}{c}{ Specification of intemal factors } & $\%$ & $\begin{array}{c}\text { Estimated annual inappropri- } \\
\text { ate days of stay }\end{array}$ \\
\hline Discharge & $\begin{array}{c}\text { - Mother of newborn has no medical } \\
\text { reason for further hospital stay }\end{array}$ & 5.7 & 646 \\
& - Other & 38.1 & 4321 \\
Therapy & - OR-scheduling & 10.4 & 1183 \\
& - Diagnostic procedures & 34.4 & 3912 \\
& - Other & 1.4 & 147 \\
Test results & - Lab results (in general) & 3.3 & 373 \\
& - CT/MRI & 1.3 & 145 \\
& - Other & 5.4 & 636 \\
\hline Total & & 100 & 11363
\end{tabular}

Estimation based on figures 1998. OR: operation room; CT: computer tomography; MRI: magnetic resonance imaging

In reducing the number of inappropriate days of stay some ethical and practical problems may occur, such as whether a patient should be discharged and readmitted

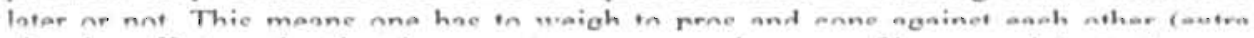
planning efforts and patient inconveniences versus decrease of inappropriate stay).

Already, several bottlenecks can be pointed out; e.g. the lack of written discharge procedures and the lack of specific process analysis and flowcharting. The lack of written discharge procedures, describing each step of the logistic process of the patient and the time involved, leads to poor control of this process. This logistic process and its time schedule should be monitored by the management. Discharge procedures should already start at the patient's admission. Specific process analysis and flowcharting can provide insight in the effective application or performance of (diagnostic) procedures. Delay in radiological diagnostic procedures (e.g. CT, MRI) could be reduced by the use of diagnostic facilities outside business hours. These hours should then be reserved for in-patient, non-urgent applications. In addition, critical evaluation of the application must be performed in order to give priority to urgent applications and to discourage unnecessary diagnostics. If needed, extra equipment could increase the capacity. The delays in test results need to be analyzed by future specific process analysis, especially the routing of the test results of the pathology lab and radiological diagnostic procedures. 
Table 5: Specification of inappropriate days of stay due to internal factors per medical department

\begin{tabular}{|c|c|c|c|c|c|}
\hline $\begin{array}{c}\text { Medical } \\
\text { department }\end{array}$ & $\begin{array}{l}\text { Internal } \\
\text { factors }\end{array}$ & $\begin{array}{l}\% \text { Within } \\
\text { med. dept. }\end{array}$ & Specification of internal factors & $\begin{array}{l}\% \text { Within } \\
\text { int. factor }\end{array}$ & $\begin{array}{l}\text { Estimated annual } \\
\text { inappropriate days } \\
\quad \text { of stay }\end{array}$ \\
\hline \multirow[t]{8}{*}{ Surgery } & Discharge & 61.5 & - No specification & 100 & 2265 \\
\hline & \multirow[t]{3}{*}{ Therapy } & \multirow[t]{3}{*}{34.6} & - OR-scheduling & 61.1 & 779 \\
\hline & & & - Diagnostic procedures & 33.2 & 424 \\
\hline & & & - Other & 5.7 & 72 \\
\hline & \multirow[t]{4}{*}{ Test results } & \multirow[t]{4}{*}{3.8} & - Pathology lab & 25.0 & 36 \\
\hline & & & - Chemical labi & 25.0 & 36 \\
\hline & & & - Other & 50.0 & 72 \\
\hline & & & Subtotal 1 & & 3681 \\
\hline \multirow{10}{*}{$\begin{array}{l}\text { Interna! } \\
\text { Medicine }\end{array}$} & Discharge & 39.6 & - No specification & 100 & 1186 \\
\hline & \multirow{3}{*}{ Therapy } & \multirow[t]{3}{*}{44.0} & - OR-scheduling & 12.5 & 177 \\
\hline & & & $=$ Diagnostic procedures & 85.0 & 1202 \\
\hline & & & - Other & 2.5 & 35 \\
\hline & \multirow[t]{6}{*}{ Test results } & \multirow[t]{6}{*}{16.5} & - Medical consultation & 36.5 & 194 \\
\hline & & & - Test results (in general) & 27.1 & 144 \\
\hline & & & - Pharmacological lab & 18.2 & 97 \\
\hline & & & - Surgical procedures & 9.1 & 48 \\
\hline & & & - Other & 9.1 & 48 \\
\hline & & & Subtotall 2 & & 3211 \\
\hline \multirow[t]{7}{*}{$\begin{array}{l}\text { Obstetrics \& } \\
\text { Gynecology }\end{array}$} & Discharge & 75.3 & $\begin{array}{l}\text { Mother of newborn has no } \\
\text { medical reason for further } \\
\text { hospital stay }\end{array}$ & 94.0 & 646 \\
\hline & \multirow[t]{3}{*}{ Therapy } & \multirow[t]{3}{*}{13.5} & - No specification & 6.0 & 46 \\
\hline & & & - Diagnostic procedures & 41.4 & 52 \\
\hline & & & - Urgency of diagnostic proce- & 41.4 & 52 \\
\hline & \multirow[t]{3}{*}{ Tesi results } & \multirow[t]{3}{*}{11.2} & dures & & \\
\hline & & & - Other & $\$ 7.2$ & 20 \\
\hline & & & - Pathology tak & 100 & 103 \\
\hline \multirow{6}{*}{ Neurology } & Discharge & 16.4 & - No specification & 100 & 498 \\
\hline & \multirow[t]{2}{*}{ Therapy } & \multirow[t]{2}{*}{75.3} & - OR-scheduling & 4.8 & 105 \\
\hline & & & Diagnostic procedures & 95.2 & 2112 \\
\hline & \multirow[t]{3}{*}{ Tesi results } & \multirow[t]{3}{*}{8.2} & - CTMRI & 59.9 & 145 \\
\hline & & & - Medical consultation & 40.1 & 97 \\
\hline & & & Subtotal 4 & & 3941 \\
\hline \multirow[t]{6}{*}{ Neuresurg. } & Discharge & 56.0 & - No specification & 100 & 342 \\
\hline & \multirow[t]{2}{*}{ Therapy } & \multirow[t]{2}{*}{40.0} & - OR-scheduling & 49.0 & 122 \\
\hline & & & - Diagnostic procedures & 51.0 & 133 \\
\hline & \multirow[t]{2}{*}{ Test results } & \multirow[t]{2}{*}{4.0} & $\cdot \mathrm{Cl}$ & 100 & 24 \\
\hline & & & Subtotal 5 & & 611 \\
\hline & & & Total & & 11363 \\
\hline
\end{tabular}

'Jstimation hased on ligures 1098 . IIIS: inappropriate hospital stay; OR: operating room; CT: computer fomography; MR : magnetic resonance imaging 
External factors cannot be directly controlled by the organization. Care supervisors are already being used to facilitate the discharge from the hospital to other care facilities. Nevertheless, inappropriate stay due to other care facilities remains a major problem. The set-up of a "low-care ward", a "care-hotel", age related geriatric services or extended home care ${ }^{31}$ might be options. These services can help the patient to recover outside a hospital setting and result in a more appropriate use of hospital resources. Some inappropriate days of stay due to internal reasons (e.g. delay in discharge) might also decrease, by the use of these facilities.

Up to 6000 days of stay could become redundant, if the inappropriate stay due to internal factors is reduced by $50 \%$. A follow-up study will be set up to analyze the current delays (waiting times for therapy, test results and / or discharge) and assess the effects of improvements actions (decrease of inappropriate stay). These effects need to be statistically significant and attributable to the improvements made. For this, a monitoring system, applying principles and techniques of statistical process control (time-series analysis), would be useful.

\section{Conclusions}

There is an increasingly interest in quality and efficiency of care. This requires simple, reliable and inexpensive systems of reviewing the appropriateness of the care provided on a regularly base. The basic features of the AEP make it, when properly modified, a usable screening tool for hospital utilization reviews. This study shows that the DAEP is a valid instrument for evaluating the appropriateness of hospital stay. The validity, reliability and user-friendliness of the instrument are adequate, and several groups of professionals can use it after a minimum of training. The DAEP can be used to evaluate the processes in and between the various departments of the hospital as well as the processes between various health care facilities. These data can enable specific quality improvement programs to be designed. Despite the differences between the Dutch and other health care systems the results do not differ substantially. $5-18$ 


\section{References}

1. Central Bureau for Statistics, Annual report (in Dutch), the Netherlands, 1999.

2. Gertman. P, Restuccia. J. The Appropriateness Evaluation Protocol: A Technique for Assessing Unnecessary Days of Hospital Care. Med Care 1981; 9:855-71.

3. Restuccia J. Appropriateness Evaluation Reviewers' Manual. In: The AEP Final Report. Springfield, VA: National Technical Information Service, 1986, Publication No. PB 87-112041.

4. Restuccia J. The evolution of Hospital Utilization Review Methods in the United States. Int J Qual Health Care 1995; 7:253-60.

5. Apolone G, Fellin G, Tampieri E, Bonanoni E et al. Appropriateness of hospital use. Report from an Italian study. Eur J Public Health 1997; 7:34-9.

6. Baré M. Prat A, Lledo L, Asenjo M, Salleras L. Appropriateness of admissions and hospitalization days in an acure-care teaching hospital. (in French) Rev Epidém et Santé Publ 1995; 43:328-36.

7. Bentes M, Gonsalves M, Santos M, Pina E. Design and development of a Utilization Review Program in Portugal. Int J Qual Health Care 1995; 7:201-12.

8. Fellin G, Apolone G, Tampieri A, et al. Appropriateness of Hospital use: an Overview of Italian Studies. Int J Qual Health Care 1995; 7:219-25.

9. Kaya S, Erdem Y, Dogrusoz S, Halici N. Reliabilitiy of a hospital utilization review method in Turkey. Int J Qual Health care 1998; 10:53-8.

10. Kaya S, Vural G, Eroglu K, Sain G et al. Reliability and validity of the Appropriateness Evaluation Protocol in Turkey. Int J Qual Health care 2000; 12:325-9

11. Lang T, Liberati A, Tampieri A, et al. A European version of the appropriateness Evaluation Protocol. Goals and Presentation. Int J of Techn Assess Health Care 1999; 15:185-97.

12. Lorenzo S, Sunol R. An overview of Spanish Studies on Appropriateness of Hospital Use. Int J Qual Health Care 1995; 7:213-8.

13. Lorenzo S, Beech R, Lang T, Santos-Eggiman B. An experience of utilization review in Europe: sequel to a BIOMED project. Int J Qual Health Care 1999; 11:13-9.

14. Lorenzo S, Lang T, Pastor R. Tampieri A et al. Reliability study of the European appropriateness evaluation protocol. Int J Qual Health care, 1999; 1 1:419-24.

15. Merom D, Shohat T, Harari G, Oren M. Green M. Factors associated with inappropriate hospitalization days in internal medicine wards in Israel: a crossnational survey. Int J Qual Health Care 1998; 10:155-62.

16. Paldi Y. Porath A, Friedman L, Mozes B. Factors Associated with Inappropriate Hospitalization in Medical Wards: a Cross-sectional Study in Two University Hospirals. Int J Qual Healih Care 1995; 7:261-5.

17. Robain M. Lang T, Fontaine A, et al. Reliability and validity of the French version of the first part of the Appropriateness Evaluation Protocol (AEPf): criteria of appropriateness of hospital days (in French). Rev Épidém et Santé Publ 1999; 47:! $39-49$.

18. Santos-Eggiman B. Paccaud F, Blanc T. Medical Appropriateness of Hospital Utilization: an Overview of the Swiss Experience. Hospital utilization reviews under field conditions: Potential and Improvements. Int J Qual Health Care 1995; $7: 227-32$.

19. Schouten H. The number of individuals needed to prove a difference between groups (in Dutch). Statistical bulletin for medicine and health care sciences. Maastricht University. 1994: 1:4-7. 
20. Schut M. Appropriate use of hospital beds. A study of the possibilities of the AEP (in Dutch). Maastricht University, 1994.

21. Esveld S. The Appropriateness Evaluation Protocol. A study of the reliability and validity of the AEP (in Dutch). Maastricht University, 1995.

22. Smeets P, Pop P, Verheggen F. Assessing the need of hospital stay (in Dutch). Medisch Contact 1997; 52: 757-61.

23. Smeets P, Verheggen F, Pop P, Panis L, Carpay J. Assessing the necessity of hospital stay by means of the appropriateness evaluation protocol: how strong is the evidence to proceed? Int J Qual Health Care 2000; 12:483.93.

24. Lavis J, Anderson G. Appropriateness in health care delivery: definitions, measurement and policy implications. Can Med Assoc 1996; 154:321-8.

25. Ash A. The Design and Analysis of Hospital Utilization Studies. Int J Qual Health Care $1995 ; 7: 245-52$.

26. Ramos-Cuadra A, Marión-Buen J, Garcia-Martin M, et al Use of the Appropriateness Evaluation Protocol: the role of medical record quality. The Effect of Completeness of Medical Records on the Determination of Appropriateness of Hospital Days. Int J Qual Health Care 1995; 7:267-75.

27. Santos-Eggiman B. Sidler M, Schopfer D, Blanc T. Comparing results of concurrent and retrospective designs in a hospital utilization review. Int $\mathrm{J}$ Qual Health Care 1997; 9::115-20.

28. Streiner D, Norman G. Health Measurement Scales: A practical guide to their development and use. Oxford: Oxford university press, 1995

29. Bouter L, van Dongen M. Epidemiological research, design and interpretation. (in Dutch) Bohn Stafleu Van Loghum, Houten, 1995 ISBN: 90-313-1879-5.

30. Hall D, Bytheway B. The blocked bed; definition of a problem. Soc Sci Med 1985; 16: 1985-91.

31. Hermans E, Anten H, Diederiks J, Philipsen H. Long-term results and substitution among 'blocked bed'-patients suffering $\mathrm{CVA}$ (in Dutch). Tijdschrift voor gezondheidswetenschappen 2000; 5: 275-9. 


\section{Appendix 1:}

\section{DAEP: LIST of Criteria for appropriate clinical stay}

\begin{tabular}{|c|c|}
\hline $\begin{array}{l}\text { 1. Threatening situations, requiring acute clinical } \\
\text { care: } \\
1.1 \text { (imminent) circulatory disorders } \\
1.2 \text { (imminent) respiratory disorders } \\
1.3 \text { (imminent) CVA / spinal cord leasion } \\
1.4 \text { inability to void or move bowel } \\
1.5 \text { other criteria, (to be specified): }\end{array}$ & $\begin{array}{l}\text { 3. Monitoring } \\
\begin{array}{l}\text { 3.1 Close medical monitoring by a nurse } \\
\geq 3 \times \text { day) under physician's orders, or ac- } \\
\text { cording to existing procedure } \\
3.2 \text { intake and output measurements. }\end{array}\end{array}$ \\
\hline \multirow{2}{*}{$\begin{array}{l}\text { 2. Care, requiring clinical control or observation: } \\
2.1 \text { scheduled for procedure the next day } \\
2.2 \text { surgical procedure in operating room } \\
2.3 \text { diagnostic procedures } \\
2.4 \text { adjustment of medication } \\
2.5 \text { adjustment of hematological disorders } \\
2.6 \text { wound and drainage care } \\
2.7 \text { patient instruction of nursing techniques }\end{array}$} & $\begin{array}{l}\text { 4. Infusions and / or medication } \\
4.1 \text { IV administration of fluids and/or nutrition } \\
4.2 \text { IV or epidural administration of medication }\end{array}$ \\
\hline & $\begin{array}{l}\text { 5. Nursing care } \\
5.1 \text { care according to existing procedure or } \\
\text { protocol } \\
5.2 \text { isolation of the patient } \\
5.3 \text { endotracheal suction }\end{array}$ \\
\hline & \\
\hline
\end{tabular}

\section{DAEP: LIST of Reasons for inappropriate clinical stay}

7. Futher clinical stay appropriate

7.1 Delay due to the late performance of tests or interventions, necessary for further treatment

7.2 Delay due to (test) results, necessary for further treatment not being available on time
8. Further clinical stay inappropriate

Due to the hospital

8.1 Ambiguous (medical) policy

8.2 delay due to discharge procedures.

Due to the patient or family

8.3 Lack of (persons for) informal care

8.4 Patient refusal to go home

Due to the environment

8.5 Primary care facilities not (yet) available

8.6 Treatment possible in outpatient or daycare setting

8.7 Patient is waiting on transfer to other care facility

9. Other reasons,

(to be specified): 
IS-AFP

\begin{tabular}{|c|c|}
\hline IIA & Medical services \\
\hline IIAI & Procedure in operating room that day \\
\hline IIA2 & $\begin{array}{l}\text { Scheduled for procedure in } \mathrm{OR} \text { the next day. } \\
\text { requiring extraordinary preoperative consulta- } \\
\text { tion }\end{array}$ \\
\hline IIA3 & Cardiac catheterization that day \\
\hline IIA4 & Angiography that day \\
\hline IIA5 & Biopsy of internal organ that day \\
\hline IIA6 & Invasive CNS diagnostic procedure that day \\
\hline IIA7 & $\begin{array}{l}\text { Any test requiring dietary control, for the } \\
\text { duration of the diet }\end{array}$ \\
\hline
\end{tabular}

IIA8 New or experimental treatment, requiring. frequent dose adjustments under direet medical supervision

IIA9 Condition requiring close medical monitoring by a doctor at least 3 times daily

IIA 10 Major invasive procedure within the past 24 hours

\begin{tabular}{l|l} 
IIE & Nursing / life-support service \\
\hline IIB11 & $\begin{array}{l}\text { Respiratory care: intermittent or continuous } \\
\text { respirator use and/or inhalation therapy }\end{array}$
\end{tabular}

IIB 12. Parenteral therapy: intermittent or continuous IV fluid with any supplementation

IIB 13 Continuous monitoring of vital signs, at least. every $30 \mathrm{~min}$., for at !east 4 hours

IIBI4 Intramuscular and subcutaneous injections at least twice daily

IIBI5 Intake and output measurements

IIB16 Major surgical wound and drainage care

IIB 17. Close medical monitoring by a nurse at leas? three times daily under physician's orders

\section{DAEP}

Procedure in operating room that day, requiring clinical control or observation

Scheduled for procedure the next day, requiring clinical control or observation

Cardiac catheterization is not considered as an DAEP-criterion per se

Angiography that day is not considered as an DAEP-criterion per se

Biopsy of internal organ that day is not considered as an DAEP-criterion per se

Diagnostic procedures, requiring clinical control or observation

Amy test requiring dietary control, for the duration of the diet is not considered as an $D A E P$-criterion per se

Adjustment of medication, requiring clinical control or observation

\section{Combined with IIB 17}

Care according existing procedure or protocol

Respiratory care: intermittent or contimuous respirator use andor inhalation therapy is not considered as an DAEP-criterion per se

IV administration of fluids and/or nutrition

Combined with IIB 17

IV or epidural administration of medication

Intake and output measurements

Wound and drainage care, requiring clinical control or observation

Close medical monitoring by a nurse at least three times daily under physician's orders, or according to existing procedure or protocol Patient instruction of nursing techniques

Isolation of the patient

Endotracheal suction 
Appendix 2 Difference between US-AEP and DAEP (contimued)

\section{US-AET}

\begin{tabular}{|c|c|c|}
\hline IIIC & Patient condition & \\
\hline IIC18 & $\begin{array}{l}\text { Inability to void or move bowel (past } 24 \text { hours) } \\
\text { not attributable to underlying neurological } \\
\text { disorder }\end{array}$ & $\begin{array}{l}\text { Inability to void or move bowel, requiring acute } \\
\text { clinical care }\end{array}$ \\
\hline IICI9 & Transfusion due to blood loss & Combined with IIC20 \\
\hline IIC 20 & $\begin{array}{l}\text { Ventricular fibrillation or ECG evidence of } \\
\text { acute ischaemia, as stated in progress note or in, } \\
\text { ECG report }\end{array}$ & $\begin{array}{l}\text { Circulatory disorders, requiring acute clinical } \\
\text { care }\end{array}$ \\
\hline IIC2I & $\begin{array}{l}\text { Fever of at least } 101^{\circ} \mathrm{F} \text { rectally if the patient } \\
\text { was admitted for reasons other than fever }\end{array}$ & $\begin{array}{l}\text { Fever of at least } 10 I^{\circ} \text { F rectally if the patient } \\
\text { was admitted for reasons other than fever is not } \\
\text { considered as an DAEP-criterion per se }\end{array}$ \\
\hline IIC 22 & Coma, unresponsiveness for at least 1 hour & Coma, unresponsiveness for at least I hour \\
\hline $\mathrm{IIC} 23$ & $\begin{array}{l}\text { Acute confusional state, not due to alcohol } \\
\text { redrawal }\end{array}$ & $\begin{array}{l}\text { Acute confusional state, not due to alcohol } \\
\text { redrawal is not considered as an DAEP-crite- } \\
\text { rion per se }\end{array}$ \\
\hline IIC24 & Acute hematological disorders. (...) & $\begin{array}{l}\text { Adjustment of hematological disorders, requir- } \\
\text { ing clinical control or observation }\end{array}$ \\
\hline $11 \mathrm{C} 25$ & Progressive acute neurological difficulties & $\begin{array}{l}\text { Imminent CVA / spinal cord lesion, requiring } \\
\text { acute clinical care }\end{array}$ \\
\hline & & Other reasons, requiring acute clinical care: \\
\hline
\end{tabular}



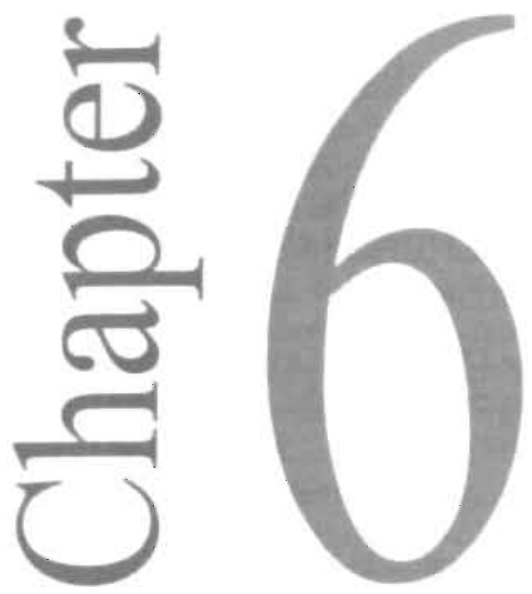

How appropriate is inappropriate stay?

Int I Health Care Qual Assurance 2004: 17 (2) 


\begin{abstract}
Background.

Extended day care (EDC) is a one-day admission spending one night in hospital. Many EDC patients do not need hospital care over night, so probably they could be transferred to a day surgery setting, resulting in decreased costs and increased efficiency.
\end{abstract}

\title{
Objectives.
}

To assess the appropriate length of extended day care (ALED) and a possible transfer to day surgery. ALED was defined as the time between the start of the surgical procedure and the final moment appropriate hospital care was provided.

Results.

About $80 \%$ of the patients could possibly have been treated in day surgery. The other patients could not be transferred, because a prolonged ALED.

\section{Conclusions.}

With the implementation of new policies on admission to and discharge from the hospital and the use of altered types of operation room scheduling or patient logistics the transfer of most EDC patients to day surgery would be possible.

Keywords: Dutch Appropriateness Evaluation Protocol, extended day care, appropriate length of stay. 


\section{Introduction}

In the Netherlands almost $11 \%$ of the Gross National Product is being spend on health care (in 2001: 46 billion Euros). 'One third of these costs is related to intramural care. Improving the efficiency of hospital services could contribute to cost containment. This could be realized in several ways: e.g.: to avoid inappropriate hospital stay or to decrease the number of hospital admissions by increasing the number of patients in day surgery. Although in recent years a continuous shift from in-patient care to day surgery and to outpatient care occurred in the Dutch health care system, this shift can still be optimalized even more. And since day surgery is less expensive for several care processes, while patient outcomes are proven to be good, the possibilities of a further shift should be examined.

From a financial point of view, there are three categories of patients in the Dutch healthcare system:

- Outpatients, needing less than two hours of follow up after consultation / treatment;

- Day surgery patients, needing at least two and at the most 24 hours of follow-up:

- In-patients admitted to a hospital for a follow-up of more than 24 hours.

Patients for day surgery are admitted, attended to and discharged within regular business hours. If patients need hospital care outside these hours, but less than 24 hours, they are admitted to the hospital as extended day care (EDC) and financially charged as a regular admission. So, the expression 'extended day care' is delusive, it is in fact a hospital admission. EDC patients arrive at a shori-stay unit in the moming and surgery is performed early in the afternoon. (A short stay unit is a specific nursing ward where

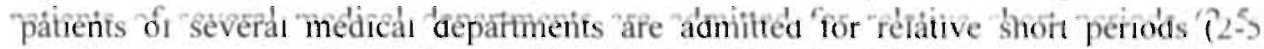
days) and consequently discharged from the hospital. For this aim, care and treatment procedures are standardized to an optimum.) The next moming the EDC patienis are discharged from the short stay unit, even though they might not have needed any care or surveillance during the night. If these EDC patients could be treated in day surgery, costs would decrease, while capacity (in terns of available hospital beds) would increase. To obtain this shift, the appropriate length of EDC should be assessed in order to formulate new policies on hospital admission and discharge.

\section{Previous research}

Previous studies at the university hospital of Maastricht ${ }^{2-6}$ evaluated the inappropriate hospital stay and ways to measure it in a valid and reliable way, resulting in the Dutch Appropriateness Evaluation Protocol (DAEP). This instrument consists of 19 generic (diagnosis-independent) and explicit (care related) criteria for appropriate hospital stay and a list of reasons for inappropriate stay. These studies showed that over $20 \%$ of the regular hospital stay was inappropriate according to the DAEP and that almost half of this inappropriate stay was due to internal hospital procedures, such as delays in discharge or therapeutic / diagnostic procedures. Several international studies ${ }^{7.20}$ also showed significant proportions of hospital stay to be inappropriate elsewhere. Most of these studies however focused on the assessment of hospital admission or stay. Few studies were found assessing the appropriateness within a day care setting. 


\section{Objectives}

In order to assess the specific nature of EDC a pilot study was initiated exploring possibilities to shift these patients from an in-patient to a day surgery setting. Therefore the aims of this study were:

- To assess the appropriate length of hospital care of EDC patients.

- To assess opportunities for a possible transfer of EDC patients from an in-patient to a day surgery setting.

Only the appropriateness of the setting in which the care was provided has been assessed in this study, not the appropriateness of care itself or the appropriateness of the patient's admission.

\section{Methods}

\section{Setting}

This study was conducted at one short-stay ward of the university hospital of Maastricht, a 715-bed, teaching hospital, located in the southeastern part of the Netherlands. Yearly, approximately 22,000 patients are admitted to this hospital and about 200,000 days of stay are generated with an average length of stay of 8.9 days (nationwide 9.2 days,). About 1,000 days of stay (i.e. $0.5 \%$ of the days mentioned above) were extended daycare. Beside these 1,000 patients treated in EDC approximately 6,000 patients are treated in day surgery annually.

\section{Assessment of the appropriate length of hospital care in extended daycare (EDC)}

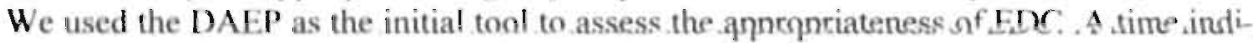
cation was added to the DAEP to indicate the moment of surgery and the moment of final appropriate hospital care. The DAEP was added to the patients file and scored concurrently by registered nurses involved in the care of these patients. Appropriate hospital care was defined as: 'Inpatient care, requiring continuous and active medical, mursing or paramedical treatment, which under existing legislation can not be provided through extramural care, day care or outpatient care., 5 while appropriate length of EDC (ALED) was defined as: 'The time between the start of the surgical procedure and the moment that hospital care was still appropriate'.

\section{Study sampling and data collection}

The study was set up as a prospective cohort study. Data was collected at the short-stay ward ( 26 beds) where also EDC patients (one day) were admitted beside regular shortstay patients (2-5 days of hospital stay). During a four-month period (April - July 2001 ). the hospital stay of 218 EDC patients was assessed by registered nurses $(n=12)$. These nurses assessed the appropriateness of the hospital stay every hour using the DAEP, evaluating the required care and the moment of care giving. Reviewed data included the date of treatment, the patient registration number, age, sex, type and time of surgery, the individual DAEP score and time of surgery and appropriateness of hospital care. 


\section{Statistical analysis}

Descriptive analysis was applied, to the data concerning the appropriateness of EDC. During the study period, no correlated measurements in the same patient occurred. We used the student's t-test to test the mean age between groups. The Pearson Chi-square test was used to test the significance of differences between groups. All data analyses were performed using the SPSS-PC software package, version 10.0.

\section{Results}

During a four-month period the hospital stay of 218 EDC patients was assessed. The data on the stay of 10 patients was excluded from further analysis. (In two patients $(0.96 \%)$ complications occurred, requiring prolonged hospital admission, while data on eight patients was incomplete.) Eighty-nine patients were male $(42.8 \%)$; the mean age of all the patients was 45.6 years (SD 19.8), with a range between 11 to 97 years.

\section{Appropriate length of EDC}

The start of the required surgical procedures and the corresponding ALED is shown in figure 1. Procedures with the longest ALED appear to be scheduled earlier on the day. For instance: if the surgical procedure starts at 12.00 AM an average ALED of 8 hours is required, suggesting EDC patients do not need any further hospital care after 08.30 PM. However, 42 EDC patients (20.2\%) still needed hospital care after 10.00 PM (table 1 \& fig. 2). Especially patients for orthopedic and plastic surgery procedures needed longer time to recover. These patients had an average ALED of over 12 hours $(722$ min., sd: $105 \mathrm{~min})$. About one quarter $(24 \%)$ of the EDC patients $(\mathrm{n}=50)$ did not need any further hospital care after 8.00 PM. These patients had an ALED of 274 minutes (4.6 hours, sd $54 \mathrm{~min}$ ). The remaining 116 patients $(55.8 \%$ ) did not need further hospital care after 10.00 PM. They had an ALED of 444 minutes $(7.4$ hours, sd: 71 min.).

Fig. 1: Start surgical procedure \& corresponding ALED

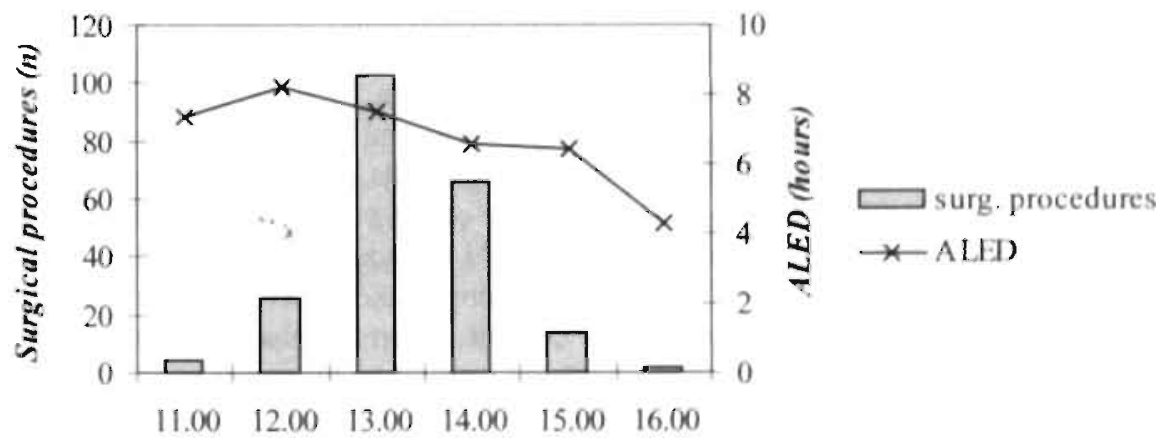

start surgical procedure

\section{Factors determining length of stay}

Although the ALED was not statistically significantly related to patient characteristics, medical specialism or moment of surgery, some types of surgery show a particular length of stay. Most procedures in Ear-Nose-Throat medicine (ENT) have a relative short ALED. Adenotonsillectomy e.g. $(n=26)$ has an ALED of 6.9 hours $(417 \mathrm{~min}$., SD 142). On the other hand, several procedures in orthopaedic surgery (e.g. removal of 
fixation devices) and plastic surgery (mamma augmentation or reduction) require a longer hospital stay (fig. 2). The procedures for which a longer stay was required were: breast augmentation or reduction, eyelid reconstruction, relaxation of scar tissue, removal of internal fixation devices and in- / excision of joint structures (table 2). These procedures have an ALED of over 12 hours. Table 3 shows that these 'long' procedures are specifically related to the intravenous administration of fluids and / or medication and monitoring of the patient. This relation was proven statistically significant (p: .016).

Table 1: Appropriareness of hospital stay vs. medical specialism

\section{END OF APPROPRIATE HOSPITAL CARE}

\begin{tabular}{lcccc} 
Specialism $(\mathrm{n})$ & before $8.00 \mathrm{PM}$ & $8.00-10.00 \mathrm{PM}$ & after $! 0.00 \mathrm{PM}$ & Total \\
\hline General. Surgery & 11 & 33 & 7 & $51(24.5 \%)$ \\
Ear-Nose-Throat & 15 & 26 & 4 & $45(21.7 \%)$ \\
Orthopedic Surgery & 10 & 22 & 13 & $45(21.7 \%)$ \\
Plastic Surgery & 8 & 18 & 13 & $39(18.7 \%)$ \\
Urology & 5 & 12 & 2 & $19(9.1 \%)$ \\
Other & 1 & 5 & 3 & $9(4.3 \%)$ \\
\hline Total & $50(24.0 \%)$ & $116(55.8 \%)$ & $42(20.2 \%)$ & 208
\end{tabular}

'Appropriate length of extended day care

\section{Discussion}

EDC is a pragmatic example of recent logistic changes in surgical care processes, resulting in reduced hospital stay. This study indicates that 7 to 10 hours after specific surgical procedures in EDC, no further hospital stay is required for these patients. This suggests than in total approximately $80 \%$ of the EDC patients could be transferred to a day surgery setting. This would increase the efficiency of the current hospital facilities (day surgery) and the hospital stay of EDC patients could be reduced significantly.

Within the current setting. minor changes in planning and scheduling would improve patient logistics. If, for example, within the current system of planning and scheduling, regular treatment hours (i.e. 9.00 AM to 5.00 PM) would be extended by three hours, or if within these regular hours surgical procedures would start three hours earlier, $25 \%$ of these $\mathrm{EDC}$ patients could be discharged at the same day. This extension together with an earlier planning of surgical procedures would increase the proportion of EDC patients discharged at the same day to $80 \%$.

However, several patient groups need longer time to recover adequately or they need extra observation or monitoring, in order to leave the hospital with a minimum of risk. 24 Also possible complications that might occur after surgery (about $1 \%$ in this study) have to be taken into account. These complications are often related to existing comorbidity and usually occur in the first 24 hours after surgery. "Therefore, it is evident to continue the treatment of these EDC patients on in-patient basis in order to leave the 
hospital with a minimum of risk. Although it is not expected that an early discharge will lead to more complications or discomfort for the patient, supplementary facilities, such as the back up of home care, should be available if EDC patients would be transferred to a day surgery setting. If any complications after discharge might occur in the home situation, proper actions could be taken with these precautions. ${ }^{20}$ Improving EDC seems to be a matter of resourcing and logistics. ${ }^{27}$ What kind of surgery needs be planned at what time, which operational policies and patient logistics have to be altered?

Fig. 2: End of appropriate hospital care (in \%)

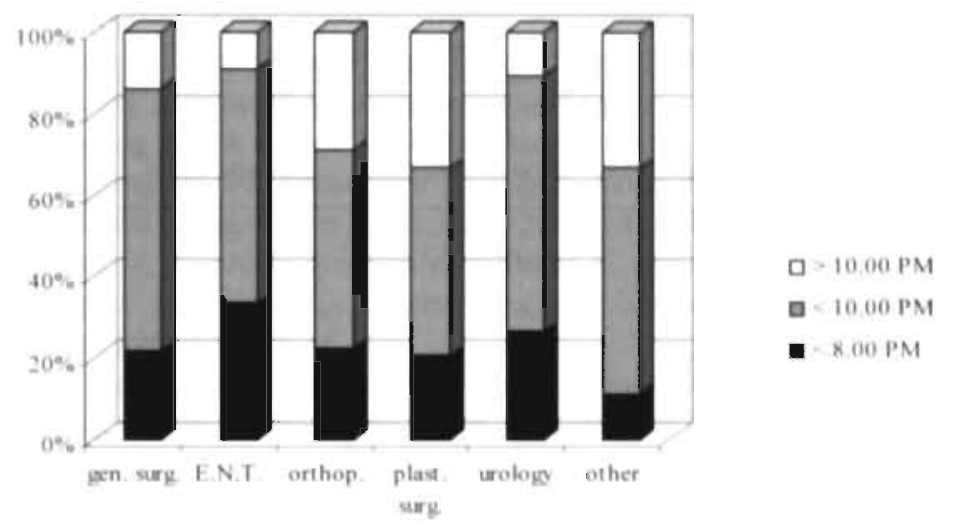

specialisms

For feasibility of these options, several possibilities have to be considered in more detail. First, by transferring an EDC patient to day surgery, this patient would 'occupy' a bed for the whole day. In the current system of patient logistics in day surgery two to three patients can use the same bed facility in one day. So a cost-benefit analysis has to be made in order to select the most efficient patient logistics; one EDC patient or several day surgery patients. ${ }^{28}$ Secondly, existing comorbidity might require extra observation in a hospital setting to eliminate possible complications. Thirdiy, if EDC patients are transferred to day surgery the workload on the short-stay ward might increase. Since the patient with a less need for care will be transferred, patient with a higher need for nursing care will remain on the ward. ${ }^{29}$

Table 2: 'Long stay' within extended day care

\begin{tabular}{lll}
\hline \multicolumn{1}{c}{ Surgical procedure } & Medical specialty & ALED in min. (SD) \\
\hline Mamma augmentation / reduction & Plastic surgery & $747(41)$ \\
Eyelid reconstruction & Plastic surgery & $860(200)$ \\
Relaxation of scar tissue & Plastic surgery & $750(129)$ \\
Removal of internal fixation device & Orthopedic surgery & $776(92)$ \\
In- / excision of joint structures & Orthopedic surgery & $648(43)$
\end{tabular}




\section{Conclusion}

The DAEP can be used to assess the appropriate length of stay. This study demonstrates that, with the current services, there is evidence that the majority of EDC patients can be transferred to a regular day surgery setting. However, this requires a redesign of the current planning of surgical procedures. Beside that, follow-up studies are needed to determine specific patient groups for EDC to be treated in a regular day surgery setting, next to additional research on the implications in workload of the nursing staff. And supplementary, adequate home care or back-up facilities in case of any complications should also be available.

Table 3: Nursing care during EDC

APPROPRIATE HOSPITAL CARE

\begin{tabular}{|c|c|c|c|c|}
\hline Nursing care & before 8.00 PM & $8.00-10.00 \mathrm{PM}$ & after 10.00 PM. & Total \\
\hline Observation & & 2 & 6 & 8 \\
\hline Infusion/medication & 11 & 17 & 19 & 47 \\
\hline Monitoring & 39 & 82 & 14 & 135 \\
\hline Specific nursing care & & 15 & 3 & 18 \\
\hline Total & 52 & 116 & 40 & 208 \\
\hline
\end{tabular}

Pearsun Chi-square 15.639, df 6, p: 016

\section{Acknowledgements}

We want to thank A. Pfaff, MD for pointing out the possibility of quality improvement in the treatment and logistics of EDC patients. 


\section{References}

1. Statistics Netherlands, Anmual report (in Dutch), the Netherlands, 2001. http://statline.cbs.nl/StatWeb/start.asp?LA=nl\&DM=SLNL\&lp=Search/Search.

2. Smeets P, Pop P. Verheggen F. Assessing the appropriateness of hospital stay: the possibilities of the appropriateness evaluation protocol (in Dutch). Med Contact 1997: 52: 757-60.

3. Smeets P, Pop P. Verheggen F. Assessing the appropriateness of hospital stay: features of quality assurance and utilization review tools (in Dutch). Med Contact 1997: 52: 760-61.

4. Smeets P, Verheggen F, Pop P, Panis L, Carpay J. Assessing the necessity of hospital stay by means of the appropriateness evaluation protocol: how strong is the evidence to proceed? Int J Qual Health Care, 2000; 12: 483-93.

5. Panis L, Pop P, Verheggen F. To stay or not to stay. The assessment of appropriate hospital stay, a Dutch report. Int J Qual Health Care, 2002; 14: 55-67.

6. Panis L, Gooskens M, Verheggen F, Pop P, Prins M. Predictors of inappropriate hospital stay: a clinical case study. Int J Quality Health Care 2003; 15:57-65.

7. Baré M, Prat A. Lledo L, Asenjo M, Salleras L. Appropriateness of admissions and hospitalization days in an acute-care teaching hospital. (in French) Rev Epidém et Santé Publ 1995; 43: 328-36.

8. Bentes M, Gonsalves M, Santos M, Pina E. Design and development of a Utilization Review Program in Portugal. Int J Qual Health Care 1995; 7: 201-12.

9. Black N. Appropriateness of medical care in Europe; a commentary. Int J Qual Health Care, 1994: 6: 231-2.

10. Fellin G. Apolone G. Tampieri A, et al. Appropriateness of Hospital use: an Overview of Italian Studies. Int J Qual Health Care 1995; 7: 219-25.

11. Kaya S, Erdem Y, Dogrusoz S. Halici N. Reliabilitiy of a hospital utilization review method in Turkey: Int J Qual Health care 1998; 10: 53-8.

12. Kossovsky M, Chopard P. Bolla F et al. Evaluation of quality improvement interventions so reduce inappropriate hospital use. Int J Qual Health care 2002; 3:227-32.

13. Lang T. Liberati A, Tampieri A, et al. A European version of the appropriateness Evaluation Protocol. Goals and Presentation. Int J of Technology Assessment in Health Care 1999; 15: 185-97.

14. Lorenzo S, Suñol R. An overview of Spanish Studies on Appropriateness of Hospital Use. Int J Qual Health Care 1995; 7: 213-8.

15. Lorenzo S, Beech R, Lang T, Santos-Eggiman B. An experience of utilization review in Europe: sequel to a BIOMED project. Int J Qual Health Care 1999; 11:13-9.

16. McDonagh M, Smith D, Goddard M. Measuring appropriate use of acute beds. A systematic review of methods and results. Health Policy 2000; 53:157-84.

17. Merom D, Shohat T. Harari G, Oren M, Green M. Factors associated with inappropriate hospitalization days in internal medicine wards in Israel: a cross-national survey. Int J Qual Health Care 1998; 10: 155-62.

18. Paldi Y, Porath A. Friedman L. Mozes B. Factors Associated with Inappropriate Hospitalization in Medical Wards: a Cross-sectional Study in Two University Hospitals. Int J Qual Health Care 1995; 7:261-5.

19. Robain M, Lang T, Fontaine A, et al. Reliability and validity of the French version of the first part of the Appropriateness Evaluation Protocol (AEPf): criteria of appropriateness of hospital days (in French). Rev Épidém et Santé Publ 1999; 47: 139. 49. 
20. Santos-Eggiman B, Paccaud F, Blanc T. Medical Appropriateness of Hospital Utilization: an Overview of the Swiss Experience. Hospital utilization reviews under field conditions: Potential and Improvements. Int J Qual Health Care 1995; 7: 227-32.

21. Lewis C, Wahl J, Yust K, Kaplan S. Same-day surgery managed care: monitoring patient and system variances. J Perianes Nurs 2000; 15:12-9.

22. Junger A, Klasen J, Benson M, Sciuk G, Hartmann B, Sticher J, Hempelmann G. Factors determining length of stay of surgical day-case patients. Eur $\mathbf{J}$ Anaesth. 2001; 18:314-21.

23. Bianco A, Foresta M, Greco M, Teti V, Angelillo I. Appropriate and inappropriate use of day-hospital care in Italy. Public-Health; 2002 Nov; 1 16: 368-73.

24. Clarke A. Length of hospital stay and its relationship to quality of care. Qual Saf Health Care 2002; 11:209-10.

25. Clarke A, Rosen R. Length of stay. How short should hospital care be? Eur J Public Health 2001; 11:166-70.

26. Halfon P, Eggli Y, van Melle G, Chevalier J, Wasserfallen J, Burnand B. Measuring potentially avoidable hospital readmissions. J Clin Epidemiology 2002; 55:573-87

27. Fox N. Power, control and resistance in the timing of health and care. Soc Science \& Med 1999; 48: 1307-19.

28. Rozich J. Resar R. Using a unit assessment tool to optimize patient flow and staffing in a community hospital. J Quality Impr. 2002; 1:31-41.

29. Cooper R, Aiken L. Human inputs: the health care workforce and medical markets. J Health Politics, Policy \& Law 2001: 26:925-38. 

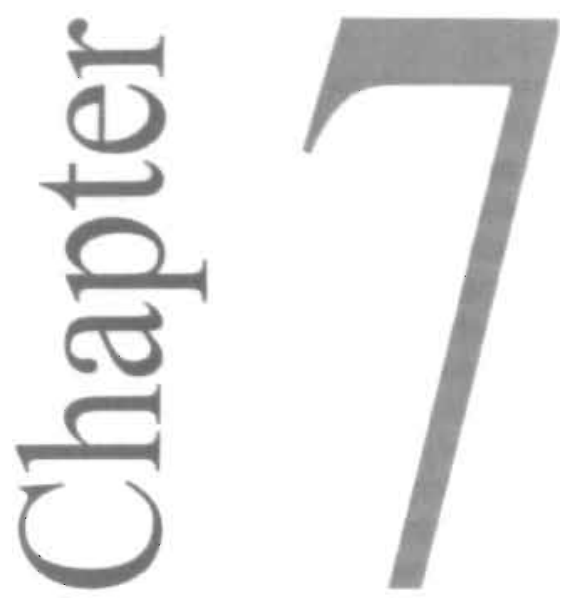

\section{Identifying inappropriate hospital stay}

Accepted as: Identifime imappropriate hospital stan in patients with venous thromboembolism

Panis I. Kolhach D. Hamulyak, K. Prins M

Eur. I. Ini Med. 


\begin{abstract}
Background.

Traditionally, venous thromboembolism was treated in a hospital setting. Nowadays, low molecular weight heparin preparations allow most deep venous thrombosis (DVT) patients to benefit from home therapy. The objective of this study was to evaluate if the previous treatment of deep venous thrombosis in a hospital setting was appropriate in the context of modern opinion, using the Dutch Appropriateness Evaluation Protocol (DAEP). If so, the DAEP could be used to assess the appropriateness of the present hospital stay of other patient groups.
\end{abstract}

\title{
Methods.
}

A retrospective research of patients treated during $1995-1998$ for DVT or pulmonary embolism (PE) during 1995 - 1998 (before implementation of ambulatory treatment) was conducted, assessing the appropriateness of the patient's stay using the Dutch Appropriateness Protocol (DAEP).

\section{Results.}

Only $27.1 \%$ of the treatment for DVT was found to be appropriate in a hospital setting and related to specific hospital care. The inappropriate stay was mostly related to delays in diagnostic and discharge procedures. Of the patients with PE, $50.2 \%$ needed hospital stay. This proportion was statistically significantly higher than in DVT patients ( $\mathrm{p}$ : < .001 ). The extent of the DVT was not related to the length of bed rest prescribed.

\section{Conclusions.}

The DAEP was able to identify inappropriate hospital stay in the past within DVT patient. Further exploration for the potential of the DAEP to identify patient groups who possibly could be treated at home is warranted.

Keywords: deep venous thrombosis, bed rest, appropriate hospital stay, length of stay. 


\section{Introduction}

The annual incidence of deep venous thrombosis (DVT) and / or pulmonary embolism (PE) is 2-3 per 1000 inhabitants. 'Traditionally, patients with venous thromboembolism (VTE) were treated with intravenous heparin and strict bed rest, followed by oral anticoagulants. Hospital admission was recommended to facilitate treatment with intravenous (IV) heparin for at least five days and was frequently associated with bed rest. The approval of low molecular weight heparin preparations (LMWH) for the treatment of DVT has revolutionized the management of this disease. In the past DVT patients were discharged from the hospital only when appropriate anticoagulant levels were reaches with oral medication after initial IV heparin. Several meta-analyses involving randomised controlled studies have shown that LMWH administered subcutaneously is as effective and safe as unfractionated heparin given intravenously. ${ }^{2}$ Laboratory monitoring is not necessary and body weight adjusted doses of LMWH can be injected subcutaneously which allows home therapy of DVT for a majority of patients. ${ }^{3.4}$ Nowadays, even early hospital discharge in patients with pulmonary embolism (PE) treated with LMWH is possible. ${ }^{5}$ Today, there is a tendency to reduce the length of hospital stay or even avoid hospital admissions, mostly due to budget restrictions and for efficiency purposes. This tendency has led to the development of several tools to assess the appropriateness of hospital admissions and / or stay and to provide management information to control these processes. ${ }^{6}$ Appropriate stay is considered to be: up to standard, effective, efficient and tailored to the patients' actual needs. ${ }^{7}$ In other words: inappropriate hospital stay is neither effective (it serves no clinical purpose) nor efficient (resources cannot be used optimally). Tools to assess appropriateness of hospital stay could also be used in order to systematically search for patient groups for whom hospital stay could be avoided.

Since the management of DVT has been proven as effective and safe in an ambulatory setting as in a hospital setting, the hospital stay of most DVT patients in the past can be considered inappropriate in the context of modern opinion. If the past 'inappropriate' hospital stay could be identified by the use of a 'modern' tool, this would be an indication that this tool could indeed be used to assess the appropriateness of present hospital stay of other patient groups. Hence, the objective of this study was to evaluate if in the past the treatment of deep venous thrombosis in a hospital setting was appropriate in the context of modern opinion, using the Dutch Appropriateness Evaluation Protocol (DAEP). Therefore, in this study patients admitted for PE were included as a comparative group. 


\section{Methods}

\section{Setting}

This study was conducted at the university hospital of Maastricht. This 715-bed teaching hospital is located in the south eastern part of the Netherlands. In 2001 this hospital admitted about 22,000 patients and generated about 200,000 days of stay with an average length of stay of 9 days. The treatment protocol of both DVT and PE consisted until 1996 of intravenous unfractionated heparin and directly starting of oral anticoagulantia. Subsequently the DVT treatment protocol changed to LMWH and oral anticoagulantia, one-day bed rest, and ambulant compression therapy. PE continued to be treated intravenously with unfractionated heparin and bed rest. At home treatment of deep venous thrombosis was introduced during 1999.

\section{Assessment of the appropriateness of bed rest in the treatment of DVT}

We used the Dutch Appropriateness Evaluation Protocol (DAEP) to assess the appropriateness of bed rest in the treatment of DVT. Appropriate hospital stay was defined as: "Hospital stay, requiring continuous and active medical, nursing or paramedical treatment, which could not be provided through extramural care, day care or outpatient care with regard to existing legislation". The DAEP is a modification of the US - medicaladult Appropriateness Evaluation Protocol ${ }^{8}$ for use in the Dutch health care system and was proven to be valid, reliable and easy to use. ${ }^{9}$ This instrument assesses the appropriateness of the setting in which care is provided, not the appropriateness of care itself. ${ }^{10}$ The DAEP a criteria-based decision support tool to determine the necessity of hospital stay basod on sots of Eenoric (diagnusis-intependenc) and explic ic (care ielated) uriteria. It consists of 19 criteria for appropriate stay, related to medical and nursing services and a list of reasons for inappropriate hospital stay. If one or more of these criteria can be met that day of stay is considered to be "appropriate" in a hospital setting, if not, the list of reasons has to be filled out, specifying the reason for inappropriate stay. Although until 1996 the IV administration of heparin was a criterion for appropriate stay, in this. study the IV administration of heparin was not scored for as such a criterion. Otherwise, considering that ambulatory therapy is a good alternative for treatment with IV heparin, it was not possible to differentiate between appropriate hospital stay in the past and in the present. Bed rest was defined as "compulsory stay in bed all day, with permission to go to the bathroom".

\section{Study sampling and data collection}

The study was set up as a retrospective cohort study. The written and electronic medical charts from patients admitted for venous thromboembolism (VTE) during the period from January 1995 to January 1999 were reviewed, blinded for the researchers. If a patient was admitted several times in this period, each hospital stay of this patient was assessed separately. Periods of hospital stay that concerned VTE other than in leg and or lung were excluded for further review. The following data were extracted from the hospital information system and patient charts: age, gender, date of admission and discharge and data on objective testing for DVT and PE. Documentation was done by ultrasonography or venography in DVT or ventilation lung scanning in PE. In addition, it was assessed if VTE was the main reason for hospital admission (primary diagnosis) or not (secondary diagnosis). 
All days of hospital stay during treatment of VTE were then evaluated using the DAEP to assess the appropriateness of the patient's stay for treatment of VTE in a hospital setting.

\section{Statistical analysis}

The analysis was restricted to patients with objectively documented diagnosis of DVT or PE. Periods of stay were considered to be related to. DVT or PE with or without DVT. Based on the available medical data, patients were divided into two groups; DVT positive (without PE) and PE positive with our without diagnosed DVT. Data on DVT and/or PE negative patients were excluded from the analysis. Descriptive statistics were calculated for the separate groups. We used the Students t-test to test the difference in means between groups if data were normally distributed, while the Mann Whitney Utest was used if data were not normally distributed. The Pearson Chi-square test was used to test the significance of differences in frequencies between groups. All data analyses were performed using the SPSS-PC software package, version 10.0 .

\section{Results}

Between January 1995 and January 1999, a total 639 admissions ( 596 patients) coded as venous thromboembolism (VTE) occurred. Data on 29 admissions (27 patients) were not available and 51 admissions ( 50 patients) for other than leg vein thrombosis or pulmonary embolism were not assessed. Hence, information on 559 admissions (519 patients) for positive DVT and/or PE was available for analysis. The diagnosis of VTE was objectively documented by ultrasonography and / or perfusion ventilation lung scanning in 511 admissions.

The clinical characteristics of these patients are shown in table 1. Data showed the mean age to be statistically significantly higher in PE patients. There was no difference found in gender. Overall, in $60 \%$ of the patients DVT and/or PE was the primary diagnosis. The median length of hospital stay was rather high in both groups (DVT: 9 days, PE: 12 days). This length of stay was mainly due to patients with secondary DVT or PE (median: 19 days), while in all patients with a primary diagnosis the median length of stay was only 9 days.

Table 1: clinical characteristics

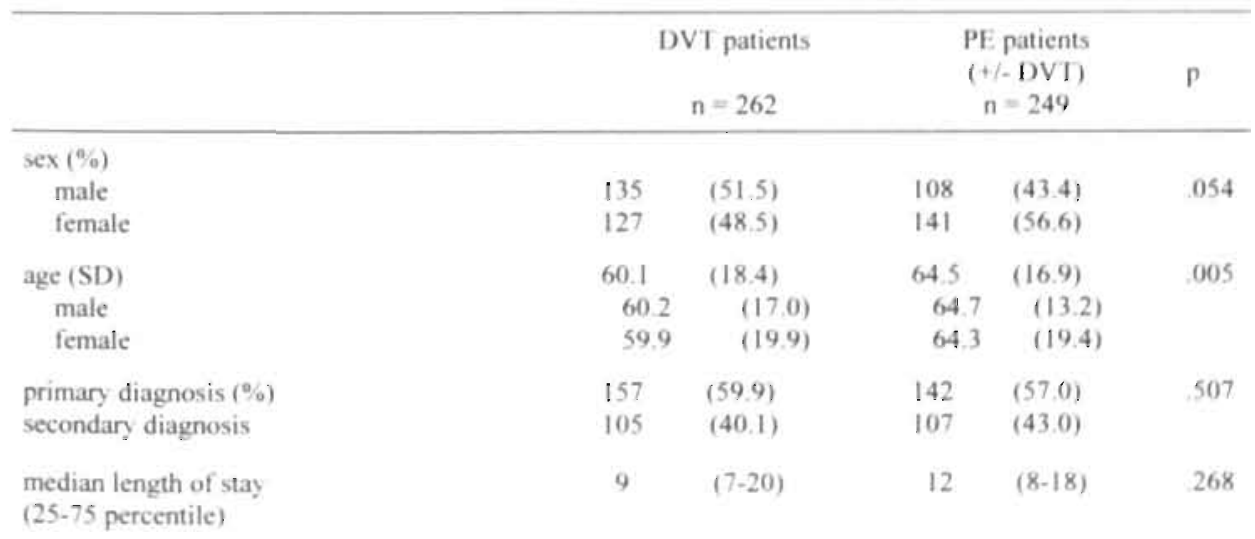


Since co-morbidities can influence the length of hospital stay and bed rest, only data on the group with a primary diagnosis were used for further analysis. The median length of stay of the patients with a primary DVT or PE diagnosis was 8 - 10 days (table 2). During this stay the patients were actively treated for DVT and / or PE for 4 or 7 days. Data collected on the height of the DVT did not show any difference between level of thrombosis and the length of bed rest prescribed.

\section{Appropriateness of stay}

As said before, only data on patients with a primary diagnosis were assessed. The length of stay and the type and duration of the treatment for DVT and PE are given in table 2. The treatment consisted of intravenous (IV) heparin, subcutaneous low molecular weighted heparin (LMWH) and bed rest, IV heparin combined with bed rest or only LMWH. Of the 157 patients with a primary DVT, only $28.0 \%(\mathrm{n}=44)$ met one or more of the DAEP criteria during the period of treatment, indicating this stay to be 'appropriate". This stay was mainly related to care, requiring specific observations $(48.3 \%)$ or the administration of medication / infusion ( $36.6 \%$ ) excluding the IV administration of heparin (Table 3). In PE patients $(n=142)$, the proportion of 'appropriate stay' was $51.4 \%$. This proportion was statistically significantly higher than in patients admitted for DVT ( $p:<.001)$. In 73 patients with PE, the frequency of (imminent) threatening situations was higher than in the 44 DVT-patients ( $23.2 \%$ vs. $7.8 \%$ ). The inappropriate stay in both groups was mainly related to delays in internal hospital and discharge procedures ( $88.9 \%$ vs. $83.2 \%$ ). About one quarter of this inappropriate stay was related to delays in the application or the logistics of diagnostic test performance. Within the DVT group, not clear defined medical policy caused about $15 \%$ of the inappropriate stay. The unavailability of other care facilities (e.g. homes for the elderly, rehabilitation homes, primary and / or home care) resulted in only a small proportion of inappropriate stay in both groups ( 7.4 vs. $5.6 \%)$.

Table 2: Treatment of primary DVT / PE

\begin{tabular}{|c|c|c|c|c|}
\hline & \multicolumn{2}{|c|}{ Primary DVT } & \multicolumn{2}{|c|}{ Primary PE } \\
\hline & Days & Patients & Days & Patients \\
\hline $\begin{array}{l}\text { overall length of stay } \\
\text { (median / 25-75 percentile) }\end{array}$ & $8(7-10)$ & & $10(8-14)$ & \\
\hline $\begin{array}{l}\text { length of treatment DVT / Pl } \\
\text { (median / } 25-75 \text { percentile) }\end{array}$ & $4(3-6)$ & & $7(6-9)$ & \\
\hline - Intravenous anticoagulantia & $5(3-7)$ & 7 & $7(5-10)$ & 24 \\
\hline - subcut. IMWH and hed rest & $5(4-7)$ & 132 & $5(3-7)$ & 3. \\
\hline - IV anticoagulantia and bed rest & $3(2-4)$ & 9 & $7(5-8)$ & 108 \\
\hline - only subcut. LMWH & & 9 & & 7 \\
\hline Total & & 157 & & 142 \\
\hline
\end{tabular}




\begin{tabular}{|c|c|c|}
\hline & DVT positive & PE positive \\
\hline $\begin{array}{l}\text { Patients meeting DAEP criteria during treatment DVT / PE } \\
\text { (except IV administration of heparin) " }\end{array}$ & $\begin{array}{l}n=44 \\
(28.0 \%)\end{array}$ & $\begin{array}{l}n=73 \\
(51.4 \%)\end{array}$ \\
\hline \multicolumn{3}{|l|}{ Reasons for appropriate stay: $(\%)$} \\
\hline - threatening situations, requiring acute hospital care & 7.8 & 23.2 \\
\hline - care, requiring hospital control or observation & 48.3 & 42.0 \\
\hline - monitoring & 5,8 & 10.1 \\
\hline - infusion and / or medication & 36.6 & 23.2 \\
\hline - nursing care & 0 & 1.4 \\
\hline - other reasons & 1.5 & 0 \\
\hline Overall Inappropriate hospital stay in days (median) & 4 & 3 \\
\hline \multicolumn{3}{|l|}{ Reasons for inappropriate stay: $(\%)$} \\
\hline - delay in diagnostic procedures & 59.3 & 44.4 \\
\hline - application not in time & $(259)$ & $(22.1)$ \\
\hline - not elear defined medical policy & $(14.9)$ & (0) \\
\hline - other reasons & $(18.5)$ & $(22.3)$ \\
\hline - delay in discharge procedures & 29.6 & 38.8 \\
\hline - unavailability of other health care facilities & 7.4 & 5.6 \\
\hline - unavailability of test results & 0 & 11.1 \\
\hline - other & 3.7 & 0 \\
\hline
\end{tabular}

\section{Discussion}

This study showed that the DAEP can be used to assess the appropriateness of hospital stay for DVT and PE patients and can provide reasons why patients need specific hospital care or why patients remain hospitalised inappropriately. The instrument is sensitive to differentiate between different patient groups. The idea of a higher risk for P'E in patients with extensive DVT in not supported by the data found on comorbidity in this and other studies. "If bed rest was indicated, it was mainly due to comorbidity. There was no relation found between the location of DVT and the prescription of bed rest. Except for frequent APT monitoring and specific infusions, there was no indication for hospital stay. So, the appropriateness of hospital stay is mostly determined by reasons not directly related to the treatment of DVT / PE. This study confirms previous research reporting that in most cases hospital stay is inappropriate in treating DVT. '-4 The inappropriate hospital stay in DVT patients is in almost $60 \%$ related to delays in diagnostic procedures. This suggests that there is a substantial delay in the start of the treatment of DVT. This is worrisome as it is good medical practice to start anticoagulant therapy as soon as the diagnosis is suspected. Therefore the use of clearly defined medical policy or clinical pathways and a close monitoring of the process of application for testing can be very useful. Regarding the data in this study, there is reason to believe that there are also possibilities for improving treatment in PE patients. Approximately half of these patients could possibly be treated outside a hospital setting. At this moment, this is already corroborated in a study into this subject. ${ }^{5}$ Yet, further research on this matter is indicated. The retrospective use of an AEP-based instrument depends on accurate record keeping. Due to deficient record keeping data can be missed. 12.11 Although in this study, to our knowledge, only few missing values occurred, this could seriously jeopardise this kind of studies. Therefore, in future research on this matter, using the DAEP 
concurrenti, . . . Lisd. - tages would decrease and more and better data could be collected. Previous studies at the university hospital of Maastricht into the proportion of inappropriate hospital stay ${ }^{9,14}$ showed over $20 \%$ of the total hospital stay to be inappropriate and almost half of this inappropriate stay to be related to delays in hospital procedures (e.g. not clear defined medical policy, delay in diagnostic, therapeutic or discharge procedures). Other international studies ${ }^{15}$ showed an even higher proportion of inappropriate hospital stay $(33-75 \%)$. If inappropriate stay can be reduced, considerable profits in terms of quality, efficiency and costs are possible. Even if medical record keeping (patient files) is not always as perfect as it should be, these kinds of studies can provide useful data to analyse specific patient processes or treatment and the operational rianagement of an organization. The data can also provide additional information leading to agreemerit on medical policy (hospital admission vs. outpatient treatment).

\section{Conciusion}

We demonstrated that the DAEP was sensitive to identify inappropriate hospital stay in the past within a specific patient group for which treatment in an outpatient setting has been proven to be effective. Therefore, it would be plausible to use this tool systematically to assess the current hospital stay of other patient groups in order to reduce or even avoid hospital stay, to increase hospital efficiency and to decrease health care costs. Additional research into other patient groups is still indicated so as to provide guidelines to make the treatment and/or hospital stay as efficient as possible for every patient group. 


\section{References}

1. van der Velde E, Kraaijenhagen R, van Weert H. Turkstra F, Buller H. Survey of the management of deep vein thrombosis in general practice in the Netherlands. Neth J Med. 2000 Oct; 57: 135-41.

2. Schraibman 1, Milne A, Royle E. Home versus in-patient treatment for deep vein thrombosis. (Cochrane Review). In: The Cochrane Library, Issue 4, 2002.

3. Koopman M, Prandoni P, Piovella F, Ockelford P, Brandjes D, van der Meer J, Gallus A, Simonneau G, Chesterman C. Prins M. Treatment of venous thrombosis with intravenous unfractionated heparin administered in the hospital as compared with subcutaneous low-molecular-weight heparin administered at home. The Tasman Study Group. N Engl J Med. 1996 Mar 14; 334: 682-7.

4. van den Belt A, Prins M, Lensing A, Castro A, Clark O, Atallah A, Burihan E Fixed dose subcutaneous low molecular weight heparins versus adjusted dose unfractionated heparin for venous thromboembolism (Cochrane Review). In: The Cochrane Library, Issue 4, 2002. Oxford: Update Software

5. Lim A, Parr D, Stableforth D. Fellows M. Fontaine R, Fegan C. Early discharge and home supervision of patients with pulmonary embolism treated with low-molecular weight heparin. EJIM. 2003; 14: 89-93.

6. Strumwasser I, Paranjpe N, Ronis D, Share D, Sell L. et al. Reliability and validity of utilization review criteria. Appropriateness Evaluation Protocol. Standardized Medreview Instrument and Intensify-Severify-Discharge criteria. Med Care, 1990; 28: 95-111.

7. Act on Quality of Care Facilities (in Dutch). Dutch Law Gazette 341, SDU Publ., the Hague, July 1990

8. Gertman P, Restuccia J. The Appropriateness Evaluation Protocol: A Technique for Assessing Unnecessary Days of Hospital Care. Medical Care 1981; 9: 855-71.

9. Panis L, Pop P, Verheggen F. To stay or not to stay. The assessment of appropriate hospital stay, a Dutch report. Int J Qual Health Care, 2002; 14: 55-67.

10. Lavis J, Anderson G. Appropriateness in health care delivery: definitions, measurement and policy implications. Can Med Assoc 1996: 154:32 1-8

1 1. Partsch H, Blattler W. Compression and walking versus bed rest in the treatment of proximal deep venous thrombosis with low molecular weight heparin. J Vasc Surg $2000 ; 32: 861-9$

12. Ramos-Cuadra A, Marión-Buen J, Garcia-Martin M, et al. Use of the Appropriateness Evaluation Protocol: the role of medical record quality. The Effect of Completeness of Medical Records on the Determination of Appropriateness of Hospital Days. Int j Qual Health Care 1995; 7:267-75.

13. Santos-Eggiman BS, Sidler M, Schopfer D, Blanc T. Comparing results of concurrent and retrospective designs in a hospital utilization review. Int J Qual Health Care 1997; 9:115-20.

14. Panis L, Gooskens M. Verheggen F. Pop P. Prins M. Predictors of inappropriate hospital stay: a clinical case study Int J Quality Health Care 2003; 15: 57-65

15. Lang T, Liberati A. Tampieri A, et al. A European version of the appropriateness Evaluation Protocol. Goals and Presentation. Int J of Technology Assessment in Health Care 1999; 15: 185-97. 

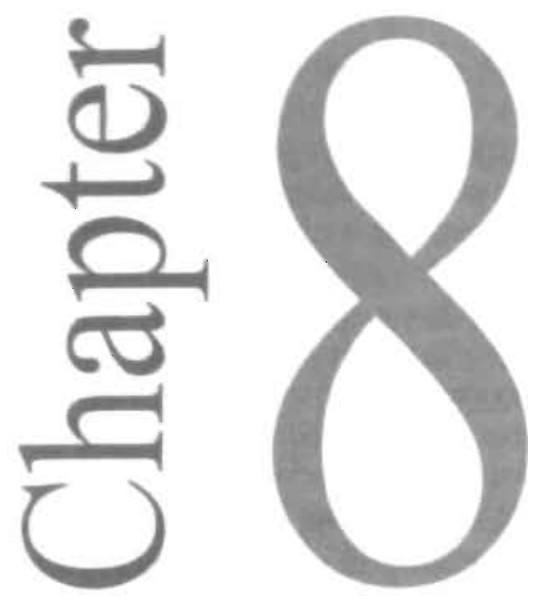

\section{Predictors of inappropriate stay}




\section{ABSTRACT}

Objective:

To assess the reasons for inappropriate hospital stay (IHS) and to identify possible predictors of inappropriate hospital stay.

\section{Design:}

The reasons for IHS were analyzed in a cross-sectional survey at two surgical, one gynecologic and one obstetric ward, using a sample of 610 days of hospital stay by means of the Dutch Appropriateness Evaluation Protocol (DAEP).

\section{Setting:}

The study was conducted at the university hospital of Maastricht, a 715-bed hospital with a regional and teaching function, located in the southern part of the Netherlands.

\section{Results:}

Results indicate that more than $20 \%$ of the hospital stay was inappropriate. The reasons. for IHS were statistically significantly related to the patients' age, the availability of home care and specialism. In a predictive model, only the specialism proved to be a predictor of IHS. Most of the IHS occurred during the first days of hospital stay and the days before the patient's discharge.

\section{Conclusion:}

A substantial proportion of hospital stay was found to be inappropriate, due to hospital procedures and the inability to refer patients to other care facilities or care providers.

Analysis of the causes of IHS provided useful data for improvement actions. Efficient use of hospital resources should be promoted by reducing the delay in interventions and discharge procedures.

Key words: inappropriate hospital stay, IHS, appropriateness evaluation protocol, DAEP. 


\section{Introduction}

Hospital services are the most expensive component of modern health care systems. The total costs of the Dutch health care system over the year 2000 were estimated to be almost 33 billion Euros, being $8.2 \%$ of the Dutch Gross National Product. Intramural care (including hospital services) accounted for almost half of these costs ( 15.4 billion Euros, 14.8 billion US dollar at conversion rate of August $12^{\text {th }} 2002$ ). 'In order to contain these costs, the Dutch government aims to improve the efficiency of the health care services by reducing the costs of these services and by using the capacity of existing health care facilities as optimally as possible. But as a result of various cost-containing measures, waiting lists for inpatient care have risen and are still rising. 'Thus, the remaining hospital beds have to be used as efficiently as possible. One way to achieve this is to avoid inappropriate hospital stay or at least to keep it to a minimum. Reducing this inappropriate stay would improve the productivity of the hospital and reduce the waiting lists. Appropriate stay is considered to be: up to standards, effective, efficient and tailored to the patients' actual needs. ${ }^{2}$ In other words inappropriate hospital stay (IHS) is neither effective (it serves no clinical purpose) nor efficient (resources cannot be used. optimally).

\section{Previous research}

Previous studies ${ }^{3.8}$ at the university hospital of Maastricht assessed ways to measure the inappropriate hospital stay in the Dutch health care setting, resulting in the Dutch Appropriateness Evaluation. Protocol (DAEP). ${ }^{8}$ The DAEP is based on the US Appropriateness Evaluation Protocol (US-AEP). ${ }^{9-12}$ The US-AEP is a widely used tool to assess the appropriateness of the patients' hospital admission or stay. The DAEP consists of 19 diagnosis-independent and care-related criteria to assess the appropriateness of a patient's stay. Our previous studies showed that more than $20 \%$ of the hospital stay was inappropriate and that $45.1 \%$ of this inappropriate stay was due to (internal) hospital procedures, such as delay in discharge or delay in therapy and diagnostics. Specific causes of these delays, however, have not been assessed. Other international studies ${ }^{13-24}$ have also shown that a significant proportion of hospital stay is inappropriate. The DAEP allows us to assess the appropriateness of the patients' stay, but the causes of IHS are not entirely clear.

\section{Objectives}

The objectives of the present follow-up study were:

- To classify the inappropriate stay whether or not it was the result of hospital procedures:

- To assess the average delay in hospital procedures and the causes of these delays;

- To determine possible predictors of these delays;

- To provide possible solutions to these problems.

The present study assessed the appropriateness of the setting, not the appropriateness of care. ${ }^{25}$ Neither the appropriateness. of the patient's admission nor that of professional' services was assessed in this study. 


\section{Methods}

\section{Setting}

This study was conducted at the university hospital of Maastricht. This 715-bed teaching hospital is located in the southeastern part of the Netherlands. In 1999 the university hospital Maastricht admitted 22,000 patients and generated about 200,000 days of stay with an average length of stay of 8.9 days (nationwide 9.2 days). The occupancy rate of the university hospital Maastricht in 1999 was $79.8 \%$.

\section{Assessment of inappropriate stay}

We used the DAEP to assess the appropriateness of stay. The DAEP is a criteria-based decision support tool for determining the medical necessity of hospital admissions and days of care. It consists of sets of generic (diagnosis-independent) and explicit (care related) criteria. The DAEP consists of 19 criteria for appropriate stay, related to medi$\mathrm{cal}$ and nursing services and a list of reasons for IHS. If none of these criteria can be met the day of stay is considered to be "inappropriate" in an acute care hospital and a list of reasons has to be filled out, indicating why the patient is nevertheless staying in the hospital. For our study, appropriate hospital stay was defined as inpatient stay that requires continuous and active medical, nursing or paramedical treatment, which under existing legislation cannot be provided through extramural care, day-care or outpatient care. ${ }^{8}$ The validity and reliability of the DAEP were assessed in previous studies. ${ }^{7.8}$

A flow-chart was designed to facilitate an unambiguous classification of the reasons for IHS into the six main categories (see fig. 1). ${ }^{8}$ Three of these categories, delay in therapy or diagnostics, delay in receiving test results and delay in discharge procedures, can be controlled by the hospital. The hospital management itself however, has no or little control over the other three categories: lack of available alternative care facilities, lack of available primary care and difficulties in the patients' situation at home. Since this study focused only on the 'controllable' inappropriate stay, an Ishikawa diagram was used for an in-depth analysis of these reasons (see fig. 2).

\section{Study sampling and data collection}

For this study two general surgery wards (providing a total of 81 beds for general, vascular and abdominal surgery), one obstetric ward ( 10 beds) and one ward providing 10 gynecologic beds and 14 beds for ophthalmologic short-stay patients (total 24 beds) were selected. During a 6 -month period 610 days of hospital stay were assessed by registered nurses $(n=6)$ of the wards involved. These days of stay were randomly selected by computer program. The stay of each patient, present on the randomly selected days, was reviewed by a concurrent application of the DAEP, assessing the hospital stay during that particular day. Only the selected. day of stay was assessed, not the entire hospitalization period. Data reviewed included the date, the patient registration number, gender, age, the availability of professional or informal home care, medical specialty, ward and the individual scores on the DAEP's criteria or reason list. Also the general data from the hospital information system (date of admission and discharge, length of stay) were used. To facilitate presentation of the data, the patient's age was divided into three categories: less than 30 years, from 30 to 60 years and over 60 years of age. The assessment of the index day was done after the medical and nursing information was exchanged and discussed in the team and the treatment plan was specified. 
Fig. 1: Flowchart to classify inappropriate stan

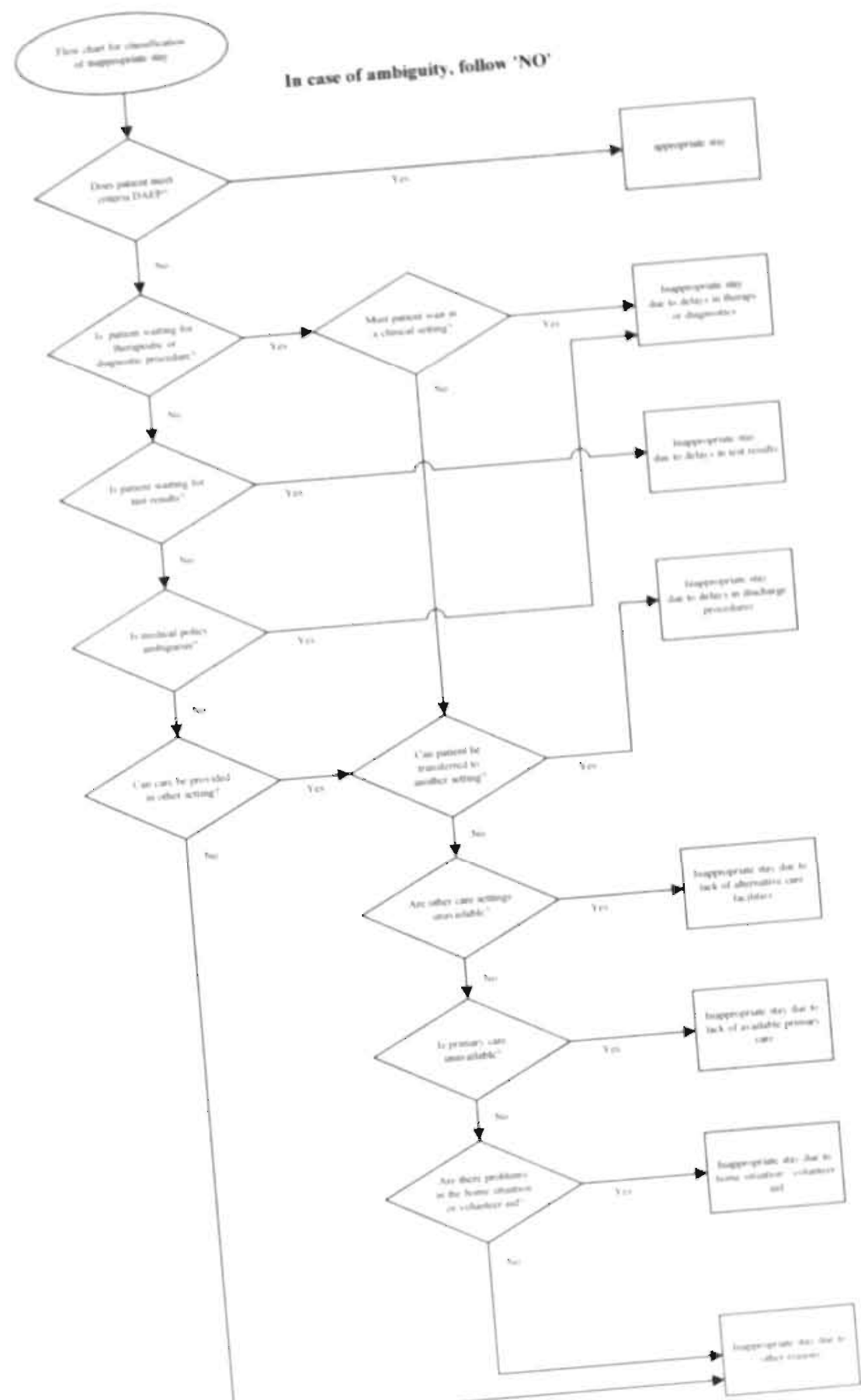


The flow-chart to classify the inappropriate stay was designed with the use of expert opinions from registered nurses on the participating wards. Nineteen versions of this flow-chart were tested before the final version was determined. Agreement in the identification of the reasons for inappropriate stay between nursing staff using the decision tree and nurses not using this decision tree was assessed as a measure for the reliability of the chart. Therefore 49 days of inappropriate stay were assessed by nurses using the flow-chart $(n=3)$ and nurses not using this tool $(n=3)$. To express this agreement, the 'Kappa' was calculated, describing the true agreement as a proportion of the potential agreement (after correction for coincidental agreement). ${ }^{27}$ The reliability of the classification by a flow-chart was high (Kappa: .86).

Fig. 2: Ishikawa diagram on factors leading to delay in therapeufic, diagnosfic or discharge procedures (grouped by face of care)

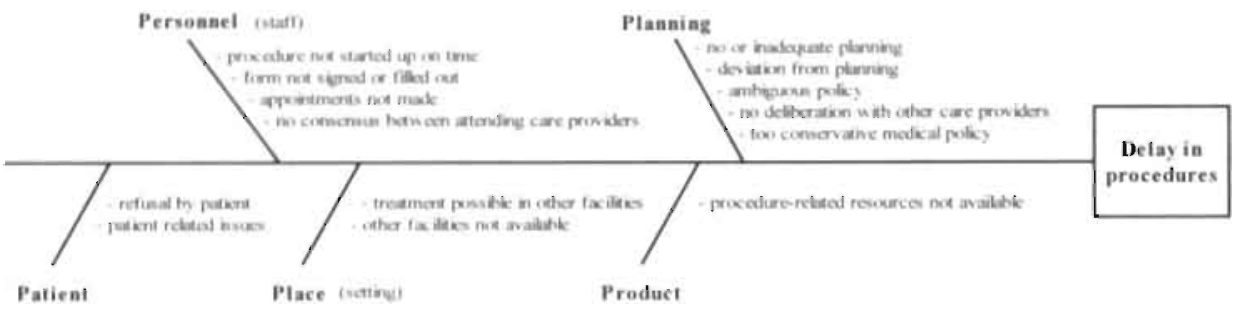

\section{Statistical analysis}

Descriptive analysis was applied to the data concerning the reasons for inappropriate stay. To avoid bias by patient related factors and correlated measurements in the same patient (age, gender, availability of home care, medical specialty, ward) in calculating the significance of the data and predicting their value, all assessed days of stay related to the same patient had to be removed. To avoid bias on gender, the data were also corrected for obstetric and gynecologic patients. We used the Student's t-test to test the mean age between groups with and without IHS. The Pearson Chi-square test was used to test the significance of differences in the rate of appropriateness between groups. The differences in (mean) age between the several types of IHS were analyzed by means of ANOVA. To examine the effect of different factors on IHS, a logistic regression model was estimated. In this model an independent variable found to be statistically significant in a two-way table might turn out to be insignificant when other possible explanatory variables are controlled for. To identify the possible determinants of IHS, all relevant theoretically significant variables were included in the model. The logistic regression model contains the dichotomous variable "appropriate stay" as dependent measure. If the stay was considered appropriate, the variable takes the value of one; otherwise the variable was assigned the value of zero. All data analyses were performed using the SPSS-PC software package, version 10.0. 


\section{Results}

In total we assessed 610 days of stay. After removal of repeated measurements of the same patient, a total of 412 days of hospital stay of individual patients were analyzed further. In this sample, 80 days of stay $(19.4 \%)$ were found to be inappropriate. There was a significant difference in inappropriate stay between the various wards $(p:<.001)$ (see table 1).

Table 1: Inappropriate days of stay by hospital ward

\begin{tabular}{lccc}
\hline Ward & \multicolumn{2}{c}{ Days of inappropriate stay } \\
Surgical ! & 160.8 & 21 & $\begin{array}{c}\text { Iotal days of stay } \\
\text { n }\end{array}$ \\
Surgical 2 & 32.2 & 37 & 125 \\
Obstetrics & 14.8 & 12 & 115 \\
Gynccology / Ophthalmology & 11.0 & 10 & 91 \\
\hline Total & 19.4 & 80 & 412
\end{tabular}

Pearson Chi-square: 17.735, df 3, p: $<001$

Table 2 shows the rates of IHS related to the patient's characteristics. Age, medical specialty and availability of home care were significantly related $(.001<p<.023)$ to the proportion of $\mathrm{HHS}$, while the gender showed no relationship with it $(p, 168)$. The rate of IHS was higher in elderly patients, in vascular and general surgery and in cases of lack of available home care.

Table 3 presents the rates of association of the six main reasons for IIS with a number of patient characteristics. Of these reasons the unavailability of test results $(2.5 \%)$ and lack of available primary care $(1.3 \%)$ were not often found to be related to IHS. Therefore, these two reasons could not be analyzed in detail. Approximately $66 \%$ of the observed IHS was related to two factors: delay in discharge $(27.5 \%)$ and delay in therapeutic or diagnostic procedures $(38.8 \%$ ). Patients waiting for discharge were relatively young (mean age 45.0 ) and mostly female. In over $80 \%$ of these patients home care was available. The delay in discharge occurred especially after abdominal surgery $(22.7 \%)$ and on the obstetric and gynecologic wards $(54.5 \%)$. Limiting the analysis by excluding obstetric and gynecologic patients, still $80 \%$ of these patients were female. However, as said before, gender was not statistically significantly related to the four reasons for IHS. The delay in treatment or diagnostic procedures occurred mainly in general $(29.0 \%)$ and abdominal $(25.8 \%)$ surgery.

A more detailed analysis of the delays in therapeutic or diagnostic procedures is shown in tables 4 and 5 . In defining the potential causes of IHS, the Ishikawa-diagram for the 'controllable' inappropriate stay showed the 'five P's' that are allegedly the sources of IHS: Patient, Personnel (staff), Planning (procedures), Place (setting) and Products (resources) (figure 2). 
Table 2: Rates of inappropriate hospital stay by patient characteristics

\begin{tabular}{|c|c|c|c|c|c|}
\hline & \multirow[t]{2}{*}{$\mathrm{p}$} & \multicolumn{2}{|c|}{$\begin{array}{l}\text { Days of inappropriate } \\
\text { stay }\end{array}$} & \multirow[t]{2}{*}{$\begin{array}{c}\text { Days of appropriate: } \\
\text { stay }\end{array}$} & \multirow{2}{*}{$\begin{array}{c}\text { Total days of } \\
\text { stay } \\
n\end{array}$} \\
\hline & & $\%$ & $n$ & & \\
\hline Age ${ }^{\prime}$ & .023 & & & & \\
\hline$<30$ & & 9.3 & 5 & & 54 \\
\hline $30-60$ & & 17.1 & 31 & & 181 \\
\hline$>60$ & & 24.9 & 44 & & 177 \\
\hline Mean age (years) ${ }^{2}$ & .004 & & & 52.1 & \\
\hline Gender ' & .168 & & & & \\
\hline male & & 23.3 & $3 !$ & & 133 \\
\hline female & & 17.6 & 49 & & 279 \\
\hline Availability of home care & .010 & & & & \\
\hline yes & & 16.3 & 49 & & 300 \\
\hline no & & 27.7 & 31 & & 112 \\
\hline Medical specialty ' & .001 & & & & \\
\hline vascular surgery & & 40.5 & 17 & & 42 \\
\hline general surgery & & 32.7 & 16 & & 49 \\
\hline oncological surgery & & 16.7 & 5 & & 30 \\
\hline limb surgery & & 17.6 & 3 & & 17 \\
\hline abdominal surgery & & 16.7 & 17 & & 102 \\
\hline obstetrics & & 14.8 & 12 & & 81 \\
\hline gynecology & & 10.0 & 8 & & 80 \\
\hline ophthalmology & & 18.2 & 2 & & 11 \\
\hline Total & & 19.4 & 80 & & 412 \\
\hline
\end{tabular}

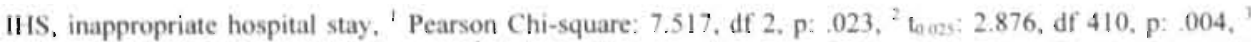
Pearson Chi-square: 1.900, df 1. p: .168, 'Pearson Chi-square: 6.709, df 1. p: 010. OR: 1.960 (95\% Cl: 1.172 - 3.281), 'Pearson Chi-square: 23.704, df 7, p: .001.

Table 4 shows that only four 'P's' were found as reasons for IHS: Patients, Personnel, Planning and Place. The delay in therapy or diagnostics and discharge is especially related to "Planning", while the IHS due to lack of available alternative care facilities is categorized as caused by 'Place'. This table also presents the average delay in days for the different reasons for IHS. If the patient had to wait for treatment or diagnostics, this delay was on average two (2.2) days. As already said, this delay was mainly related to inefficiencies in the application for or planning of these procedures. In $65.3 \%$ of the cases the patient had to wait for an operating room to become available (table 5). This delay occurs in the first days of the patients' stay. A further $27.3 \%$ of this delay was related to diagnostic procedures. The observed IHS occurred at two moments: the first before therapy or diagnostics (mostly right after the admission) and the second before discharge. 
Table 3: Reasons for inappropriafe hosputal stay by patient characteristics

\begin{tabular}{|c|c|c|c|c|c|c|c|}
\hline & $p$ & DT & TR & DP & LF & L.P & DH \\
\hline total IHS & & $38.8 \%$ & $2.5 \%$ & $27.5 \%$ & $21.3 \%$ & $1.3 \%$ & $8.8 \%$ \\
\hline (in days) & & $\left(45.6^{v / 1}\right)$ & $\left(2.9^{v i}\right)$ & $\left(14,7^{n}\right)$ & $\left(250^{17}\right)$ & $\left(1.5^{\prime \prime}\right)$ & $\left(10.3^{17}\right)$ \\
\hline age' & .012 & & & & & & \\
\hline$<0$ & & $<5.0 \%$ & & $18.2 \%$ & . & & \\
\hline $30-60$ & & $51.6 \%$ & & $50.0 \%$ & $5.9 \%$ & & $28.6 \%$ \\
\hline$>60$ & & $45.2 \%$ & & $31.8 \%$ & $94.1 \%$ & & $71.4 \%$ \\
\hline mean age & $<.001$ & 57.1 & 64 & 45.0 & 753 & 78 & 69.6 \\
\hline \multicolumn{8}{|l|}{$(\text { years })^{2}$} \\
\hline gender' & 006 & & & & & & \\
\hline male & $\left(.153^{\circ}\right)$ & $43.7 \%$ & & $\begin{array}{l}9.1 \% \\
(20.07)\end{array}$ & $47.1 \%$ & & $85.7 \%$ \\
\hline female & & $56.3 \%$ & & $\begin{array}{l}90.9 \% \\
(80.0 \%)\end{array}$ & $52.9 \%$ & & $143 \%$ \\
\hline social situation & .008 & & & & & & \\
\hline care available & & $67.7 \%$ & & $81.8 \%$ & $294 \%$ & & $57.1 \%$ \\
\hline no care available & & $32.3 \%$ & & $18.2 \%$ & $70.6 \%$ & & $42.9 \%$ \\
\hline Medical specialty & $<.001$ & & & & & & \\
\hline vascular surgery & & $16.1 \%$ & & $4.5 \%$ & $58.8 \%$ & & $14.3 \%$ \\
\hline general surgery & & $29.0 \%$ & & $4.5 \%$ & $11.8 \%$ & & $57.1 \%$ \\
\hline oncological surg. & & $3.2 \%$ & & - & $17.6 \%$ & & $14.3 \%$ \\
\hline limb surgery & & $3.2 \%$ & & $4.5 \%$ & - & & - \\
\hline abdominal surg. & & $25.8 \%$ & & $22.7 \%$ & $11.8 \%$ & & $14.3 \%$ \\
\hline obstetrics & & - & & $54.5 \%$ & - & & - \\
\hline gynecology & & $19.6 \%$ & & $4.5 \%$ & . & & $=$ \\
\hline ophthaimology & & $3.2 \%$ & & $4.5 \%$ & - & & - \\
\hline
\end{tabular}

IHS: inappropriate hospital stay, DT: Diagnosis \& treatment, TR: Unavailability of test results, DP: Discharge procedures, LF: Lack of available care facilities, LP: Lack, of available primary care, DH: Difliculties at

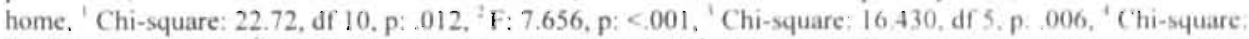
15.576, df 5, p: 008, 'Chi-square: 101.279, df 35, p: <.001. " if obstetrics are excluded; Chi-square 8.054, df 5. p: . 153 . 
Table 4: Relarionship between average delay and reasons for inappropriate hospital stay

\begin{tabular}{|c|c|c|c|c|}
\hline (n) & $\begin{array}{l}\text { Diagnosis and } \\
\text { treatment } \\
\text { (31) }\end{array}$ & $\begin{array}{c}\text { Discharge proce- } \\
\text { dures } \\
\text { (22) }\end{array}$ & $\begin{array}{l}\text { Lack of available } \\
\text { care facilities } \\
\text { (17) }\end{array}$ & $\begin{array}{c}\text { Difficulties at } \\
\text { home } \\
\text { (7) }\end{array}$ \\
\hline Average delay in days & 2.2 & 2.7 & 21.8 & \\
\hline \multicolumn{5}{|l|}{ Due to: } \\
\hline Patient factors & $12.4 \% \quad(4)$ & $18.2 \% \quad(4)$ & $5.9 \% \quad$ (1) & $28.6 \%$ \\
\hline Personnel (staff) factors & $22.6 \% \quad(7)$ & $13.6 \%$ & $5.9 \% \quad(1)$ & - \\
\hline Planning procedures & $61.3 \% \quad(19)$ & $68.2 \%(15)$ & - & $14.3 \%:(1)$ \\
\hline Place (setting) & $3.2 \% \quad$ (1) & - & $88.2 \%(15)$ & $57.1 \% \cdot(4) r$ \\
\hline
\end{tabular}

Table 5: Treatment delays by surgical specialty (\%)

\begin{tabular}{lccc}
\hline Surgical specialty & $\begin{array}{c}\text { Delays due to } O R \\
\text { procedures }\end{array}$ & $\begin{array}{c}\text { Delays due to diagnostic } \\
\text { procedures }\end{array}$ & \begin{tabular}{c} 
Other treatment delays \\
\hline Vascular
\end{tabular} $8^{\text {General }}$ \\
Abdominal & 40.0 & 20.0 & 22.2 \\
\hline Total & 71.4 & 33.3 & 7.4
\end{tabular}

OR: operating room

Predictors for inappropriate hospital stay

Univariately age, availability of home care and medical specialty were statistically significantly related to IHS. However, in the multiple logistic regression model, only specialty proved to be statistically significantly related to IHS, while age, gender and the availability of home care were not. Table 6 shows the eventual logistic regression results with the results for medical specialty controlled for the age and gender of the patient. The chances for IHS in patients in general surgery are 2.87 times higher than those for patients in Obstetrics \& Gynecology (p: .018) and 2.54 times higher than those for patients in abdominal surgery (p: .022). Next to these, the chances for IHS of patients in vascular surgery are 3.50 times higher than for patients in Obstetrics \& Gynecology (p: $.011)$ and 3.10 times higher than for patients in abdominal surgery ( $\mathrm{p}: .006$ ). 
Table 6: Results of logistic regression analysis.

\begin{tabular}{|c|c|c|c|c|c|c|c|c|}
\hline \multirow[t]{2}{*}{ Paticnt characteristics } & \multirow[t]{2}{*}{ B } & \multirow[t]{2}{*}{ S.E. } & \multirow[t]{2}{*}{ Wald } & \multirow[t]{2}{*}{ df } & \multirow[t]{2}{*}{ Sig. } & \multirow[t]{2}{*}{$\operatorname{Exp}(B)$} & \multicolumn{2}{|c|}{$95.0 \%$ C I f for $\operatorname{Exp}$ (B) } \\
\hline & & & & & & & Lower & Upper \\
\hline Gender & 0.104 & 0.304 & 0.117 & 1 & 0.733 & 1.11 & 0.611 & 2.015 \\
\hline Age : & 0.011 & 0.008 & 1.97 & 1 & 0.16 & 1.011 & 0.996 & 1.027 \\
\hline Medical specialty ' & & & 13.098 & 6 & 0.042 & & & \\
\hline Vascular surgery & 1.254 & 0.494 & 6.435 & 1 & 0.011 & 3.503 & 1.33 & 9.227 \\
\hline General surgery & 1.055 & 0.444 & 5.644 & 1 & 0.018 & 2.871 & 1.203 & 6.854 \\
\hline Oncological surgery & 0.241 & 0.562 & 0.184 & 1 & 0.668 & 1.273 & 0.423 & 3.832 \\
\hline Ophthalmology & 0.308 & 0.85 & 0.131 & 1 & 0.717 & 1.361 & 0.257 & 7.198 \\
\hline Limb surgen & 0.25 & 0.716 & 0.122 & 1 & 0.727 & 1.284 & 0.315 & 5.225 \\
\hline Abdominal surgery & 0.123 & 0.432 & $0.08 i$ & 1 & 0.776 & 1.131 & 0.485 & 2.637 \\
\hline Constant & -2.506 & 0.506 & 24.532 & 1 & 0 & 0.082 & & \\
\hline
\end{tabular}

Dependent variable is type of stay $(0=$ appropriate; 1 - inappropriate). 'Using backward elimination. the change in -2 $\log$ likelihood Chi-square for specialty was: 12.85, df 6, p: 045 and for the availability of home care: 1.752, df 1. p: 186, Because age and specialty turned out to be related to each other (one-way ANOVA, F: 33.982 by 6 and 404 df $(\rho:-001)$ the odds ratio for age was not significant anymore. 'In this table Obstetrics \& Giynecology are compared with the other specialties.

\section{Discussion}

Although the overall mean length of stay in the university hospital of Maastricht (8.9 days) is lower than the national mean length of stay (9.2 days), ' the IHS rate in this study is about $20 \%$, which is consistent with the findings in our previous studies. in This study shows that it is possible to identify predictors for IHS. However, it is still unclear how to proceed. How can we actually reduce IHS? In the search for an efficient care system IHS must be avoided. However, the aim of 'zero IHS' is a utopian dream. There will always be factors uncontrollable by the organization, e.g. the lack of available alternative care facilities. According to the data in table 3, approximately $65 \%$ of the IHS ( $38.8 \%$ and $27.5 \%$ ) could be avoided if corrective measures were made. This amounts to $12.9 \%$ of the hospital stay in this study. By reducing the IHS more patients could be treated and waiting lists would be decreased, although the workload of the hospital staff and specific hospital costs would probably increase.

A substantial part of the IHS is related to delays in diagnostics and delays in operation room procedures. Since most surgical admissions are planned in advance, some of the corresponding diagnostic procedures could be carried out on an outpatient base. Subsequently, the operation could be performed on the day of admission and a great amount of this type of IHS would be avoided. The current situation may be related to the fact that professionals often try to control the environment in which the treatment -including the diagnostics- takes place. During the patients' stay, IHS usually occurs during two phases of the treatment. These are the transfer of the patient from one department to an other, such as from the ward to the operating room and vice versa or from the hospital to the home situation (discharge). There are several possibilities for reducing this type of IHS in clinical practice. To avoid waiting for diagnostics, the criteria for the use of 
certain diagnostics could be tightened and the subsequent compliance increased. This would mean less but more rational use of these facilities. At this moment non-acute diagnostics are not performed outside ordinary business hours. If diagnostics were performed outside business hours capacity would increase by about $60 \%$, since at this moment the existing capacity is only in use in one third (i.e. 8 hours) of a day. To do so would revolutionize the Dutch health care system. But this is still an emotive subject. Alternatively, additional equipment could be installed, although both these options would also mean an increase in costs.

The causes of the delay in treatment have not been specified in this study. Possible causes for this type of IHS might be: insufficient staff or poor operation room (OR) scheduling. If so, this type of IHS could be decreased by appointing more staff or adjustment of the OR scheduling. Certainly the first option would again mean an increase in costs. This argues for a better overall planning of processes and the use of protocols or clinical pathways ${ }^{26}$ for improving the flow of different patient groups. This planning would link the several phases of patient care more efficiently.

Another part of IHS occurs before discharge. The literature shows that many hospitals are struggling with this issue. ${ }^{27.32}$ If discharge procedures were dealt with at the moment that the patient has no further need of hospital stay, the patient could be discharged and be treated elsewhere even better. To start planning the discharge procedure even before the patient's admission could be a useful concept for change, while 'minimalcare facilities' might be appropriate to provide the needed care.

Patient characteristics might be useful in predicting IHS. As the age of the patient increases, the IHS rates show an upward trend. The availability of home care affects IHS rates in a positive way. And the IHS is related to the medical specialty. If a patient. matches one or more of these characteristics ( $>60$ years of age, lack of available home care, vascular or general surgery) extra attention should be paid in avoiding IHS. This attention should focus on the planning of and the compliance with therapeutic, diagnostic or discharge procedures. Furthermore, the availability of other care facilities should. be improved, although this is hard for the hospital to control by itself. The 'minimalcare ward' mentioned before where patients can recover outside a hospital setting could help to address this problem.

\section{Future research}

Several practical suggestions to reduce IHS, have been mentioned above. Implementing: these suggestions without other efforts to address the waste of quality care will certainly increase hospital costs. Thus, cost-benefit analyses should be made in order to select the best solution(s). Although this study gives some insight into causes and predictors of IHS, several questions remain to be answered. How should we proceed in order to reduce IHS in clinical practice? What are the effects of reducing IHS on the workload of hospital staff? Might the reduction of IHS in one part of the organization result in an increase of IHS in another? Why is there a significant difference in IHS between several wards? Can this difference be explained by the type of patient, e.g. obstetric patients who are relatively young and for whom home care is available? These questions have to be answered in future research.

In this study we focused on those factors for IHS that can be controlled by the hospital. But as seen in this study, patients designated for transfer to other care facilities wait on average more than 20 days for their transfer. Much profit could be gained by reducing this type of $1 \mathrm{HS}(5 \%$ of all days of stay, average length 22 days) and this also requires further research. 


\section{Conclusions}

The rate of IHS measured in this study $(19.4 \%)$ was consistent with our previous studies. IHS was caused mainly by delays in diagnosis or treatment $(38.8 \%)$, delays in discharge procedures $(27.5 \%)$ and lack of available alternative care facilities $(21.3 \%)$. The age of the patient, the availability of home care and the medical specialty were statistically proven to be predictors for IHS. Elderly patients (over 60 years) show higher rates of IHS. There was a significant difference of IHS between the several medical specialties. Finally, the IHS occurs mostly during the first days after admission and the days before discharge.

\section{Acknowledgements}

We would like to thank F. H. M. Nieman, Ph.D, for checking the statistical analysis and in particular the logistic regression model. 


\section{References:}

1. Statistics Netherlands, Annual report (in Dutch), the Netherlands, 2000.

2. Act on Quality of Care Facilities (in Dutch). Dutch Law Gazette 341. SDU Publ., the Hague, July 1996.

3. Schut M. Appropriate use of hospital beds. A study of the possibilities of the AEP (in Dutch). Maastricht University, Maastricht, 1994.

4. Esveld S. The Appropriateness Evaluation Protocol. A study of the reliability and validity of the AEP (in Dutch). Maastricht University, Maastricht, 1995.

5. Smeets P, Pop P, Verheggen F. Assessing the appropriateness of hospital stay: the possibilities of the appropriateness evaluation protocol (in Dutch). Med Contact. 1997; 52: 757-60.

6. Smeets P, Pop P, Verheggen F. Assessing the appropriateness of hospital stay: features of quality assurance and utilization review tools (in Dutch). Med Contact 1997; 52: 760-61.

7. Smeets P, Verheggen F, Pop P, Panis L. Carpay J. Assessing the necessity of hospital stay by means of the appropriateness evaluation protocol: how strong is the evidence to proceed? Int J Qual Health Care, 2000; 12: 483-93.

8. Panis L. Pop P, Verheggen F. To stay or not to stay. The assessment of appropriate hospital stay, a Dutch report. Int J Qual Health Care, 2002; 14: 55-67.

9. Gertman P, Restuccia J. The Appropriateness Evaluation Protocol: A Technique for Assessing Unnecessary Days of Hospital Care. Med Care 1981; 9: 855-71.

10. Restuccia J. The Effect of Concurrent Feedback in Reducing Inappropriate Hospital Urilization. Med Care 1982; 20:46-62.

11. Restuccia J. Appropriateness Evaluation Reviewers' Manual. In: The AEP Final Report. Springfield, VA: National Technical Information Service, 1986 Publication No. PB87-112041.

12. Restuccia J. The evolution of Hospital Unilization Review Methods in the United States. Int J Qual Health Care 1995; 7: 253-60.

13. Baré M. Prat A. Lledo L. Asenjo M. Salleras L. Appropriateness of admissions and hospitalization days in an acute-care teaching hospital. Rev Epidém et Santé Publ 1995: 43:328-36.

14. Bentes M, Gonsalves M, Santos M, Pina E. Design and development of a Utilization Review Program in Portugal. Int J Qual Health Care 1995; 7: 201-12.

15. Fellin G. Apolone G, Tampieri A, et al. Appropriateness of Hospital use: an Overview of Italian Studies. Int J Qual Health Care 1995: 7: 219-25.

16. Kaya S. Erdem Y. Dogrusoz S. Halici N. Reliability of a hospital utilization review method in Turkey: Int J Qual Health care 1998; 10: 53-8.

17. Lang T, Liberati A. Tampieri A, et al. A European version of the appropriateness Evaluation Protocol. Goals and Presentation. Int J of Technology Assessment in Health Care 1999; 15: 185-97.

18. Lorenzo S, Sunol R. An overview of Spanish Studies on Appropriateness of Hospital Use: Int J Qual Health Care 1995; 7: 213-8.

19. Lorenzo S, Beech R, Lang T, Santos-Eggiman B. An experience of utilization review in Europe: sequel to a BIOMED project. Int J Qual Health Care 1999; 11: 13-9.

20. Robain M, Lang. T. Fontaine A, et al. Reliability and validity of the French version of the first part of the Appropriateness Evaluation Protocol (.AEPf): criteria of appropriateness of hospital dans (in French). Rev Exidém et Santé Publ 1999: 47: 139 49. 
21. Santos-Eggiman B, Paccaud F. Blanc T. Medical Appropriateness of Hospital Utilization: an Overview of the Swiss Experience. Hospital utilization reviews under field conditions: Potential and Improvements. Int. J Qual Health Care 1995; 7: 227-32.

22. Merom D, Shohat T, Harari G, Oren M, Green M. Factors associated with inappropriate hospitalization days in internal medicine wards in Israel: a cross-national survey. Int J Qual Health Care 1998; 10: 155-62.

23. Paldi Y, Porath A, Friedman L. Mozes B. Factors Associated with Inappropriate Hospitalization in Medical Wards: a Cross-sectional Study in Two University Hospitals. Int J Qual Health Care 1995; 7: 261-5.

24. Black N. Appropriateness of medical care in Europe; a commentary. Int J Qual Health Care, 1994; 6: 231-2.

25. Lavis J, Anderson G. Appropriateness in health care delivery: definitions, measurement and policy implications. Can Med Assoc J 1996; 154:321-8

26. Bouter L, van Dongen M. Epidemiologic research, design and interpretation. Bohn, Stafleu, Van Loghum. Houten/Diegem 1995.

27. Gregor C, Pope S, Werry D. Dodek P. Reduced length of stay and improved appropriateness of care with a clinical path for total knee or hip arthroplasty: I Qual Improvement 1996; 22: 617-28.

28. Dautzenberg $\mathrm{P}$, Bremer R, Lormans $\mathrm{M}$. Tanis J. Delay in discharge in general hospitals (in Dutch). Med Contact 1997; 52: 1310-13.

29. McWilliam C, Sangster J. Managing patient discharge to home: the challenges of achieving quality of care. Int J Qual Health Care 1994; 6: 147-61.

30. Moons P, Steeman E, Wouters B et al. Discharge management in Belgian general hospitals (in Dutch). Acta hospitalia 1999; 4: 45-56.

31. Pavlin, D, Rapp, S. Factors affecting discharge time in adult outpatients. Anesth. analg 1998; 87:816-26.

32. Marshall SI, Chung F. Discharge criteria and complications after ambulatory surgery. Anesth analg 1999; 88: 508-17. 


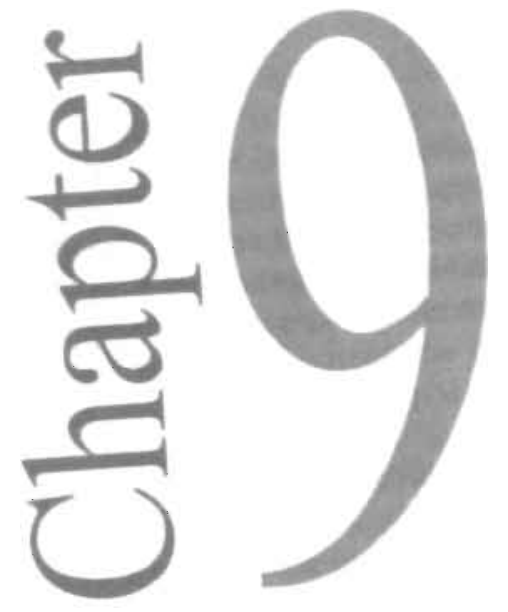

Flaws in patient flows

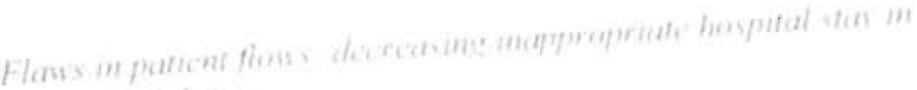

Submitted as: Intermal Medictm

Panis I. Verhegeen 1. Pop P. Prims M

Qual saf Health Care 


\section{Abstract}

\section{Background.}

Hospital beds have been reduced during the past decades. However, the population is ageing and the demand for hospital admissions increases. This requires an optimal use of the remaining hospital beds, providing the right care in the right setting. Therefore hospital stay should as short and as appropriate as possible. Previous studies at the university hospital of Maastricht showed a large proportion of the stay to be inappropriate, often related to delays in specific hospital procedures.

\section{Objectives.}

To decrease inappropriate hospital stay related to delays in diagnostic procedures and to hospital discharge within the department of internal medicine.

\section{Methods.}

The Dutch Appropriateness Evaluation Protocol (DAEP) was used to assess the appropriateness of hospital stay. Additionally, control charis ( $\mathrm{P}$-charts) were used to monitor the processes of hospital discharge and diagnostics and to differentiate between normal and abnormal variance. To reduce the delay is diagnostic procedures 'slots' (i.e. reserved time for a specific diagnostic procedures) were set up. To reduce the delay in hospital discharge an "admission \& discharge team" was introduced.

\section{Results}

Only the actions to improve the process of hospital discharge could be proven to be successful in a statistically significant way. However, the inappropriate hospital stay related to delays in diagnostic procedures did not differ. P-charts showed the variation. of this process to remain within the control limits. The inappropriate hospital stay related to the unavailability of other health care facilities increased by $6.7 \%$.

\section{Conclusions}

The improvements in the procedures of hospital discharge, in order to reduce inappropriate hospital stay, were proven to be successful. However, the impact of these improvement actions was undone by the increase of inappropriate hospital stay caused by the unavailability of other health care facilities.

Keywords: Inappropriate hospital stay, Internal Medicine, delay, hospital discharge. diagnostic procedures 


\section{Introduction}

As a result from cost containment actions the number of hospital beds has been reduced during the past decades. However, the population is ageing and the demand for hospital admissions increases. This requires an optimal use of the remaining hospital beds, providing the right care in the right setting. During hospital stay, patients go through diagnostic and / or therapeutic procedures requiring specific medical and nursing care. But hospital stay is also accompanied by an increased risk of complications, such as infections, which affects the medical outcome and increases health care costs. This is another reason to aim for a hospital stay that is as short and as appropriate as possible. Appropriate hospital stay (for our study defined as: "Hospital stay requiring continuous and active care, which under existing legislation, cannot be provided through extramural care, daycare or outpatient care) ' has been extensively widely been studied in the United States over the last 20 years and in Europe over the last decade. ${ }^{2-7}$ The rate of inappropriate hospital days has been defined as the ratio between the cumulative number of inappropriate days over the total number of days of hospital stay. This rate varies from 18 to $50 \%$ in reports from the United States and from 10, to $74 \%$ in Europe. Previous studies at the university hospital of Maastricht on the appropriateness of hospital stay showed that a large proportion of this stay (on average $20.6 \%$ ) was inappropriate. ${ }^{1.8}$ Follow up studies into the reasons of this inappropriate hospital stay (IHS) revealed specific reasons for this IHS. One half of this IHS was related to 'external factors': patients could not be transferred to other health care facilities or be discharged from the hospital because of the lack of certain health care facilities. The other half of the IHS was related to 'internal factors' caused by delays in specific hospital procedures such as diagnostics and therapy or the patients' discharge from the hospital. The reduction of IHS may be achieved by the improvement of patient management or patient logistics. The objective of this study was to decrease this latter IHS within the medical department of internal medicine. Therefore specific improvement actions were set up. In this paper we examined the overall results of these interventions on the IHS and the specific effect of these actions separately. 


\section{Methods}

\section{Setting}

This study was conducted at the department of Internal Medicine of the university hospital of Maastricht (azM), a 715-bed hospital with a teaching and a district function, located in the southeastern part of the Netherlands. In 2002. the university hospital Maastricht admitted 22,000 patients and generated about 200,000 days of stay with an average length of stay of 8.9 days (nationwide 9.2 days). (Internal Medicine: 2777 admissions, 29983 days of stay, length of stay 10.8 days)

\section{The assessment of IHS}

We used the Dutch Appropriateness Evaluation Protocol (DAEP) to assess the appropriateness of hospital stay. The DAEP is a criteria-based decision support tool to assess the appropriateness of hospital stay. It consists of 19 criteria for appropriate stay, (diagnosis-independent and care related) and a list of reasons for inappropriate hospital stay (IHS). A day of stay is considered to be "inappropriate" if none of the criteria can be met. In that case, a list of reasons has to be filled out, indicating why the patient is still staying in the hospital. The validity and reliability of the DAEP were assessed in previous studies. ${ }^{1.9}$ To monitor the processes of hospital discharge and diagnostics, control charts (P-charts) were used. ${ }^{10}$ With these control charts process information can be gathered and analysed. Control charts enable to differentiate between normal and abnormal variance.

\section{Interventions to reduce inappropriate hospital stay}

Although data in our previous studies showed that the IHS in the university hospital in Maastricht was relatively low, in comparison to results of other international studies, still almost one quarter of the hospital stay was labelled as 'inappropriate'. Based on the data of our previous studies ${ }^{1.9}$ factors related to IHS were rated on their overall impact and their potential for improvement (fig 1). Two factors scored high on both criteria: delay in hospital discharge, delay in diagnostic procedures. Also the IHS within the department of Internal Medicine was relatively high (21\%). Similar results were found in literature. ${ }^{11-20}$ Therefore the management of that department decided to endorse actions aimed at reduction of IHS.

To reduce the delay is diagnostic procedures 'slots' (i.e. reserved time for a specific department within the regular program) were set up. This implied daily reserved time for two computer tomographies (CT), two ultrasonic procedures within the department of Radiology and one endoscopic procedure within the department of Gastroenterology.

To reduce the delay in hospital discharge an 'admission \& discharge team' was introduced. The purpose of this team was to closely monitor the appropriateness of the patients" admission and to determine the medical policy (including the planned length of hospital stay (LOS)) within 48 hours in case of an emergency admission. Subsequently, this team assessed daily the appropriateness of the patients further hospital stay and saw to the planning and the implementation of the hospital discharge as soon as possible. 


\section{Study sampling and data collection}

A longitudinal cohort study was conducted within the department of Internal Medicine (88 beds). During an 18-month period (November 2001- May 2003) almost 3,500 days of stay were assessed, before and after the implementation of interventions to reduce IHS. During the periods of monitoring, the stay of each patient was reviewed by a concurrent application of the DAEP, assessing the appropriateness of the hospital stay that particular day. Only the stay during the selected periods was assessed, not the entire hospitalization period. Data reviewed included the date, the patient registration number, gender, age, the availability of professional or informal home care, medical specialty, ward and the individual scores on the DAEP criteria or reason list. Also the general data from the hospital information system (date of admission and discharge, length of stay) were used. The assessment of the index day was done after the medical and nursing information was exchanged and discussed in the team and the treatment plan was specified. The DAEP software package, linked to the hospital information system was used by the deputy head nurses $(n=6)$ of the different wards $(n=3)$ to assess the appropriateness of hospital stay.

\section{Statistical analysis}

Descriptive analysis was applied to the data concerning the reasons for inappropriate stay. The data were stratified to avoid bias on gender. We used the Student's t-test to test the mean age between groups before and after implementation. The Pearson Chisquare test was used to test the significance of differences in the rate of appropriateness between groups. To analyse variation in occurrence of IHS control charts ( $P$-charts) were used. All data analyses were performed using the SPSS-PC software package, version 10.0 .

Fig I: Selection of improvement actions impact on IHS vs potential for improvement

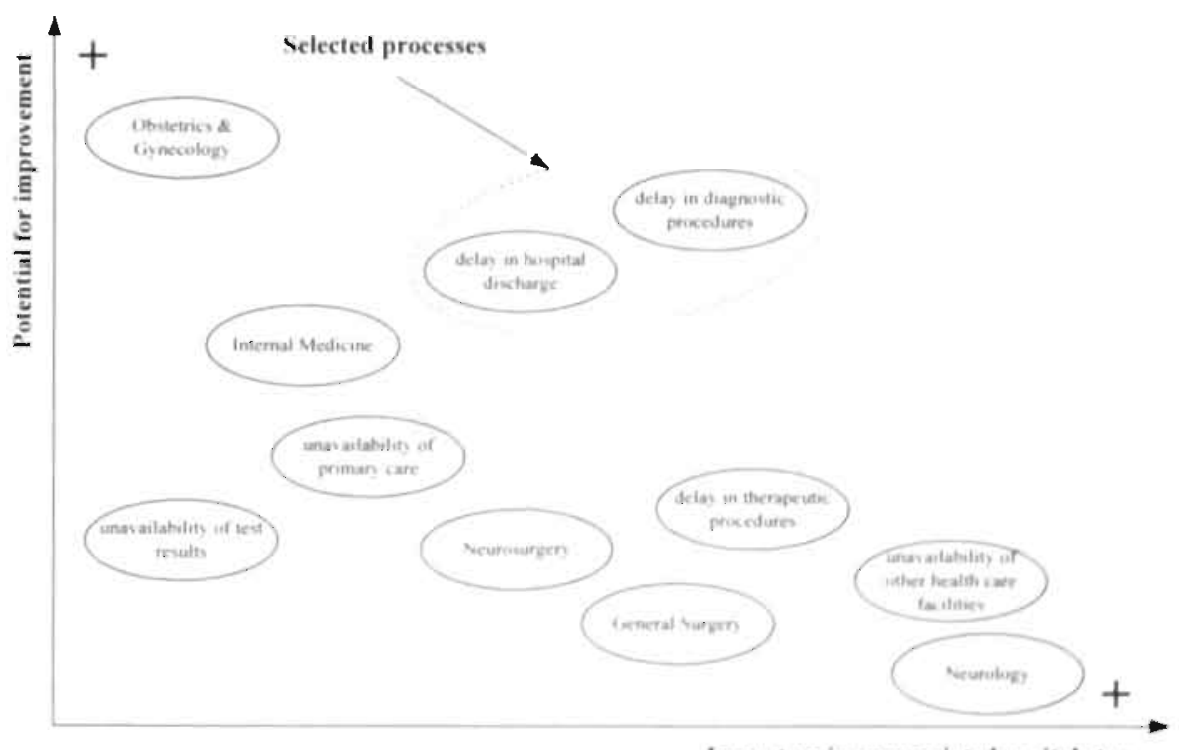

Impact on inappropriate hospital stay 


\section{Results}

In total 3359 days of hospital stay were assessed in 366 patients. Some demographic and clinical characteristics are given in table 1. Although there was a shift in the male / female ratio, the cohorts of 2001 and 2003 did not differ in a statistically significantly way. Overall, 799 days (23.8\%) were labelled as 'inappropriate stay'. Any IHS occurred in 171 of the 366 patients $(46.7 \%)$. During the study, the total IHS within the department of Internal Medicine increased by $4.4 \%$, from 21.2 to $25.6 \%$ (p: 0.003 ).

Table 1: Sample size characteristics

\begin{tabular}{|c|c|c|c|}
\hline & 2001 & 2003 & Total \\
\hline Patients & 154 & 212 & 366 \\
\hline Gender $M / F$ & $69 / 85$ & $115 / 97$ & $184 / 182$ \\
\hline Mean age (SD) & $55.9(17.7)$ & $56.9(19.1)$ & $56.5(18.9)$ \\
\hline $\begin{array}{l}\text { Type of admission } \\
\text { (emergency/elective) }\end{array}$ & $74 / 80$ & $119 / 93$ & $193 / 173$ \\
\hline \multicolumn{4}{|l|}{ Days of stay $(\%)$} \\
\hline Endocrinology. & $65(4.8)$ & $214(10.7)$ & $279(8.3)$ \\
\hline Gastroenterology & $97(7.1)$ & $405(20.3)$ & $502(1+9)$ \\
\hline Geriatry & $169(12.4)$ & $131(0.6)$ & $300(8.9)$ \\
\hline Hematology / Oncology & $300(22.0)$ & $489(24.5)$ & 789 (23.5) \\
\hline Immunology & $20(1.5)$ & $64(3,2)$ & $84(2.5)$ \\
\hline Nefrology & $149(10.9)$ & $198(9.9)$ & $347(10.3)$ \\
\hline Reumatology & \|\|$(8 \|$ & $64(3.2)$ & $(75.5 .2)$ \\
\hline General Int Medicine & $453(33.2)$ & $430(21.5)$ & $883(26.3)$ \\
\hline Total & 1364 & 1995 & 3359 \\
\hline
\end{tabular}

Delan's due to diagnosfics and hospiral discharge

Overall, the IIIS related to delays in diagnostics accounted for $8.2 \%$ (276/3359 days). The difference in frequency of IHS due to delays in diagnostics did not differ between 2001 and $2003(7.5$ vs. $8.7 \%$, respectively, p: 0.246$)$. The overall IHS related to delays in hospital discharge was $4.6 \%$ (153/3359 days). The difference in frequency of IHS due to delays in hospital discharge did differ statistically significantly between 2001 and 2003 (6.6 vs. $3.8 \%$, respectively, p: $<0.001$ ) (table 2). Corrected for gender, these results did not change. 
Table 2: Results on inappropriate hospital stay

\begin{tabular}{|c|c|c|c|c|c|}
\hline \multirow[t]{2}{*}{ Year } & \multirow{2}{*}{$\begin{array}{c}\text { Days of } \\
\text { appropriate hospital stay } \\
(\%)\end{array}$} & \multicolumn{3}{|c|}{$\begin{array}{l}\text { Days of } \\
\text { inappropriate hospital stay }\end{array}$} & \multirow[t]{2}{*}{ Total } \\
\hline & & $\begin{array}{c}\text { Delay in diagnostic } \\
\text { procedures }\end{array}$ & $\begin{array}{c}\text { Delay in hospital } \\
\text { discharge }\end{array}$ & Other delans & \\
\hline 2001 & $1075(78.8)$ & $103(7.5)$ & $90(6.6)$ & $96(7.0)$ & 1364 \\
\hline 2003 & $1485(74.4)^{3}$ & $173(8.7)^{2}$ & $63(3.2)^{3}$ & $274(13.7)^{6}$ & 1995 \\
\hline Total & 2560 & 276 & 153 & 370 & 3359 \\
\hline
\end{tabular}

'Pearson Chi-square: 8.558 , df I. p: 0.003

'Pearson Chi-square: 1.348, df 1, p: 0.246

'Pearson Chi-square: 22.055, df 1. p: $<0.001$

'Pearson Chi-square: 37.059 , df 1. p: $<0.001$

Figure $2 \& 3$ depict the total hospital stay, divided into appropriate stay and the types of IHS in relation to the different units of Internal Medicine. The IHS related to delays in hospital discharge decreased in all departments, except for general Internal Medicine, where it remained the same. In particular, Geriatrics and Reumatology showed the highest decrease. The IHS related to delays in diagnostic procedures increased to a large extend in almost all departments, especially within general Internal Medicine. In the department of Haematology-Oncology almost no IHS occurred. This can be related to the use of strict protocols and planning in the patient's therapy (e.g. cytostatics).

Fig. 2: Total hospital stan 2001

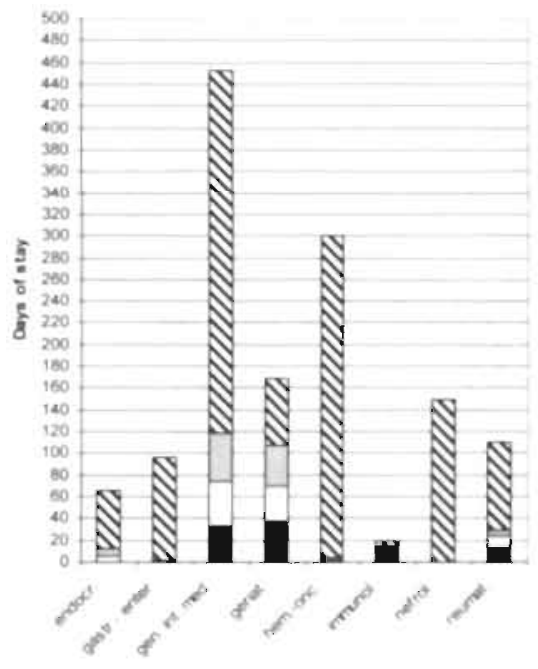

Fig. 3: Total hospital stan 2003

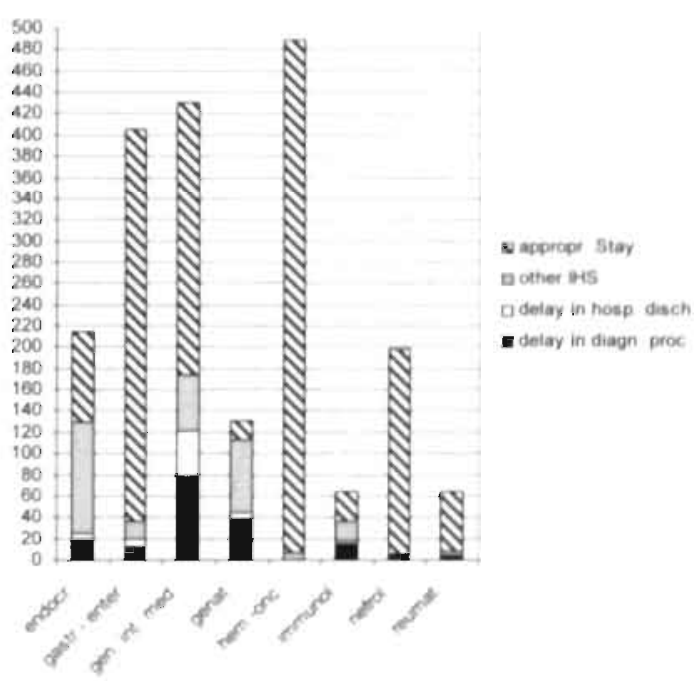


The non-significant decrease in IHS related to discharge procedures (table 2) is depicted in and confirmed in figure 4 . This figure shows this IHS as a proportion of the total hospital stay. There is no sign of any stabilization of the process. On the other hand, however, the process of hospital discharge did show stabilization and a significant decrease in this type of IHS (fig 5). This indicates that the 'admission \& discharge team' operates in an effective way.

Fig. 4: P-chart: Delay in diagnostic procedures

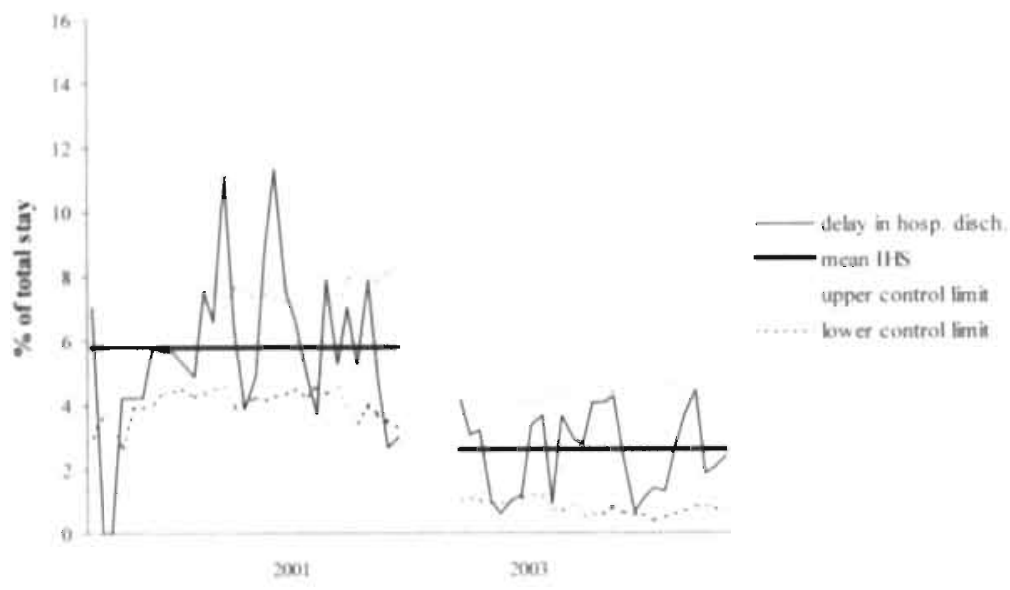

Fig. 5: P-chart Delas in hospifal discharge

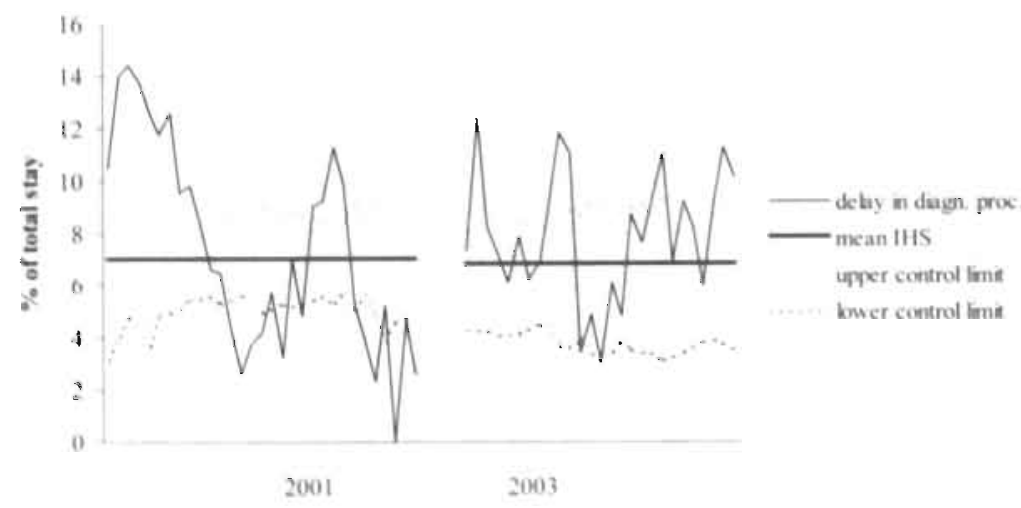




\section{Other causes of IHS}

The increase in total IHS was related to the 'external factors'; mostly these patients were staying in the hospital without medical indication and with, as yet, no options for a transfer to other care facilities or to the home situation. The proportion of this type of IHS almost doubled (7.0 to $13.7 \%$ ) and increased in every department, especially in Endocrinology, Geriatrics and Immunology (fig. $2 \& 3$ ). Table 3 shows that during this study there was an evolution in the relation between the types of hospital admission (elective vs. emergency) and the occurrence of IHS.

In relation to the year 2001 more emergency admissions occurred in 2003 (52\% vs. $57 \%$ ) and the proportion of admissions leading to IHS increased in that same year from $42 \%$ to $50 \%$. In 2003 the relation between type of admission and the occurrence of IHS could be proven statistically significant (p: $<0.001)$. During this study, the 'long' IHS ( $>6$ days) increased. This increase was especially apparent among emergency admissions, where it rose from $3.9 \%(6 / 81)$ to $20.5 \%(25 / 122)$.

Table 3: Type of admission vs. inappropriate hospital stay

\begin{tabular}{lccccc}
\hline & Appropriate hospital stay & \multicolumn{2}{c}{ Inappropriate hospital stay } & Total \\
& & $1-3$ days & $4-6$ days & $>6$ days & \\
\hline Elective admission & 48 & 12 & 5 & 8 & 73 \\
Emergency admission & 41 & 25 & 9 & 6 & 81 \\
\hline Total & 89 & 37 & 14 & 14 & 154
\end{tabular}

Pearson Chi-square: 6.148, df: 3.p: 0.105

\begin{tabular}{lccccc}
\hline & Appropriate hospital stay & \multicolumn{2}{c}{ Inappropriate hospital stay } & Total \\
& & $1-3$ days & $4-6$ days & $>6$ days & \\
\hline Elective admission & 65 & 16 & 4 & 5 & 90 \\
Emergency admission & 41 & 45 & 11 & 25 & 122 \\
\hline Total & 106 & 61 & 15 & 30 & 212
\end{tabular}

Pearson Chi-square: 31.713 , df: 3. p $<0.001$

\section{Discussion}

This study shows the results on our attempt to improve the efficiency of two specific hospital processes. These improvement actions were based on causes of inappropriate hospital stay as they were identified in previous research. These actions focused on reducing IHS related to delays in hospital discharge and to delays in diagnostic procedures. This study was set up as a full quality improvement cycle that identified a problem, attempted to correct the processes responsible for it, and evaluated the results of improvement actions within the internal medicine wards in the university hospital of Maastricht. Although the proportion of overall IHS increased, both the proportions of IHS on 'internal factors' (i.e. delays in hospital discharge and in diagnostic procedures) decreased. However, only the actions to improve the process of hospital discharge could be proven to be successful. Most likely, this success is related to the fact that there was 
a continuous monitoring, evaluation and feed back in this process, while this was less in the process of applying for diagnostic procedures.

There was a remarkable increase in IHS related to 'external factors' (unavailability of other health care facilities an primary care). Although it could not be proven statistically, it is plausible that the 'long' IHS is related to the unavailability of other health care facilities outside the hospital. These 'external factors' are responsible for the increase in overall IHS during this study. A hospital has little control over these factors. 'Blocked beds' ${ }^{21.22}$ (i.e. patients staying in the hospital without medical indication and with, as yet, no options for a transfer to other care facilities) are an example of these factors. So, in future extra attention has to be paid in dealing with this specific type of IHS. The set-up of a 'low-care ward' or a 'care-hotel', age related geriatric services or extended home care ${ }^{22}$ might be options. These services can help the patient to recover outside a hospital setting and result in a more appropriate use of hospital resources. Some inappropriate days of stay due to internal reasons (e.g. delay in hospital discharge) might also decrease, by the use of these facilities. The issue that should be considered is: "What is more cost-effective; extra capacity of extramural health care facilities or IHS within an acute care hospital?".

Even if specific improvement actions on reducing IHS are successful, the entire problem of IHS should be dealt with in order to decrease the total IHS. Otherwise, if IHS continues to exist, the profit in reducing 'internal IHS' remains flooded by the problems of 'external IIIS'. However, this does not release hospitals from their obligation to optimalize their processes. Although these figures on IHS are relatively low, in relation to other international studies, they remain significant. ${ }^{2-4}$

Within the design of this study there are some limitations. We cannot rule out, for example, a prior trend to the reduction of in inappropriate admission and stays, or the possible existence of other concomitant changes that might have affected the results. The estimates of inappropriateness could be conservative, as a day of stay could be rendered 'appropriate' by performing an unnecessary intervention, such as placing an intravenous line in a patient who did not need it. Questioning the appropriateness of each medical procedure might have resulted in even higher estimates of inappropriate care. However, since the selected processes were under full control of the Department of Internal Medicine and since the implementation of the improvement actions was implemented only in that department, we think that these potential biases did not substantially influence the resuits.

Oiher questions remain unanswered. Firstly, we expected an overall reduction of IHS days and that this would have been accompanied by shorter length of stay. But length of stay, if anything, increased by 0.8 days ( 8.7 to 9.5 days). Presumably this increased length of stay is related to the increased proportion of IHS or it is related to the specific kind of patients who have to wait a long time till elderly homes or rehabilitation centers become available. Since the average length of stay in this hospital is under that of the other Dutch teaching hospitals, we have no reasons to assume that the situation elsewhere is better. Another issue is that improving a process for one department might lead to a change for the worse for another department. However, we have no indications for such a change. In reducing the number of inappropriate days of stay some ethical and practical problems may occur, such as whether a patient should be discharged and readmitted later or not. This means one has to weigh to pros and cons (extra planning efforts and patient inconveniences versus decrease of IHS). However, considering the strains on health care budgets, one quarter of inappropriate output is hardly affordable. 
Future research \& improvement actions

The use of 'Admission \& Discharge teams' should be extended to all wards in the hospital. At this moment, this is subject of discussion within several management teams and already several similar improvement actions are being implemented. However, there are no data on these improvement actions available yet.

Other hospital processes (i.e. application and performance of diagnostic procedures) have to be optimalized. Alternative approaches how to do so have to be designed, studied and implemented. This is a point of interest for the near future. Finally, a suitable solution for the problem of 'external IHS' has to be found. However, this will be a major challenge.

Acknowledgements

We would like to thank the reviewers at the wards for their efforts in the data collection. Without their hard work this study would not have been possible. 


\section{References}

1. Panis L, Verheggen F, Pop P. To stay or not to stay. The assessment of appropriate hospital stay, a Dutch report. Int J Qual Health Care, 2002; 14: 55-67.

2. Lorenzo S, Beech R, Lang T, Santos-Eggiman B. An experience of utilization review in Europe: sequel to a BIOMED project. Int J Qual Health Care 1999; 11:13-9

3. Lorenzo S, Lang T, Pastor R, Tampieri A et al. Reliability study of the European appropriateness evaluation protocol. Int J Qual Health care, 1999; 11:419-24

4. McDonagh M, Smith D, Goddard M. Measuring appropriate use of acute beds. A systematic review of methods and results. Health Policy 2000; 53:157-84

5. Gertman P., Restuccia J. The Appropriateness Evaluation Protocol: A Technique for Assessing Unnecessary Days of Hospital Care. Medical Care 1981; 9:855-71.

6. Restuccia J. Appropriateness Evaluation Reviewers' Manual. In: The AEP Final Report. Springfield, VA: National Technical Information Service, 1986, Publication No. PB87-1 12041.

7. Restuccia J. The evolution of Hospital Utilization Review Methods in the United States. Int J Qual Health Care 1995; 7:253-60.

8. Panis L, Gooskens M, Verheggen F. Pop P. Predictors of inappropriate hospital stay: a clinical case study. Int J Qual Health Care, 2003; 15: 57-66.

9. Smeets P, Verheggen F, Pop P. Panis L, Carpay J. Assessing the necessity of hospital stay by means of the appropriateness evaluation protocol: how strong is the evidence to proceed? Int J Qual Health Care, 2000; 12: 483-93.

10. Juran J, Gryna F. Statistical Process Control. In: Quality planning and analysis $3^{\text {nd }}$ ed. McGraw-Hill, New York 1993, ISBN: 0-07-112992-8.

11. Ingold B, Yersin B, Wietlisbach V, Burckhardt P, Burnand B, Bula C. Characteristics associated with inappropriate hospital use in elderly patients admitted to a general medicine service. Aging Clin Exp Res 2000; 12:430-8

12. Chopard P. Perneger T, Gaspoz J et al. Predictors of inappropriate hospital days in a department of internal medicine. Int J Epidem 1998; 27:513-9

13. Perneger T, Chopard P, Sarasin F et al. Risk factors for a medically inappropriate admission to a departement of internal medicine. Arch Intern Med 1997; 157:1495500

14. Dautzenberg P. Bremer R. Lormans, M Tanis J. Delay in hospital discharge in a general hospital. (in Dutch) Med contact 1997: 42:1310-3.

15. Vetter N. Inappropriately delayed discharge from the hospital: What do we know? BMI 2003: $326: 927-8$

16. Braveman P. Kessel W. Egerter S, Richmond J. Early discarge and evidence-based practice. Good science and good judgement. JAMA 1997: 278:334-6

17. Eriksen B. Forde O. Kristiansen I et al. Cost savings and health losses from reducing inappropriate admissions to a department of internal medicine Int J Tech assess Health Care 2000: $1147-57$

18. Kossovsky M. Chopard P. Bolla F et al. Evaluation of quality improvement interventions to reduce inappropriate hospital use. Int J Qual Health care 2002:3:227-32

19. Merom. D, Shohat T, Harari G, Oren M. Green M. Factors associated with inappropriate hospitalization days in internal medicine wards in Israel: a crossnational survey. Int J Qual Health care 1998: 10:155-62 
20. Menu-Branthomme A, Benamouzig R, Bejou B, Coste T, Rautureau J, Huet B. Inappropriateness of hospital days and causes of failure in a Gastroenterology and Internal Medicine ward. (in French) Gatroenterol Clin Biol 2002; 26: 29-37

21. Hall D, Bytheway B. The blocked bed; definition of a problem. Soc Sci Med 1985; 16: 1985-91.

22. Hermans E, Anten H, Diederiks J, Philipsen H. Long-term results and substitution among 'blocked bed' patients suffering CVA (in Dutch). Tijdschrift voor gezondheidswetenschappen $2000 ; 5: 275-9$. 

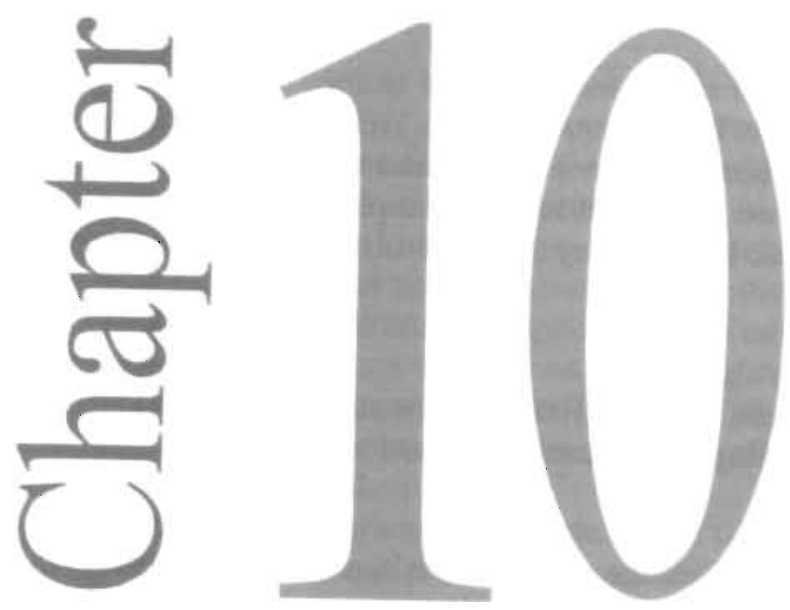

\section{The impact of hospital discharge proce- dures on hospital stay}




\begin{abstract}
Background.

Appropriate hospital stay should be: effective, efficient and tailored to the patients needs. Previous studies found that on average $20 \%$ of the hospital stay was inappropriate. Within Obstetrics inappropriate hospital stay (IHS) consisted mostly of delays in hospital discharge.
\end{abstract}

\title{
Objectives.
}

The specific goals of this study were: reducing IHS by fine-tuning the patient logistics; increasing the efficiency and providing a more comforting surrounding.

\section{Methods.}

New discharge policies using strict discharge criteria were implemented. Thereby, the mothers could leave the hospital in an earlier stage and use an external family accommodation, near the hospital.

\section{Results}

The total IHS decreased from 13.3 to $7.2 \%$. The delay in discharge procedures halved. P-charts showed a decreasing fluctuation in the IHS, indicating the current process to become more stable.

\section{Conclusions}

A significant reduction of IHS by the implementation of innovative hospital discharge policies was found, indicating a higher efficiency and accessibility of hospital services:

Keywords: P-charts, inappropriate hospital stay, IHS, hospital discharge, efficiency. 


\section{Introduction}

Many births occur without hospitalization in the Netherlands, either at home or in outpatient clinics, in the care of a community midwife. This midwife also monitors the pregnancy. If there is any indication for complication, the mothers-to-be are referred to an obstetrician. The outcome of planned home births is at least as good as that of planned hospital births. ${ }^{1-2}$ Nowadays approximately one-third of all deliveries in the Netherlands take place at home. ${ }^{3}$ Beside these births at home, deliveries can take place in an outpatient clinic. Without complications, the mother and baby can leave the hospital the same day. Although there are no official figures for the proportion of these deliveries, it is estimated to be approximately $15 \%$ of all deliveries. ${ }^{4}$ After birth the mother and baby are cared for at home under supervision of a district nurse and with the aid of a maternity assistant for about 10 days.

Only high-risk and otherwise difficult deliveries take place in hospitals under the supervision of an obstetrician. If there is a possible risk for the newborn, the mother-fo-be patient is referred to a neonatal center where -if indicated- the newborn can be admitted to a neonatal intensive care unit (NICU). If the newborn has to be admitted, specialized or intensive hospital care is required. For this reason the stay of the newborn is appropriate (necessary). In this case the mothers often have no medical indication for further hospital stay. Here the further stay of the mother can be labeled as 'inappropriate'. ${ }^{0.7}$ This stay obstructs efficient use of the hospital beds in the maternity ward and since the capacity of the obstetric wards and / or NICU is limited, some patients cannot be admitted and have to be referred to other centers. Therefore, in terms of economics or patient logistics, these patients should be discharged from the hospital. ${ }^{8}$ However, staying close to the new baby is very important for mother and child for different reasons. Beside this factor, some Dutch medical insurance companies do not cover expenses of maternity care at home as long as the baby is admitted to a hospital. Or expenses are only partly covered or covered for a limited period. For these reasons, the mothers of newly born children stay longer in the hospital than medically required.

Previous studies ${ }^{2,9-12}$ at the university hospital of Maastricht on the appropriateness of hospital stay showed that on average over $20 \%$ of the total hospital stay was inappropriate. Within the specialty of Obstetrics the proportion of inappropriate stay was relatively low $(13.2 \%)$. Nevertheless, this inappropriate stay accounted for approximately 800 days of stay annually. In addition, this inappropriate hospital stay (IHS) was mainly related to delay in discharge procedures (i.e. internal hospital procedures) $(94 \%)$. Therefore, interventions to reduce this inappropriate hospital stay were indicated.

The specific aims of this study were to evaluate the impact of alternative discharge procedures on IHS and to reduce IHS for mothers of newly born children. In this article we present data on the appropriateness of hospital stay in obstetric patients and the effect of altered discharge procedures on the appropriateness and the length of hospital stay. 


\section{Methods}

\section{Setting}

This study was conducted at the university hospital of Maastricht (azM). This 715-bed hospital has both a top reference and a district function and is located in the southeastern part of the Netherlands within an urban area.

\section{The assessment of IHS}

We used the Dutch Evaluation Protocol (DAEP) to assess the appropriateness of hospital stay. This instrument is based on the US medical-adult Appropriateness Evaluation Protocol ${ }^{13-14}$, adjusted and validated for use in the Dutch health care system. ${ }^{7.12}$ It consists of 19 generic (diagnosis-independent) and explicit (care related) criteria for appropriate hospital stay and a list of reasons for inappropriate stay. The DAEP assesses the appropriateness of the setting in which the care is provided, not the appropriateness of the care itself. ${ }^{15}$ Appropriate hospital care was defined as: 'Inpatient care, requiring continuous and active medical, nursing or paramedical treatment, which could not be provided through extramural care, daycare or outpatient care with regard to existing legislation.'

\section{Interventions to reduce inappropriate hospital stav}

Previous studies showed that the main reason why mothers remained hospitalized without medical need was the admission of their baby. ${ }^{7}$ This was especially a problem for families living at a long distance from the hospital. Another reason was the lack of maternity care at home once the baby was admitted to the hospital. The specific aim was to reduce IHS for mothers of newly born children. This had to be achieved by:

- Fine-tuning patient logistics by continuous feedback on IHS data and consultation on capacity / accessibility of accommodation

- Increasing efficiency by $20-30 \%$ without additional costs.

- Provision of adequate maternity care in a more comforting surrounding

- Hospial discharge in an earlier siage.

Therefore, new discharge procedures were implemented on the maternity ward.

The DAEP-criteria were used to assist in determining the day of hospital discharge. The discharge policies implied the use of strict discharge criteria, related to the DAEPcriteria. As an intervention, new discharge policies were developed and implemented. By these new criteria the mothers were discharged from the hospital in an earlier stage and transferred to an existing external family accommodation (Ronald McDonaldhouse), near the hospital. The required maternity care was provided by the hospital. Continuous feedback on the appropriateness of the patient stay was provided to the clinicians. The interventions were related only to the setting of this care, not the care itself. The appropriateness of patient admissions was not to be assessed. Data on IHS of Gynecologic patients were used to compare base line characteristics. Within this patient group, no alternative discharge procedures were implemented.

\section{Study sampling and data collection}

The study was set up as a retrospective cohort study. During a two-year period data on IHS were collected. During this period the changes in IHS were monitored by the cyclic use of the DAEP by registered nurses on a regular basis (i.e. every semester, the appropriateness of the hospital stay of every patient present was assessed during two weeks). These data on IHS (proportion of and reasons for inappropriate stay) were combined 
with general data from the hospital information system. The data were collected at the maternity ( 17 beds, +/- 1250 deliveries annually, nursing staff: 15 full time equivalents (fte)) and the gynecology ward (24 beds, +/- 1000 admissions annually, staff: $17 \mathrm{fte}$ ).

\section{Statistical analysis}

Descriptive analysis was applied to the data concerning the proportion of and the reasons for inappropriate hospital stay. For the comparison and the significance of differences of the baseline characteristics within and between patient groups the Student's ttest or the Pearson Chi-square test were used (where appropriate). All data analyses were performed using the SPSS-PC software package, version 10.0.

\section{Results}

During the study (November 2000 to October 2002), a total of 2889 days of hospital stay of gynecologic and obstetric patients have been assessed (table 1). The proportion of IHS within obstetric patients decreased from $13.3 \%$ in 2000 to $7.2 \%$ in 2002 . This decrease was statistically significant $(\mathrm{p}<0.025)$. Simultaneously, the average length of hospital stay (LOS) of obstetric patients increased by 0.7 days. Within gynecologic patients the IHS almost quadrupled ( $7 \%$ to $26 \%$ ), while the LOS remained the same.

Table 1: Study sample

\begin{tabular}{|c|c|c|c|c|}
\hline Year & 2000 & 2001 & 2002 & Total \\
\hline \multicolumn{5}{|l|}{$\begin{array}{l}\text { Maternity ward } \\
\text { Days of stay }\end{array}$} \\
\hline Appropriate & 471 & 372 & 384 & 1227 \\
\hline Inappropriate & $72(13.3 \%)$ & $64(14.7 \%)$ & $30(72 \%)$ & 166 \\
\hline Length of stay & 2.9 & 3.3 & 3.6 & \\
\hline \multicolumn{5}{|l|}{$\begin{array}{l}\text { Gynecology ward } \\
\text { Days of stay }\end{array}$} \\
\hline Appropriate & 546 & 448 & 306 & 1300. \\
\hline Inappropriate & $42(7.0 \%)$ & $44(8.9 \%)$ & $110(26.4 \%)$ & 196 \\
\hline Length of stay & 5.3 & 4.7 & 5.0 & \\
\hline Total & 1131 & 928 & 830 & 2889 \\
\hline
\end{tabular}

'Pearson Chi-square 7.411, df 2, p 0.025, Pearson Chi-square 58.526, df $2, p=0.001$

Initially the IHS in the obstetric ward consisted mainly ( $94 \%$ ) of delays in discharge procedures (fig. 2). These delays were caused by the hospital stay of 'healthy' mothers who did not need further hospital stay, while their baby had to be admitted to the hospital. At the end of this study only half the IHS $(46 \%)$ was related to discharge procedures (fig 2). The remaining inappropriate stay was related to delays in hospital procedures, such as delay in diagnostic or therapeutic interventions.

Within the group of gynecologic patients, data showed no difference in the IHS related to discharge procedures. The significant increase in IHS was related to delays in hospital procedures, patient characteristics (e.g. refusal) and the unavailability of other health care facilities -outside the hospital-, so the patient could not be discharged. 
Using P-charts to monitor the IHS, within the maternity ward a decreasing fluctuation in the IHS was found during this study, indicating the process to become more stable. The P-charts for the gynecology ward showed more fluctuations over this period, demonstrating the process to become less stable and therefore less predictable.

Figure 1: Evolution in hospital stay of obstetric patients

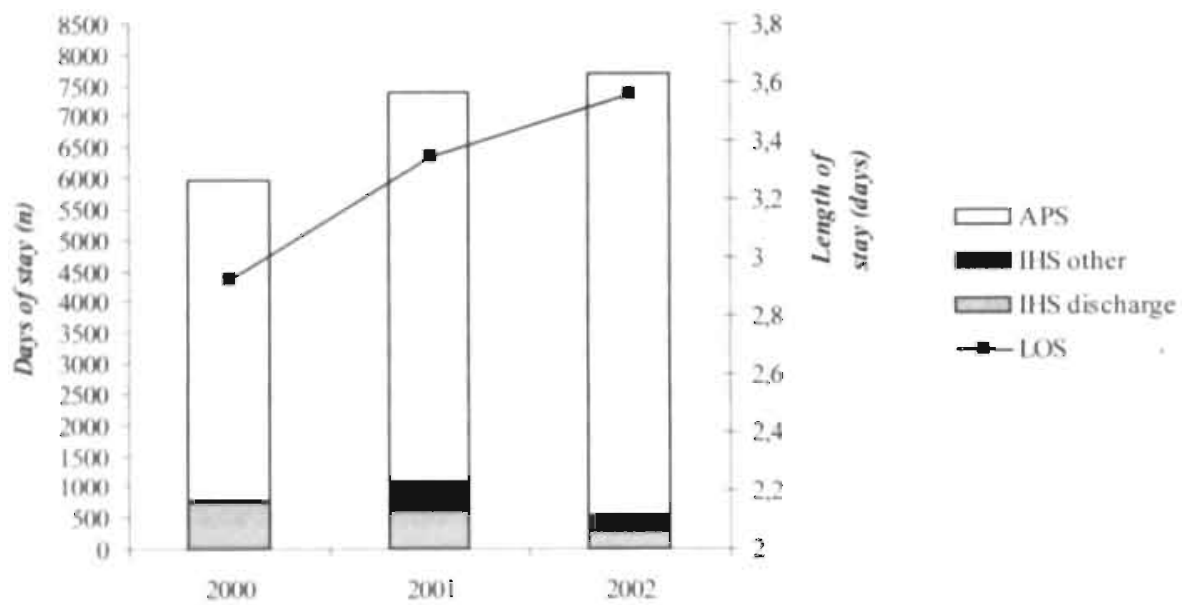

APS: Appropriate patient stay, IHS: Inappropriate hospital stay, LOS: Length of stay

The number of admissions of obstetric patients increased from 2047 to 2213 per year ( 8 $\%$ ), while the admissions to the gynecologic ward decreased from 1406 to $1344(4.5 \%)$ (p: .012), the average length of hospital stay (LOS) increased in Obstetrics ( 0.6 days) while it slightly decreased in Gynecology (0.2 days) (Fig.1). The increase in admissions to the obstetric ward was related to the growing focus on high-risk and / or difficult deliveries, admitting these patients earlier to the hospital. This explains the increase in LOS during this period (fig. 1). At the same time, the implementation of new discharge procedures (earlier discharge or discharge to family accommodation) resulted in a better throughput, so more patients could be admitted.

Within the gynecology ward the treatment of oncological patients was emphasized. leading to prolonged in-patient care. Also no new discharge procedures were implemented. This resulted in less patients while the overall LOS slightly increased. 


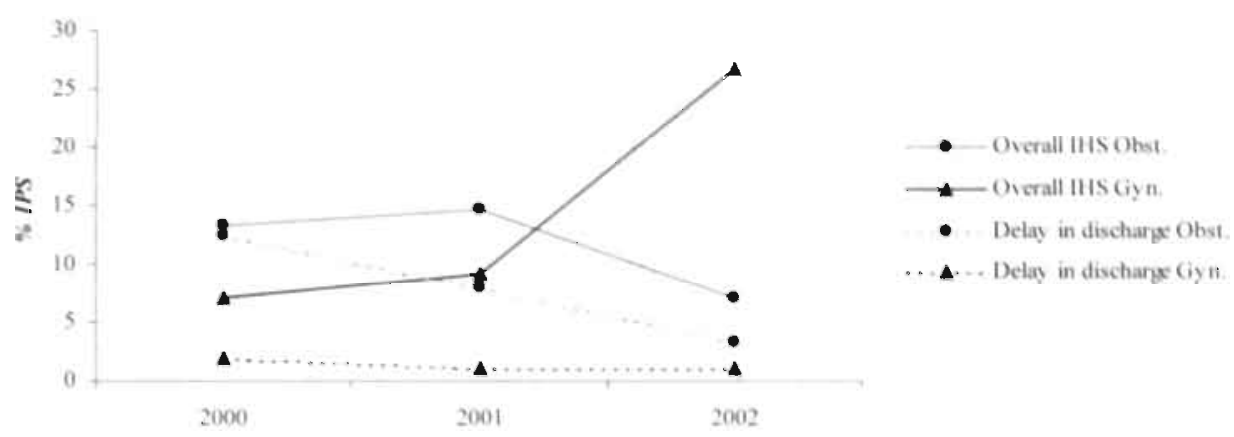

IHS: Inappropriate hospital stay

\section{Discussion}

The majority of deliveries in the Netherlands occur in a home or outpatient situation. Although internationally there is a growing attention to humanizing and demedicalizing childbirth and initiatives have been taken to promote a humanized approach, deliveries involving an increased risk for mother and / or child still musi take place in a clinical setting. In this study we showed that is possible, to shift the care for mothers of a newborn child -if there is no further risk for the mother- to an outpatient setting, on the condition of good alternative care. In this way the proportion of IHS due to hospital discharge procedures decreased by almost half and the overall proportion of IHS in the maternity ward decreased significantly avoiding several hundreds days of hospital stay. Within gynecologic patients however, the proportion of IHS due to discharge procedures remained the same while the overall proportion of IHS more than doubled. There are some plausible explanations for these evolutions in IIIS:

Although, the reduction in IIIS in Obstetrics could was realized by the use of strict discharge policies, these policies only work if adequate maternity care is available. The fact that there are no readmissions might reflect the quality of this care. Within obstetrics we were able to guarantee adequate maternity care, so the mother could leave the hospital in an earlier stage. Beside this, these mothers are young and are mostly able to care for themselves. And since baby's charmi people a new mother can rely almost always on the help of friends or family. This facilitates an early hospital discharge. The shift of maternity care is only possible if there is sufficient capacity available in the outpatient setting. If this capacity is fully in use, the shift comes to a temporarily standstill and the mothers remain hospitalized, and the IHS will increase again. 


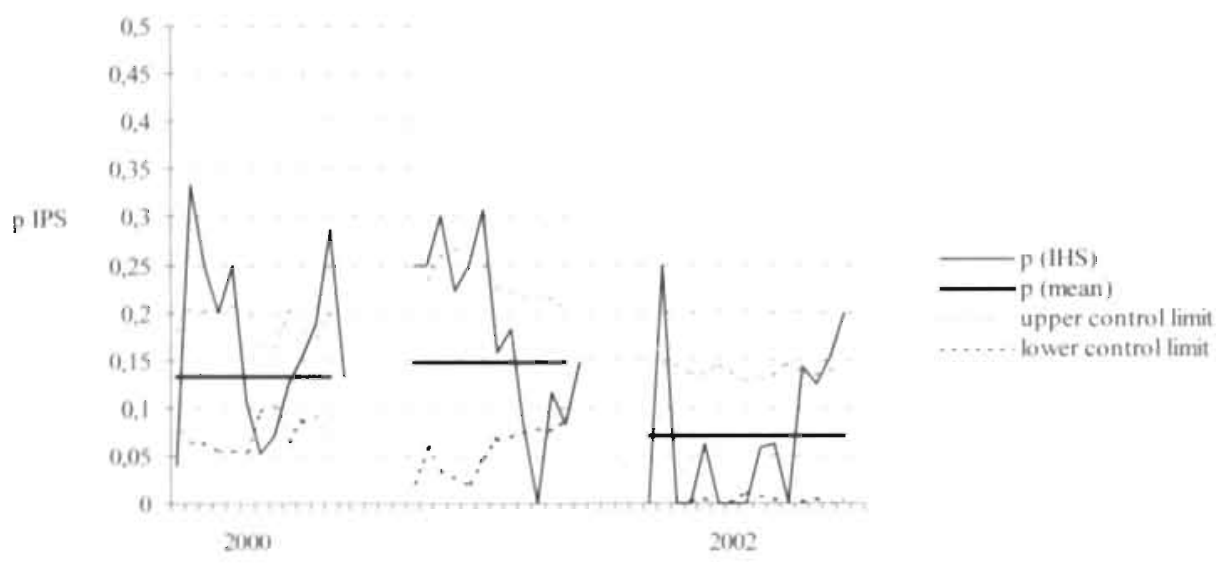

Figure 5: P-chart IHS Gynecology

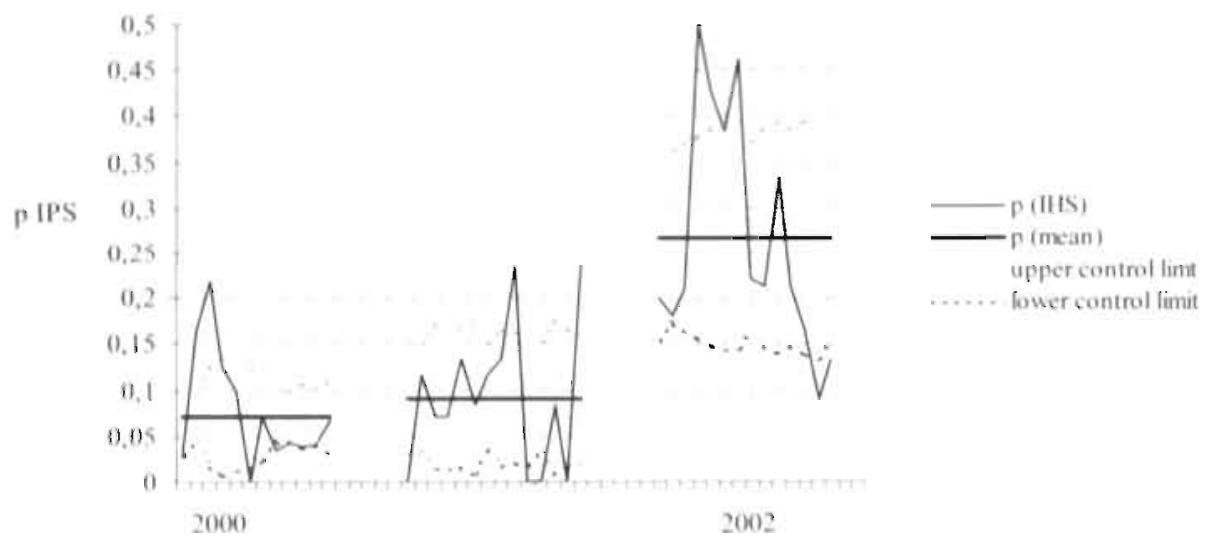

Within the group of gynecologic patients extra attention was paid to improve discharge procedures. This did not decrease IHS related to hospital discharge procedures. At the same time the medical specialists focused more on surgical interventions in oncology patients. This resulted in more procedures (diagnostic or therapeutic), more interventions and more waiting time required for these procedures. Maybe for this reason, the selection of gynecological patients was not suitable as control group. But nevertheless, if more procedures are requested, the planning of these procedures should be efficient as possible, so IHS is reduced to a minimum. On the other hand, these patients are often elderly, very sick or disabled patients, which cannot take care for themselves or cannot rely on volunteer aid due to their disabilities. So the department can arrange their processes to an optimum. if there is no care available outside the hospital, the patient has to remain hospitalized. ${ }^{16}$ Nevertheless, efficiency can be improved by optimalizing hospital processes without additional costs. 


\section{Conclusion}

Shifting maternity care from the hospital to an outpatient setting, providing adequate care, can be a useful alternative to decrease hospital stay. This study showed that the use of strict discharge criteria can reduce IHS related to hospital discharge procedures. It also shows that an increase in efficiency is possible without additional costs. Within gynecological patients the IHS related to hospital discharge procedures remained the same, while the overall inappropriate stay almost quadrupled. Waiting time problems as a consequence of diagnostic and therapeutic procedures and interventions for more oncological patients admitted to the hospital were the reasons. For these problems specific improvement actions focusing on the planning of procedures will be set up in the near future. The fact remains that a shift of a patient to an outpatient setting depends on the available capacity of extramural facilities. 


\section{References}

1. Wiegers T, Keirse M, Van der Zee J, Berghs G.Outcome of planned home and planned hospital births in low risk pregnancies: prospective study in midwifery practices in the Netherlands.Br Med J 1996; 313:1309-1313.

2. Wiegers T. Home birth or hospital birth: a prospective study of midwifery care in the Netherlands. Eur J Obstet Gynecol Reprod Biol 1998; 79:139-141

3. Statistics Netherlands: Statline, the statistical database on the Netherlands cited 2001 Jul 18. Available from: URL http://argon2.cbs.nl/statweb/index_ENG.stm.).

4. Shirm E, Tobi H, de Jong-van den Berg L. Low use of medication in home deliveries in the Netherlands. Int J Gyn Obst. 2002:79: 5-9

5. Page L. Human resources for maternity care: the present system in Brazil, Japan, North America, Western Europe and New Zealand. Int J Gynecol Obstet 2001; 75: S81-8

6. Payne S. Identifying and managing inappropriate hospital utilization: a policy synthesis. Health Serv Res 1987; 22(5): 709-69.

7. Panis L, Verheggen F, Pop P. To stay or not to stay. The assessment of appropriate hospital stay, a Dutch report. Int J Qual Health Care, 2002; 14: 55-67.

8. Lequien P. Reduction of the length of stay of mothers at the maternity unit (in French) Arch Pediatr, 2001; 8 suppl 2: 487-8

9. Smeets P. Pop P, Verheggen F. Assessing the appropriateness of hospital stay: the possibilities of the appropriateness evaluation protocol (in Dutch). Medisch Contact 1997; 52: 757-60.

10. Smeets P, Pop P, Verheggen F. Assessing the appropriateness of hospital stay: features of quality assurance and utilization review tools (in Dutch). Medisch Contact $1997 ; 52: 760-61$.

11. Smeets P, Verheggen F. Pop P. Panis L, Carpay J. Assessing the necessity of hospi= tal stay by means of the appropriateness evaluation protocol: how strong is the evi= dence to proceed? Int J Qual Health Care, 2000; 12:483-93.

12. Panis L, Gooskens M, Verheggen F. Pop P. Predictors of inappropriate hospital stay: a clinical cose study. Int J Quai Health Care, 2003; 15:57-66.

13. Gertman P. Restuccia J. The Appropriateness Evaluation Protocol: A Technique for Assessing Unnecessary Days of Hospital Care. Medical Care 1981; 9:855-71.

14. Restuccia J. Appropriateness Evaluation Protocol Reviewers' Mamual. In: The AEP Final Report. Springfield, VA: National Technical Information Service, 1986, Publication No. PB87-112041.

15. Lavis J, Anderson G. Appropriateness in health care delivery: definitions, measurement and policy implications. Can Med Assoc 1996; 154:321-8.

16. Murphy M. Noetscher C. Reducing hospital inpatient lengths of stay. J Nurs Care Qual, 1999; Nov: 40-54. 

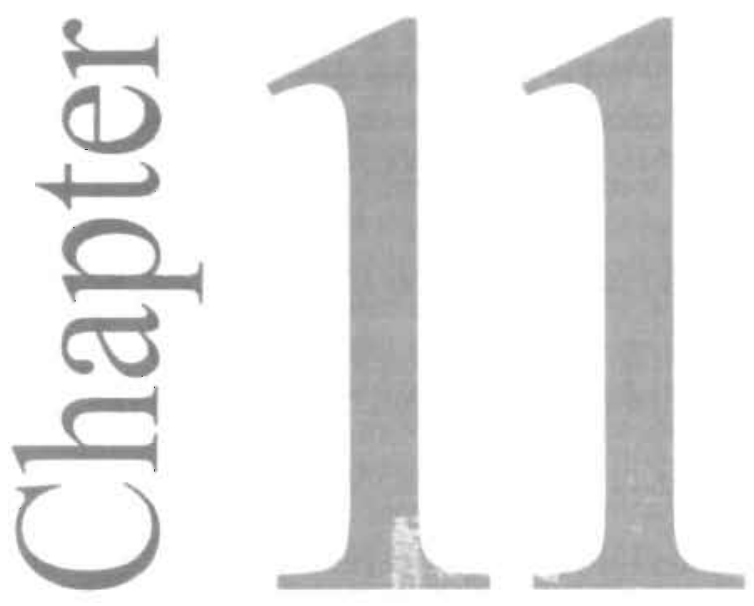

\section{General discussion}

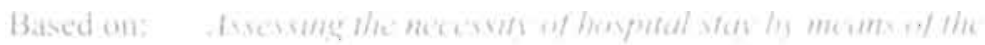

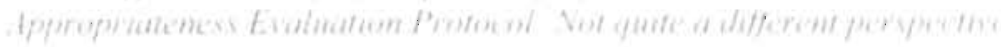

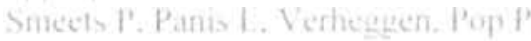

Int J Qual Health (are 2001: 13:345-6

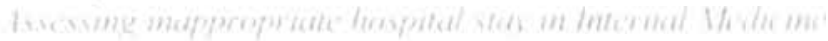

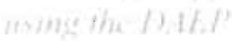

Punis I. Vethegean I Pup P. Pritis M

Int .I Qual Health Care 2001: 14: 431-2 
In this thesis, the central research objective was to assess the appropriateness of the patient's stay in an acute care hospital. For this purpose a measurement instrument was developed and evaluated for validity and reliability. All assessments were performed in the University Hospital of Maastricht (azM). Particularly, the following questions have been answered:

1. 'What is appropriate hospital stay (IHS) and which factors affect it?' (chapter 2 \& 3)

2. 'How can we assess IHS?' (chapter 4)

3. 'What kind of measurement instrument do we need?' (chapter 5)

4. 'Can IHS be identified within specific processes or patients?' (chapter 6 \& 7)

5. 'Is IHS predictable?' (chapter 8)

6. 'Which interventions are needed to reduce IHS?' (chapter $9 \& 10$ ).

This final chapter focuses on the relation between the different answers we have obtained and reflects on the implementation of specific actions to reduce IHS. These actions can be seen as the result of a quality improvement cycle that identified a problem, attempted to correct the processes responsible for it, and evaluated the results of improvement.

\section{Changes and improvement}

The core business of health care is to provide the right care at the right time. ' Ideally, this high quality care should be provided with the highest possible degree of efficiency. Only then, the best possible care is rendered, for a given budget. A day of care in an acute care hospital, without (para)medical interventions that justify this stay, can be regarded as a form of inefficiency (i.e. inappropriate hospital stay). Indeed, delays are common in health care. And although these delays are often regarded by patients and providers as a part of the care process, they can also be seen as a possibility for quality and efficiency improvement. Improvement requires change, but change is not always an improvement. Quality and efficiency improvement can be assisted by a systematic approach of planning, implementation, control and (re-)action (fig. 1).

Fig. I: Deming or improvement cycle

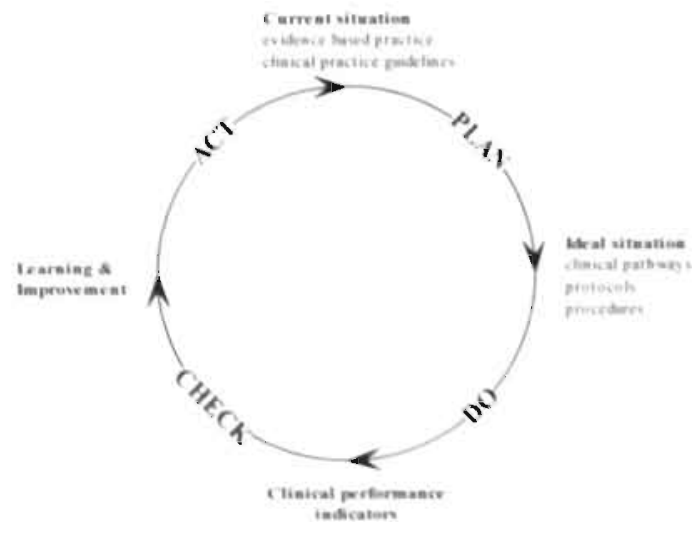




\section{Concepts and measurement of appropriateness}

\section{Appropriate health care setting}

Although there may be different views about inappropriate health care delivery, health care that does not benefit the patient should be avoided. Additionally, if care is required it is inefficient to provide it in an inappropriate setting. An inappropriate setting means that care could be provided in an alternative setting at lower costs (usually on an outpatient basis, in other health care facilities or at home) (chapter 2). However, the verb "could" should be emphasized; the required care may not be available in an alternative setting. For example, a patient may not be sick enough to justify appropriate hospital stay but might be too sick to be sent home without adequate home care. If the home care services are unavailable, then hospital admission may theoretically be inappropriate, but still might be the best available option.

In the absence of alternative health care settings, the patient's interest (and preferences) should prevail. The assessment of IHS should not be regarded as a tool for the management of individual patients, but rather as a tool for utilization review, allowing management to redesign and optimize care processes. Hence, within this study, the availability of other facilities has not been taken into account for the assessment of IHS.

\section{Appropriate hospital stay}

Except for emergency admissions, every patient in the Netherlands has to consult a general practitioner or medical specialist before he or she can be admitted to a hospital. If a general practitioner is seen, this general practitioner consults a medical specialist for an opinion on whether an admission to a hospital is indicated. Only then, the patient will be admitted to a hospital. If a patient arrives at an emergency depariment of the hospital, the need for admission is assessed there. If possible, the patient is treated, or he is referred to another health care facility. This approach reduces the risk of inappropriate admissions. However, even appropriate hospital admissions can generate days of inappropriate hospital stay. In this thesis only the appropriateness of the hospital stay (the setting) has been assessed. Therefore, appropriate hospital stay was defined as: "inpatient stay, requiring continuous and active medical, nursing or paramedical treatment, which under existing legislation can not be provided through extramural care, day-care or outpatient care". This definition proved to be feasible in assessing IHS within the boundaries of this thesis.

\section{Inappropriateness vs, redundancy}

The question remains if inappropriate hospital stay (IHS) should be reduced per se (i.e. efficiency improvement) or that a reduced IHS should be put against the possible detrimental effects and means that are needed to reach the goals (i.e. cost - benefit analysis). Hence, the ultimate goal of a 'lean production' (zero IHS) may not always be the best option (chapter 2). IHS may function as a buffer to enable the optimal use of very expensive medical equipment. Also, there will always be other factors inducing IHS uncontrollable by the health care organization itself, e.g. the lack of available alternative care facilities or problems in the patient's family environment. Since hospitals must provide adequate care, they cannot discharge a patient, knowing that there will be no further care in the home situation. These inefficiencies might be considered transaction costs, needed to avoid much higher costs elsewhere in the health care setting. "Even if IHS is assessed and found, subsequent actions to reduce this IHS are not always obvious. But the danger lurks in 'over-optimalizing' appropriate use, so that appropriate use 
might result in underuse or even in misuse of care (e.g. closing hospitals leading to a decreased access to similar facilities). This is not in the interest of the patient since misuse or underuse has negative consequences for the quality of care and might harm the patient. As mentioned in the introduction, health care must be among other things, safe and patient-centered. ${ }^{6}$

\section{The assessment of inappropriate hospital stay}

Several studies depict the Appropriateness Evaluation Protocol (AEP) as a practical instrument to screen for inappropriate hospital stay (IHS) and its reasons. ${ }^{7.21}$ It also enables users to monitor the effect of feedback or other interventions. However, literature review (chapter 4) revealed some limitations in the AEP as a general instrument for assessing IHS. More direct methods focusing on changing test order behaviour and discharge planning could have a better acceptance and cost-benefit balance. But although these intervention programmes may be cheaper as such, their evaluation can be very costly as well. ${ }^{22.23}$ Hence, the assessment of the appropriateness of the health care seiting is still relevant. Hence, the assessment of the appropriateness of the health care selting is still relevant. ${ }^{24}$ Perhaps care process analysis and continuous monitoring by statistical process control may provide further opportunities for quality improvement. If a clinical process can be made transparent, and is not too complex, clinical performance indicators can be used to assess the quality of care and the appropriateness of the hospital setting. Also, the choice for more diagnosis or specialization dependent approaches -despite their inherent disadvantages- is still open. ${ }^{23-26}$ However, AEP-like instruments are useful and desirable for quality assurance or utilization review. Therefore, the development of an AEP that could be used in the Dutch health care setting was (-we considered-) suitable.

In this thesis, some data had to be excluded from the analysis due to incompleteness of these data (missing data). The studies in this thesis, assessing frequencies of IHS (chapter $5,6,7,9,10$ ) showed an average proportion of excluded data of $0.9 \%$ (range: 0 4.6). The ratings of inappropriateness in these studies are similar to each other and similar to other international studies. ${ }^{7.21}$ So, we can safely conclude that the bias caused by these missing data items will be minimal in these studies. In one study (chapter 8 ) onethird of the data $(32.5 \%)$ has been removed. These data concerned repetitive measurements concerning the same patients. Since the design of the study was to assess predictive factors of IHS, all data on patients with repetitive measurements during their stay had to be removed. This was done to avoid bias on gender, age, medical specialty, home situation, etc. Systematic removal of first or last days would have resulted in bias since the incidence of IIS is higher at the end of the hospital stay (chapter 8). Alternative statistical methods include the complicated method of boot-strapping. However, this approach was not chose since the study sample characteristics and the characteristics of the patients that were removed did not differ. In addition, the presented proportion of inappropriateness in this study was similar to the proportion of inappropriateness in the removed data as in literature. Hence we believe that it is unlikely that important biases occurred. 


\section{Lessons learned}

\section{The measurement instrument (DAEP)}

The Dutch Appropriateness Evaluation Protocol (DAEP) proved to be a valid and reliable instrument to assess the appropriateness of hospital stay and to detect the overuse of hospital resources. In daily practice its application is relatively easy and efficient in use. The data obtained by using the DAEP can support the design of specific quality improvement programs. Furthermore, it enables users to monitor the effect of feedback or other interventions with regard to the appropriateness of the setting of the care. Process analysis and continuous monitoring provide subsequent opportunities for quality improvement. If a clinical process can be made transparent, clinical performance indicators can be developed to assess and monitor the quality of care in general.

Yet the DAEP has some limitations. Since more and better medical technologies become available, there is a constant shift in care from a hospital setting to a day care or ambulatory setting. Therefore, it is necessary that the criteria, on which the assessment of appropriate stay is based, are evaluated on a regular basis. Moreover, there might always be a gray area in which it is not possible to assess the appropriateness of the setting (e.g. the continuous shift of patients to an ambulatory setting, due to new evolutions in treatment strategies or technologies). The instrument can also be misused, to alter ratings of inappropriateness. However, if the instrument is linked to the general patient information system of the hospital, the plausibility of these ratings can be checked afterwards. Other limitations might be that the assessment of inappropriate hospital stay may be perceived as a check on the appropriateness of the provided care itself or as a check of professional performance. Professionals might become disappointed if the instrument identifies problems that cannot be influenced immediately (e.g. waiting lists for nursing homes).

\section{Inappropriate hospital stay}

In this thesis several bottlenecks in care processes within our hospital have been pointed out (chapter $5,6,9,10$ ). These problem areas are related to delays in the care processes inside or outside the hospital. The main causes of 'internal delays' were delays in diagnostic and / or therapeutic procedures and delays in hospital discharge. Both types of delay can be linked to the lack of written procedures (e.g, checklists, scheduling of critical pathways) and the lack of specific process analysis / flowcharting (e,g. waiting times or adverse patient occurrences). The lack of written procedures, describing each step of the logistic process of the patient and the time involved, leads to poor control of this process.

'External delays' (i.e. unavailability of other health care facilities) cannot be directly controlled by the organization. Nevertheless, this type of IHS remains a major problem. The so-called 'blocked beds', ${ }^{27.28}$ are a good example. These patients are staying in the hospital without medical indication, but need care that is not available in the home situation. These patients are just waiting for a transfer to an other, not yet available, care facilities. Extra attention has to be paid to this specific type of IHS, since creative solutions in reducing this type of IHS have the potential to free a substantial portion of hospital beds and thus improve the throughput. 


\section{The patient}

Patient characteristics are useful in predicting IHS. As the age of the patient increased, the IHS rates showed an upward trend (chapter 8). The availability of home care affected. IHS rates in a positive way. Also the IHS appeared to be linked to the medical specialty. The availability of a clear medical policy or clinical pathways reduced the risk of IHS. ${ }^{29-31}$ If a patient matches one or more of these characteristics extra attention must be paid in avoiding IHS, focusing on the planning of and the compliance with therapeutic, diagnostic or discharge procedures. But the patient's wishes and preferences must always be kept in mind. ${ }^{32-35}$ During the design and validation phase of the DAEP, the departments that participated in these studies were divers (chapter $4 \& 5$ ). Later on, during the intervention phase, patient groups were selected based upon a high proportion of IHS and the potential for improvement (chapter $9 \& 10$ ).

\section{Interventions to reduce inappropriate hospital stay}

During the patients' stay. IHS can occur during any stage of the treatment. Our study (chapter 8) shows that IHS is concentrated in two stages. The first stage answers the demand for, or the performance of diagnostics and / or therapy. The second stage is related to the patient's discharge from the hospital. We tried to reduce both types of IHS. In two studies (chapter $9 \& 10$ ) we were able to improve the process of hospital discharge. Here the implementation of specific procedures on hospital discharge proved to be successful. Also the transfer of patients to available altemative setting showed to be very useful (chapter 10 ). We also tried to reduce IHS related to delays in diagnostics (chapter 9). Unfortunately, no significant improvement for this type of IHS was found in this study. There are several possible explanations for this. Most probably, the improvement strategy was insufficient; the use ot 'slots' might be usetul, but to tew 'slots were created within this improvement effort.

\section{Future perspectives}

\section{Delass in procedures (patient flow)}

Few care processes are designed to achieve optimal flow for patients, Noninterchangeable resources, such as operating rooms and diagnostic facilities, tend to be major bottlenecks. Redicing delays depends on assessing and improving flow throughotit the entire system, even from an inpatient setting to long-term care facilities. The key to this lies in reducing process variation that impacts flow. While some variability is nomal, excessive variation is not and should be eliminated. When delay occurs, patients and providers are affected across the continum of care. Waiting and delays are not the result of lack of effort or commitment of the staff and working harder cannot solve these problems. This is illustrated by what D. Berwick (CEO of the institute for Healtheare improvement) calls the first law of improvement: "...Every system is perfectly designed to achieve the results it achieves..." ${ }^{36}$ Hence, the improvement of flow needs a redesign of the overall process that creates the flow problems. Optimal care can only be delivered when the patient is in the right place with the right provider and the right information at the right time. Improvement efforts in hospitals show that it is possible to reduce delays in hospital care, improving the flow of patients throughout the care system. Improving flow depends on understanding the variability throughout the hospita! system. This improvement should focus on the variation in delays that occur when capacity does not match demand. If the degree of variation in the demand exceeds the capacity to absorb that demand, it will result in delays. Predicting and managing 
variability to allocate resources appropriately can improve the efficiency of processes, patient outcomes and reduce costs. Three steps must be taken: evaluating patient flow, measuring and understanding flow variation and improving flow.

\section{- Evaluating patient flow}

It has to be determined how often patient are moved through the system in a timely and efficient manner. Here, the frequency of "blocked beds" and hospital occupancy can be used as indicators. Possible solutions are: working to reduce flow variation and "extending the chain": working with others along the continuum of care, including those outside the hospital, to smooth the flow of patients into and out of the organization.

- Measuring and understanding flow variation

Variation is inherent in health care. It results from clinica! variability (patient's condition), flow variability (patient's arriving), and professional variability (provider's skills). It is suggested by E. Litvak that the following scenario would eliminate variability: ${ }^{38}$

- All patients have the same disease with the same severity:

- Patients arrive at the same rate every hour:

- All providers (physicians and nurses) are equal in their ability to provide quality care.

Some kinds of variability ("random variability") cannot be eliminated; they must be managed, e.g. the patient's syndrome or his arrival (patient variability). Other types of ("non-random") variability can be driven by individual preferences rather than actual demand. Non-random variability should not be managed; it should be eliminated. Volume and occupancy rates are often calculated and displayed as means or averages. However, it is the variation in these measurements that causes most of the flow problems in hospital systems.

- Improving flow

Surgical and diagnostic schedules are the major source of variation in how. Programs that are entirely filled leave little flexibility for emergencies. If the unschede uled procedures averages 10 percent or more, adequate space should be left in the schedule otherwise the organization will routinely experience delays for some patients. ${ }^{38}$ Since a majority of procedures is scheduled, most of the facilities should be assigned. Utilization of the scheduled rooms then becomes more predictable and controllable, and waiting times for unscheduled procedures become manageable. Concerns about the cost of designating personnel strictly for emergencies are unwarranted; since these costs are outweighed by the costs of canceling and delaying scheduled procedures when emergencies disrupt a day's elective schedule. Furthermore, since most surgical admissions are pianned in advance, the corresponding diagnostic procedures should be carried out as much as possible on an outpatient basis. This would avoid IHS related to diagnostic procedures. Subsequently, the surgica! procedure should be performed on the day of admission (same day surgery). Al-though at this moment diagnostic procedures before surgery are being performed more and more on an outpatient basis, it could be extended to all elective patients. ${ }^{39}$ At this moment only emergency diagnostics are performed outside regular business hours. If diagnostic facilities were used outside business hours, these hours could be reserved for other (in-patient, non-urgent) applications. Mathematically, this could increase capacity by $200 \%$ (i.e. 24 vs. 8 hours). However, to do so would require fundamental changes in the organization and funding of the Dutch health care sys- 
tem. Furthermore, this is still an emotive subject. Another possible solution is the socalled 'Independent Diagnostic Center'. ${ }^{40}$ These centers are specialized in performing specific high quality diagnostic tests and can provide extra capacity at an economic rate. As a possible solution to avoid waiting for diagnostics, the criteria for the use of certain diagnostics could be tightened and the subsequent compliance increased. This would mean less, but more rational use of hospital facilities. In addition, critical evaluation of the application for diagnostic procedures must be performed in order to give priority to urgent applications and to discourage unnecessary diagnostics. As a last option, additional equipment could be installed, although this option would increase costs.

Since hospitals must have some slack in their system (e.g. overcapacity for emergency reasons) it is impossible for them to charge a break-even price. Yet, according to Pareto's rule, ${ }^{41} 80 \%$ of the total delay will occur in only $20 \%$ of the patients. Special attention has to be paid to these patients with a high probability of IHS. Their logistic process and its time schedule must be monitored continuously. For the remaining $80 \%$ of the patients, pathways for interventions with a low risk of complications or inconveniences must be identified and set up, in order to smoothen the flow of the hospital processes, to speed up their hospital discharge and to decrease the length of hospital stay.

Specific process analysis and flowcharting will provide insight in the effective application or performance of hospital procedures. But if decreasing IHS seems to be a matter of resourcing and logistics, what kind of procedure needs to be planned at what time, which operational policies and patient logistics have to be altered? A cost-benefit analysis should be made in order to select the most efficient patient logistics. Furthermore, existing comorbidity might require extra observation in a hospital setting to eliminate possible complications.

\section{Delass in hospital discharge}

The patient's discharge process in the hospital is often designed inefficiently. In general, output (and throughput) relies on the timely delivery of a product. The existence of wating lists, etc. is often caused by blockage at the end of the process: the discharge procedure. New patients can't be admitted until beds open up, and beds don't open up until all the steps of the process are completed. Often, there are patients who ware medically ready for discharge, but some necessary steps have not been completed e.g. a transfer letter of a recipe not written or the patient's education is incomplete.

Eleciive patients can be adinitted according to planned schedules. Although the date and time of hospital discharge may be uncertain for some patients, health care providers can usually predici one day in advance which patients can be discharged the following day. ${ }^{42}$ However, the prediction of discharge after two or more days is increasingly less accurate. So, the knack is to match discharges to the admission pattern, with enough time to prepare for the next patient. This can be achieved by scheduling the discharge: assigning patients to a day and time for the discharge to occur and planning all necessary activities that have to be completed before this discharge. Each day the discharge date is reaffirmed and planned activities are performed accordingly. If the discharge process is slowed down for any reason, the question is asked: "What must be done today to meet the scheduled day of discharge?" Hence, the correct designated "pathway" for that patient is designed. "Such designated pathways should be developed for each of the major diagnostic categories that are being seen in a care unit. Observing and documenting 
discharges over time in these major diagnostic categories can not only be used to design these pathways, but also monitor adherence to the pathways.

In summary: discharge procedures should already start at the patient's admission. ${ }^{44-47}$ In this way, the patient can be discharged immediately from the hospital at the moment there is no further need of hospital stay. This means that the patient discharge is a service that can be predicted and scheduled.

\section{Inappropriate hospital stay due to 'external factors}

The hospital cannot directly control external factors influencing hospital stay. Nevertheless, inappropriate stay due to the unavailability of other care facilities remains a major problem. The set-up of a "care-hostel", age related geriatric services or extended home care ${ }^{28}$ might provide useful solutions. These services can help the patient to recover outside a hospital setting and result in a more appropriate use of hospital resources. Since for example patients education and physical training can potentially be performed in these facilities. Some inappropriate days of stay due to internal reasons (e.g. delay in discharge) might also decrease, by the use of such facilities. Working with general practitioners and long-term care facilities is an effective strategy to improve patient flow. Promoting advanced access scheduling (sometimes referred to as "open access") ${ }^{4 k}$ in physicians' offices can stimulate timely access to ambulatory care in an appropriate setting, rather than resorting to the hospital, and working with hospice services can assure that end-of-life care is provided in the most appropriate, but least intensive, setting. Although both problems should be tackled, the issue to be considered is: "What is more cost-effective; arranging extra capacity of extramural health care facilities or accepting the existence of (some) IHS within an acute care hospital?". Alternatively, the creation of units that provide hostel type of care within hospitals might be considered.

\section{Consequences of the reduction of inappropriate hospital stay}

\section{Potential adverse effects}

The reduction of IHIS should be seen in the perspective of its consequences. Sometimes patients might experience a worse outcome from a shorter stay or vice versa. ${ }^{40}$ For some procedures there is a theoretical- minimal period during which hospitalization would be desirable. This period is mainly determined by the time needed to recover adequately or the need for observation or monitoring before the risk of complications is sufficiently reduced to enable a safe discharge. Hospital care may also protect patients from onerous household chores or adverse home circumstances. Although it is not expected that an early discharge wil! lead to more complications or discomfort for the patient ${ }^{52}$, supplementary facilities, such as the back up of home care, should be arranged if patients are discharged earlier from the hospital. Proper facilities or services should be available, if any complications after discharge occur in the home situation. However, some complications labeled in literature as minor; including chest and wound infection, haematoma, unexplained fever, thrombophlebitis and wound discharge, are hardly viewed as minor by patients. ${ }^{3}$ And the possibility exists that patients with a shorter hospital stay may be shown to be less satisfied. 


\section{Potential benefits}

Reduction of IHS might result in reduced health care costs from a national perspective. However, from the hospital perspective, a shorter stay, without IHS, can lead to a larger expenditure per day of care. This is because the highest costs usually occur in the first half of the stay when input from staff, investigations, and interventions are at a maximum. Days of IHS occur mostly in the second half of the stay where the patient is less dependant and care is less intensive. ${ }^{54}$ Hence, while the reduction of IHS enables a hospital to use its capacity more efficiently in order to treat more patients, the current system of reimbursement does not cover the increased overall hospital costs. This might change when the Diagnosis Treatment Combination system (in Dutch: Diagnose Behandel Combinatie, DBC) is fully operational. The more efficient, use of hospital capacity may have a positive effect (i.e, reduction) on waiting time and waiting lists. Little research has focused on overall costs and benefits of reduction in IHS or the advantages of treating more patients per bed, per ward, or per year. So, the eventual benefit of a reduction in stay, is yet not documented.

Whether reducing IHS is beneficial to the patient is ambiguous. Both relatives and patients may gain a great deal of pleasure from early discharge. Satisfaction of patients and their relatives may be related to length of stay and has been shown to be high for various types of day surgery. It is possible that for different groups of patients either a longer or a shorter stay may increase satisfaction. Possible complications that might occur after surgery have to be taken into consideration. Complications such as the development of thromboembolic disease or the development of hospital-acquired infection are indicated to increase with longer stay. ${ }^{57.58}$ So, a shorter stay would decrease the risk. of these complications. Furthermore, reductions in hospital stay have been shown not to have a major negative or adverse effect on health outcome.

\section{Conclusion}

While health care budgets are being reduced, the demand for health care increases more and more. ${ }^{6 x}$ The population is aging; the medical technology expands rapidly and care consumers want better quality of care. So, health care services face the challenge to becone more efficient. It is natural to focus on standardization, shortening stay and avoiding inefficiencies or costs. By improving efficiency, a higher quality of care can be rendered, waiting lists can be shortened and workload can be decreased.

Although we are able to detect, to classify and to reduce IHS, there is still a long way to go io reach a level of minimal IHS and an optimal health care (Safe, Effective, Patientcentered. Timels: Efficient \& Equitable). ${ }^{\circ}$ Even if we might never reach that level of IHS, it is important to bear this goal in mind. Only so, we can continuously improve the efficiency and ultimately the quality of health care services. But, the pros and cons have to be weighed. Therefore additional research is needed on the general implementation of measurement tools like the DAEP and research on how processes within a hospital can be improved. Mere the use of statistical process control (SPC) can be helpful. ${ }^{69}$ In order to perform time series analysis, continuous and frequent measurements of IHS have to be implemented. We documented that the DAEP valid and reliable to assess the appropriateness of hospital stay. But since there are large differences in the proportion of IHS between countries, benchmarking with other countries might be useful to assess whether IHS is measured similarly enabling a sensible comparison of the results. 
Understanding patient flow requires looking at the whole system of health care, not just in isolated units. Reducing variation in flow has been shown to improve overall patient flow. Providing patients with timely access to appropriate care is an essential element of high quality care, because when care is provided is often as important as what care is provided.

Finally, although the proportion of IHS in the Netherlands is low in relation to other countries, ${ }^{7-21,70}$ the current strains on our health care budget means that one quarter of inappropriate output within the Dutch health care is hardly affordable, 


\section{References}

1. Institute for Healthcare Improvement. Idealized design of clinical office practice. 1999. Boston. Available from: URL: http:/www.ihi.org/idealized/idcop/background asp\#Model

2. Quaethoven. P. Hospital performance and accountability. Paper $8^{\text {th }}$ European Forum on Quality Improvement in Health Care. May $15^{\text {th }}$ 2003, Bergen.

3. Black N. Appropriateness of Hospital Use: The European BIOMED Project. European Collaboration on appropriateness of Hospital Bed Use: a Commentary Int $\mathbf{J}$ Qual Health Care 1995; 7:185-186.

4. Restuccia J, Gertman P, Dayno S, Kreger B, Lehnart G. A comparative analysis of appropriateness of hospital use. Health Aff Millwood 1984; 3:130-8.

5. Grover P. Is Inappropriate Hospital Care an Inevitable Component of the Health Care System? Med Care 1991; 29(8 Suppl): AS1-4.

6. Institute of Medicine. Crossing the Quality Chasm: A New Health System for the 21 st Century. National Academy Press. 2000; Available from URL: http:/www.nap.edu/books/0309072808/html/

7. Restuccia J. The evolution of Hospital Utilization Review Methods in the United States. Int J Qual Health Care 1995; 7:253-60.

8. Apolone G, Fellin G, Tampieri E, Bonanoni E et al. Appropriateness of hospital use. Report from an Italian study. Eur I Public Health 1997; 7:34-9.

9. Baré M. Prat A, Lledo L, Asenjo M, Salleras L. Appropriateness of admissions and hospitalization days in an acute-care teaching hospital. (in French) Rev Epidém et Santé Publ 1995; 43:328-36.

10. Bentes M, Gonsalves M, Santos M, Pina E. Design and development of a Utilization Review Program in Portugal. Int J Qual Health Care 1995; 7:201-12.

11. Fellin G. Apolone G, Tampieri A, et al. Appropriateness of Hospital use: an Overvicw of ltalian Studies. Int J Qual Health Care 1995; 7:219-25.

12. Kaya S, Erdem Y, Dogrusoz S. Halici N. Reliability of a hospital utilization revien method in Turkey: Int J Qual Health care 1998; 10:53-8.

13. Kaya S, Vural G, Eroglu K, Sain G et al. Reliability and validity of the Appropriateness Evaluation Protocol in Turkey: Int J Qual Health care 2000; $12: 325-9$

14. Lang T, Liberati A, Tampieri A, et al. A European version of the appropriateness Evaluation Protocol. Goals and Presentation. Int J of Technology Assessment in Health Care 1999; 15:185-97.

15. Lorenzo S, Suñol R. An overview of Spanish Studies on Appropriateness of Hospital Use. Int J Qual Health Care 1995: 7:213-8.

16. Lorenzo S, Beech R. Lang T, Santos-Eggiman B. An experience of utilization review in Europe: sequel to a BIOMED project. Int J Qual Health Care 1999: 11:13-9.

17. Lorenzo S. Lang T. Pastor R. Tampieri A et al. Reliabilify study of the European appropriateness evaluation protocol. Int J Qual Health care, 1999; 11:419-24

18. Merom D. Shohat T. Harari G, Oren M. Green M. Factors associated with inappropriate hospitalization dass in internal medicine wards in Israel: a crossnational surves: Int J Qual Health Care 1998: 10:155-62.

19. Paldi Y. Porath A, Friedman L. Mozes B. Factors Associated with Inappropriate Hospitalization in Medical Wards: a Cross-sectional Study in Two University Hospitals. Int J Qual Health Care 1995; 7:261-5. 
20. Robain M, Lang T, Fontaine A, et al. Reliability and validity of the French version of the first part of the Appropriateness Evaluation Protocol (AEPf): criteria of appropriateness of hospital davs (in French). Rev Épidém et Santé Publ 1999:47:139-49.

21. Santos-Eggiman B, Paccaud F, Blanc T. Medical Appropriateness of Hospital Utilization: an Overview of the Swiss Experience. Hospital utilization reviews under field conditions: Potential and Improvements. Int J Qual Health Care 1995 . 7:227-32.

22. Mannheim L, Feinglass J, Hughes R, Martin G. Conrad K. Training House Officers to be Cost Conscious. Effects of an Educational Intervention on Charges and Length of Stay. Med Care 1990; 28:29-39.

23. Winkens R. Improving test ordering in general practice. The effects of individual feedback [thesis]. Maastricht University:1994. p. 168.

24. Paccaud F, Guillain H. Should we assess appropriateness of care in Europe? Int J Qual Health Care 1994; 6:239-43.

25. Payne S. Identifying and managing inappropriate hospital utilization: a policy synthesis. Health Serv Res 1987; 22:709-69.

26. Mozes B. Rosenblum Y, Rom L, Friedman N, Shabtai E, Porat A. Medical patients assessment protocol: a tool for evaluating the appropriateness of utilizing hospitalstay days for acute medical patients; development, reliability and applications Am J Med Qual 1996; 11:18-24.

27. Hall D. Bytheway B. The blocked bed; definition of a problem. Soc Sci Med 1985; 16: 1985-91.

28. Hermans E, Anten H, Diederiks J, Philipsen H. Long-term results and substiturion among 'blocked bed' patients suffering from CVA (in Dutch). Tijdschrift voor gezondheidswetenschappen 2000: 5:275-9.

29. Pearson S, Kleefeld S, Soukop J, Cook E, Lee T. Critical pathwass intervention to reduce length of hospital stay Am J Med 2001; 110:175-80

30. Van Steenbergen W. Optimalisering van een diagnostisch pad. leterus van onbekende oorsprong. Acta hospitalia 2000; 1:41-54

31. Weingarten S, Riedinger M. Sandhu M, Bowers $C$ et al Can practice guidelines safely reduce hospital length of stay? Results from a multicenter interventional sfudy. Am J Med 1998; 150; 33-40

32. Wennberg J. Giving patients a bigger sav in choosing treatment. Med Econ. 1993 Oct 11:70: 138-42

33. Eraker S, Politser P. How decisions are reached: physician and patient Annals of Internal Medicine 1982; 97: 262-8

34. Kasper J, Mulley A, Wennberg J. Developing shared decision-making programs to improve the quality of health care. Quality Review Bulletin 1992; 18: 183-90

35. Mulley A. Supporting the patient 's role in decision-making. Journal of Occupational Medicine 1990; 12:1127-8

36. Berwick D. A primer on leading the improvement of systems. British Medical Journal 1996:312:619-622 (9 March).

37. Institute for Health Care Improvement: Optimizing Patient Flow: Moving Patients Smoothly Through Acute Care Settings 2003 Available from URL: http:/www.qualityhealthcare.org/qhe/newslettergateway.aspx?target=/QHC/Topics/ Improvement Methods/Literature/OptimizingpatientflowMovingpatientssmoothly throughacutecaresettings.htm\& contentld=D03E00CBB I C8994DB4B96A0F AAF:A 23960444) 
38. Litvak E. Maximizing Hospital Flow for Efficient and Effective Care. Boston University School of Management. 2003, Available from URL: http:/www.qualityhealthcare.org/qhc/newslettergateway.aspx?target=/QHC/Topics/ ImprovementMethods/Literature/Improvement TipTrySchedulingHospital Discharges. htm\&contentId=D03E00CBB IC8994DB4B96A0FAAEA23960444

39. Rutten C, Klunder E, de Gier D, Post. D, Smelt W. Poliklinisch preventief onderzoek door de anesthesioloog. Bedrijfseconomische evaluatie (in Dutch) Med contact 1996; 51:1142-6

40. Blauwkuip I, Rutters A. Gat in de marki. Particuliere diagnostische centra willen eigen status. (in Dutch) Med Contact 2003; 58: 862-4.

41. Miller I, Miller M. Statistical methods for quality with applications to engineering \& management. Prentice-Hali Inc. Englewood Cliffs 1995, ISBN 0-13-013749-9

42. Rozich J, Resar R. Using a unit assessment tool to optimize patient flow and staffing in a community hospital.Jt Comm J Qual Improv. 2002; 28:31-41.

43. Litvak E, Long M. Cooper A. McManus M. Emergency department diversion: causes and solutions. Acad Emerg Med. 2001; 8:1108-10.

44. Dautzenberg P. Bremer R, Lormans, M Tanis J.Ontslagvertraging in het algemeen ziekenhuis. Een oriënterend onderzoek in 's Hertogenbosch. (in Dutch) Med contact $1997 ; 42: 1310-3$

45. Braveman P, Kessel W. Egerter S, Richmond J. Early discharge and evidence-based practice. Good science and good judgement. JAMA1997; 278:334-6

46. Houghton $\mathrm{A}$ et al. Does a dedicated discharge coordinator improve the quality of hospital discharge? Qual in Health care 1996: 5:89-96

47. Vlugt, van der M. et al. Vroeg ontslag na een hartinfarct. (in Dutch) Hartbulletin 1990: 27:62-6

48. Murray M, Tantau C. Redefining open access to primary care. Manag Care Q. 1999; 7:45-55.

49. Adler M. Changes in local clinical practice following an experiment in medical care. Epidemiol Community Health 1978; 32:1436.

50. Adler M. Waller J, Kasap H, King C. Thorne S. A randomised controlled trial of early discharge for inguinal hernia and varicose veins: some problems of methodologi: Med Care 1974:12:541-7.

51. Adler M. Waller J, Creese A. Thome S. Randomised controlled trial of early discharge for inguinal hernia and varicose veins. J Epidemiol Community Health $1978 ; 32: 136-42$.

52. Health technology case study. Variations in hospital length of stay- - their relationship to health outcomes Washington. DC: US Congress, Office of Technology Assessment, 1983. (OTAHCS-23.)

53. McPherson K. Length of stay and health outcome. BMJ 1984; 88:1854-5.

54. Beech R. Withey C. Morris R. Understanding variations in lengths of stay between hospitals for fractured neck of femur patients and the potential consequences of reduced stay targets. J Public Health Med 1995: 17:77-84.

55. Jonsson B, Lindgren B. Five common fallacies in estimating the economic gains of early discharge. Soc Sci Med 1980; 14:27-33.

56. Hollingworth W, Todd C, Parker M, Roberts J, Williams R. Cost analysis of early discharge after hip fracture. BMJ 1993; 307;903-7.

57. Thromboembolic Risk Factors (THRIFT) Consensus Group. Risks of and prophylaxis for venous thromboembolism in hospital patients. BMJ 1992: 305:56774. 
58. Broderick A, Mori M. Mettleman M, Streed S, Wenzel R. Nosocomial infections: validation of surveillance and computer modeling to identify patients at risk. Am J Epidemiol 1990; 131:734-42.

59. Ruckley C. Day care and short stay surgery for hernia. Br J Surg 1978; 65:14.

60. Morris D, Ward A. Handyside A. Early discharge after hernia repair. Lancet 1968; i: $681-5$.

61. Russell I, Fell M, Devlin H, et al. Day care surgery for hernias and haemorrhoids. Lancet 1977; i: 844-7.

62. Cleary P. Greenfield S, Mulley A. Pauker S, Schroeder S, Wexler L, McNeil B. Variations in length of stay and outcomes for six medical and surgical conditions in Massachusetts and California. JAMA 1991: 266:73-9.

63. Flood A, Ewy W. Scott R, Forrest V, Brown B. The relationship between intensify and duration of medical services and outcomes for hospitalised patients. Med Care 1979; 17:1088-102.

64. Michaels J Recee-Smith H. Faber R. Cost-control study of patient satisfaction with day-case and inpatient inguinal hernia repair. J R Coll Surg Edinb 1992; 37: 99100 .

65. Thomas H, Hare MJ. Day case laparoscopic sterilization - time for a rethink? Br J Obstet Gynaecol 1987; 94:445-8.

66. Black N, Sanderson C. Day surgery - development of a questionnaire for eliciting patients' experience. Quality in Health Care 1993; 2:157-61.

67. Black N, Petticrew M, Hunter D. Sanderson C. Day surgeny development of a national comparative audit service. Quality in Health Care 1993; 2:162-7.

68. Statistics Netherlands: Statline, the statistical database on the Netherlands cited $2001 \mathrm{Jul}$ 18. Available from: URL http:/argon2.cbs.nl/statweb/index ENG.stm

69. Wortman B. The Quality Engineer Primer. Quality Council of Indiana, Sixth Edition Update, Aug 6. 1999.

70. McDonagh M, Smith D, Goddard M. Measuring appropriate use of acute beds. A systematic review of methods and results. Health Policy 2000; 53:157-84 


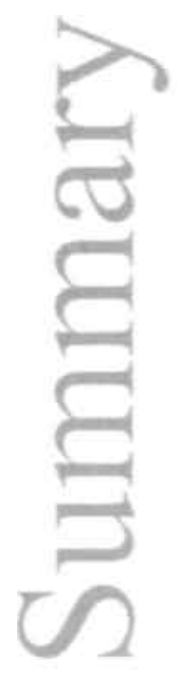

\section{Introduction}

Every year a growing amount of knowledge, procedures and techniques to improve health care, based on careful scientific research becomes available. But since the resources in health care are limited, choices concerning the allocation of people, time, facilities, equipment and knowledge have to be made. The reduction of hospital beds increases the pressure to use the remaining beds as efficiently as possible. Inefficiency in this affects the quality of hospital care and impedes the logistical patient llow, resulting in delays in patient turnover and longer waiting lists and waiting times. The increasing demands in face of limited resources, puts hospitals under great pressure to improve the efficiency of internal operations, without sacrificing quality of care. Complex systems, like health care, are best organized first by their purposes. In the case of health care the definition of this purpose must be seen through the eyes of the informed patient: "They give me exactly the help I want (and need) exactly when I want (and need) it." Since there is evidence that within health care there is often an underuse, overuse or misuse of care, health care systems should be reinvented to foster innovation and improve the delivery of care, in order to reach optimal health care. This health care is: Safe, Effective, Patient-centered, Timely, Efficient and Equitable. Appropriate care not only concerns medical procedures, but also the setting in which that care is provided. Appropriate hospital care can be seen as the match between the patient's clinical characteristics, the services required for this care and the setting in which this care is provided. Reducing inappropriate hospital stay (IHS) can reduce costs and increase efficiency, while maintaining the quality of care. But consequently the question arises: "How to measure IHS?" and "What interventions to reduce IHS are needed?". Therefore, the central objective of this thesis was formulated as: "Is the patients" stay in an acute care hospital appropriate and how can it be measured in a valid and reliable way?" 


\section{Chapter 2}

Two concepts of appropriateness have to be explained: appropriateness of care and the appropriateness of the setting in which that care is provided. The appropriateness of care is related to effectiveness and means that the expected benefits to a patient outweigh the expected harms to that patient. Hereby the procedure is labeled as "necessary" if it must -for reasons of ethics and medical necessity- be offered to the patient. The appropriateness of the setting in which care is provided is related to cost-effectiveness. This type of appropriateness is determined by whether the patient's clinical characteristics, and the services required for his or her care, match the setting in which the care is provided. When appropriateness of setting is considered, it is assumed that the services are appropriate and are provided in a technically correct way. An inappropriate setting (i.e. inappropriate hospital utilization) can be defined as utilization that is not suitable to the patient's medical need. There are two types of inappropriate utilization. Overutilization is care that is of no benefit to the patient or care that could be provided in a less costly setting. Underutilization is care that is not sufficient to meet the patient's medical need. Good health care should resonate with professional's values, but also convey a patient focus and brings in the idea of quality methods also reducing waste and increasing efficiency. A definition of quality, which many professionals have found to work best, is the three-dimensional definition of a patient, professional and management quality:

- Patient quality is what patients say they want

- Professional quality is what professionals think patients need (outcome and process)

- Management quality is using the fewest resources to give patients what they want and need, without waste, errors or delay and within policy and legal regulations

Two other concepts are important; cost containment and quality assurance. The most desirable situation is where quality improvement and cost containment meet, reducing quality waste. If quality improvement goes together with a rise in costs, the question remains if quality improvement can be justified by an increase in costs. So cost-benefit. analyses are indicated But another important question remains: which degree of cost reduction does not yield an unacceptable loss of quality!?

\section{Chapter 3}

Several factors affect hospital stay. The patient's clinical condition is a very important. factor, but not the only one. Several other factors affect (the length and appropriateness of) hospital stay. It is important to distinguish between demand factors (i.e. the need for care) and supply factors (i.e the provision of the care). If supply factors predominate. methods of practice and managerial constraints to reduce length of might be indicated. Supply factors are closely related to the setting of care and consequently the appropriateness of care. Since this thesis focuses on the setting of care (supply factors), the demand factors are only discussed briefly in this chapter. Supply factors have influence on how long a patient stays in a hospital. Bed supply seems to have the strongest associations with hospital stay. Literature shows that if there are facilities, they will be used. This may lead to overuse and this overuse may lead to misuse as it may harm the patient. Therefore, the patient should only stay in the hospital as long (i.e. short) as medi-cally required. 


\section{Chapter 4}

A literature review was performed, focusing on the validity and reliability of the Appropriateness Evaluation. Protocol (AEP) together with possibilities for intervention in order to use this tool to assess the necessity of hospital stay at the University Hospital of Maastricht. Also the first results of pilot studies. with a Dutch version of the AdultMedical AEP (days of stay) are reported here. Face, content, and convergent validity, and reliability (in terms of overall or specific agreement and by kappa) was assessed. Literature review revealed limitations in the AEP as a general and truly valid and reliable instrument for assessing the necessity of hospital stay. In applying a Dutch version, we encountered similar difficulties. Based on literature review and own findings, we concluded that the validity and reliability of the different versions of the AEP were at that time not yet up to standard. Regarding the results of the interventions thus far, we recommended additional improvements to both the instrument and the review conditions.

\section{Chapter 5}

The adult-medical AEP was modified into a valid and reliable instrument for use in the Dutch health care system. The IHS was assessed in a cross-sectional survey using a modified, Dutch version of the Appropriateness Evaluation Protocol (DAEP). The results showed that over $20 \%$ of the hospital stay was inappropriate. Half of the inappropriate hospital stay $(45.1 \%)$ was due to (internal) hospital procedures. The DAEP proved to be valid $(K=0.76 ; 95 \%$ confidence interval $(95 \% \mathrm{CI}): 0.68-0.84)$, reliable $(\mathrm{K}$ $=0.84: 95 \% \mathrm{CI}: 0.75-0.93$ ) and easy to use. A substantial proportion of hospital stay was found to be inappropriate, due to hospital procedures and the inability to refer patients to other care facilities or care providers. The DAEP can be used for monitoring the appropriate hospital stay and in detecting possible causes of inappropriate stay. Analysis of the causes of inappropriate hospital stay provided useful data for improvement actions.

\section{Chapter 6}

Extended day care (EDC) is a one-day admission spending one night in hospital. Many EDC patients do not need hospital care over night, so probably they could be transferred to a day surgery setting, resulting in decreased costs and increased efficiency. So, the appropriate length of extended day care (ALED) and a possible transfer to day surgery was assessed. ALED was defined as the time between the start of the surgical procedure and the final moment appropriate hospital care was provided. About $80 \%$ of the patients. could possibly have been treated in day surgery. The other patients could not be iransferred, because a prolonged ALED. With the implementation of new policies on admission to and discharge from the hospital and the use of altered types of operation room scheduling or patient logistics the transfer of most EDC patients to day surgery would be possible. 


\section{Chapter 7}

Traditionally, venous thromboembolism was treated in a hospital setting. Nowadays, low molecular weight heparin preparations allow most deep venous thrombosis (DVT) patients to benefit from home therapy. Chapter 7 evaluates if the previous treatment of deep venous, thrombosis in a hospital setting was appropriate in the context of modern opinion, using the DAEP. If so, the DAEP could be used to assess the appropriateness of the present hospital stay of other patient groups. So, a retrospective research of patients treated during 1995 - 1998 for DVT or pulmonary embolism (PE) (before implementation of ambulatory treatment) was conducted, assessing the appropriateness of the patient's stay using the DAEP. Only $27.1 \%$ of the treatment for DVT was found to be appropriate in a hospital setting and related to specific hospital care. The inappropriate stay was mostly related to delays in diagnostic and discharge procedures. Of the patients with PE, $50.2 \%$ needed hospital stay. This proportion was statistically significantly higher than in DVT patients ( $\mathrm{p}:<.001$ ). The extent of the DVT was not related to the length of bed rest prescribed. The DAEP was able to identify inappropriate hospital stay in the past within DVT patient. Further exploration for the potential of the DAEP to identify patient groups who possibly could be treated at home is warranted.

\section{Chapter 8}

The reasons for IHS and possible predictors of IHS were identified and assessed. The reasons for IHS were analyzed in a cross-sectional survey at two surgical, one gynecologic and one obstetric ward. Results indicate that more than $20 \%$ of the hospital stay was inappropriate. The reasons for IHS were statistically significantly related to the patients' age, the availability of home care and specialism. In a predictive model, only the specialism proved to be a predictor of IHS. Most of the IHS occurred during the first days of hospital stay and the days before the patient's discharge. A substantial proportion of hospital stay was found to be inappropriate, due to hospital procedures and the inability to refer patients to other care facilities or care providers. Analysis of the causes of IHS provided useful data for improvement actions. Efficient use of hospital resources should be promoted by reducing the delay in interventions and discharge procedures.

\section{Chapter 9}

The reduction of hospital beds, the ageing of the population and the increased demand for hospital admissions requires the provision of the right care in the right setting. Since hospital stay should be as short and as appropriate as possible and previous studies showed a large proportion of the stay to be inappropriate, often related to delays in hospital procedures, the objective was to decrease IHS related to delays in diagnostic procedures and to hospital discharge within the department of Internal Medicine. Additionaily, control charts were used to monitor the processes of hospital discharge and diagnostics and to differentiate between normal and abnormal variance. To reduce the delay in diagnostic procedures 'slots" (i.e. reserved time for diagnostic procedures) were set up. To reduce the delay in hospital discharge an 'admission \& discharge team' was introduced. The IHS related to both delays in hospital discharge and diagnostic procedures decreased by $3.4 \%$. However, only the actions to improve the process of hospital discharge were significantly successful. P-charts showed the variation of this process to remain within the control limits. The IHS related to the unavailability of other health care facilities increased by $6.1 \%$. Specific hospital procedures have been improved. However, the impact of these improvement actions was undone by the increase of IHS caused by the unavailability of other health care facilities. 


\section{Chapter 10}

Appropriate hospital stay should be: effective, efficient and tailored to the patients' needs. Within Obstetrics inappropriate hospital stay (IHS) consisted mostly of delays in hospital discharge. The specific goals of chapter 10 were: reducing IHS by fine-tuning the patient logistics; increasing the efficiency and providing a more comforting surrounding. New discharge policies using strict discharge criteria were implemented. Thereby, the mothers could leave the hospital in an earlier stage and use an external family accommodation, near the hospital. The total IHS decreased from 13.3 to $7.2 \%$. The delay in discharge procedures halved. P-charts showed a decreasing fluctuation in the IHS, indicating the current process to become more stable. A significant reduction of IHS by the implementation of innovative hospital discharge policies was found, indicating a higher efficiency and accessibility of hospital services.

\section{Chapter 11: Lessons learned}

The core business of health care is to provide the right care at the right time. Combining quality and efficiency should be the second core-business in health care, so that, given a certain budget, the best possible care is rendered. To do so, a systematic approach of planning, implementation, control and (re-)action is required. People may hold different views about, inappropriate health care delivery, but as a matter of fact, health care that does not benefit the patient should be avoided. And the health care that is needed should not be provided in an inappropriate setting. An inappropriate setting means that care could be provided in an alternative setting (usually on an outpatient basis or at home) at a lower cost. However, the verb "could" should be emphasized; the required care may not be available in an alternative setting. In this, the patient's interest (and preferences) should prevail. But, the question remains if the aim should be a reduction of IHS per se (i.e. quality \& efficiency improvement) or that a reduced IHS should be put against the means that are needed to reach the goals (i.e. cost - benefit analysis). The ultimate goal of a 'lean production' (zero IHS) may not always be the best option. Although we are able to detect, to classify and to improve IHS, there is still a long way to go to reach the level of minimal or 'zero IHS' on one hand a an optimal health care on the other hand as defined by the Institute of Medicine (Safe; Effective, Patient-centered, Timely: Efficient \& Equitable). Even if we might never reach that level of IHS, it is important to bear this goal in mind. Only so, we can continuously improve the efficiency and ultimately the quality of health care services. But, the pros and cons have to be weighted. Therefore additional research is needed on the general implementation of measurement tools like the DAEP' and research on how processes within a hospital can be improved. Here the use of statistical process control (SPC) can be helpful. But in order to perform time series analysis, continuous and frequent measurements of IHS have to be implemented. We proved the DAEP valid and reliable to assess the appropriateness of hospital stay, but since there are large differences in the proportion of IHS between countries, bench marking with these countries can be useful, in order to assess if the way of measuring IHS is the same, so the results can be compared. As final remark; Considering the strains on health care budgets, and although the proportion of IHS is low in relation to other countries, one quarter of inappropriate output within the Dutch health care is hardly affordable. 


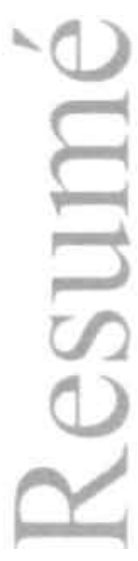

\section{Inleiding}

Jaarlijks komt een steeds grotere hoeveelheid aan kennis, procedures en technieken ier verbetering van de gezondheidszorg beschikbaar. Echter, door de beperkte middelen, dienen er keuzes gemaakt te worden omtrent het inzetten van mensen, middelen en materialen. Ziekenhuisbedden moeten zo efficiënt mogelijk gebruikt worden om wachttijden en wachtlijsten tot een minimum te beperken. Complexe systemen zoals de gezondheidszorg worden het best georganiseerd m.b.t. hun doelstellingen. Het standpunt van de patiënt hierin is: "Ik krijg de zorg die ik wil (en nodig heb) op het ogenblik dat ik het wil (en nodig heb)". Omdat er in de gezondheidszorg kans bestaat op overgebruik, ondergebruik of zelfs misbruik van voorzieningen, zou deze zorg herontworpen moeten worden teneinde een optimale gezondheidszorg te verwezenlijken. Dit zou betekenen: 'Veilig, Effectief, Efficiënt, Patiënt-georiënteerd, Tijdig en Onpartijdig'. Noodzakelijke zorg slaat niet alleen op (medische) zorgverlening, maar ook op de setting waarin deze zorg verleend wordt. Noodzakelijke zorg is de overeenkomst tussen de zorgbehoefte van de patiënt, de hieraan gerelateerde zorg en de setting waarin deze zorg verleend wordt. Het verminderen van het niet noodzakelijk (ziekenhuis)verblijf (NNV) kan de kosten beperken en de efficiëntie verhogen terwijl de kwaliteit van zorg gewaarborgd blijft. Maar hierbij is de vraag: "Hoe kan het NNV gemeten worden?" en "Welke interventies moet men invoeren om het NNV te verminderen?". Daarom werd de centrale vraag in dit proefschrift als volgt geformuleerd: "Is het verblijf van een patiënt in het ziekenhuis noodzakelijk en hoe kan dit op een valide en betrouwbare manier gemeten worden?". 


\section{Hoofdstuk 2}

Ter beoordeling van de noodzaak van het klinische verblijf zijn twee concepten van belang: de noodzaak van de zorgverlening en de noodzaak van de setting waarin die zorgverlening plaatsvindt. De noodzaak van de zorgverlening is gerelateerd aan effectiviteit. Hierbij primeren de verwachte voordelen over de mogelijke nadelen van een behandeling. Hierbij wordt de zorgverlening als noodzakelijk gezien als deze om ethische of medische redenen verleend moet worden. De noodzaak van de setting van de zorgverlening is gekoppeld aan kosteneffectiviteit en wordt bepaald door bepaalde patientenkenmerken en de mate waarin deze overeenkomen met de setting. Hierbij wordt verondersteld dat de zorgverlening goed is in kwalitatief, technisch en professioneel opzicht. Goede zorgverlening kan het best omschreven worden in een driedimensionale definitie van kwaliteit, gerelateerd aan kenmerken op het gebied van patiënt, zorgverlener en organisatie:

- Patiëntgerelateerde kwaliteit is wat de patiënt zegt dat hij wil;

- Professionele kwaliteit is wat de zorgverlener denk dat de patiënt nodig heeft (resultaat en proces);

- Organisatie gerelateerde kwaliteit is het gebruik van zo weinig mogelijk middelen om de patiënt te geven wat hij wil en nodig heeft, zonder verspilling, fouten of vertragingen, met in acht neming van bestaande regelgeving.

Hierbij zijn twee andere concepten van belang: kostenbeheersing en kwaliteitsbewaking. De meest wenselijke situatie is als kwaliteitsverbetering gepaard gaat met kostenvermindering. Indien een kwaliteitsverbetering gepaard gaat met een stijging van de kosten, moet men zich afvragen of dit gerechtvaardigd kan worden in termen van kosteneffectiviteit. Hetzelfde geldt als kostenbesparingen leiden tot kwaliteitsverlies. Hierbij blijft steeds de vraag: "Wat is acceptabel en wat niet?".

\section{Houfdstuk 3}

Hel verblijf in een ziekenhuis word beïnvloed door verschillende factoren. De medische conditic van een patiënt is zeer belangrijk, maar het is niet de enige factor. Het is belangrijk vraag- en aanbodfactoren te onderscheiden (zorgbehoefte vs. zorgverlening). Dit proefschrift richt zich met name op de aanbodfactoren. Aanbodfactoren beïnvloeden de lengle van het ziekenhuisverblijf. Hierbij speelt vooral het beddenaanbod een grote rol. Literatuuronderzoek toont aan dat als er voorzieningen beschikbaar zijn, ze hoe dan ook gebruikt zullen worden. Dit leidt tot overgebruik en zelfs misbruik indien dit schadelijk zou zijn voor de patiënt. Daarom is het van belang dat het verblijf in een ziekenhuis zo kort als medisch verantwoord is, dient te zijn.

\section{Hoofdstuk 4}

Tijdens de literatuurstudie ging de aandacht uit naar de validiteit en betrouwbaarheid van het Appropriateness Evaluation Protocol (AEP). Hierbij werd ook onderzocht of dit instrument geschikt was om de noodzaak van het klinisch verblijf van patiënten in het academisch ziekenhuis Maastricht (azM) te beoordelen. Ook de resultaten van een vooronderzoek met een Nederlandse versie van het AEP worden in dit hoofdstuk gepresenteerd inclusief resultaten m.b.t. validiteit en betrouwbaarheid. Literatuuronderzoek liet een aantal beperkingen van het AEP zien als algemeen instrument ter beoordeling van de noodzaak van het ziekenhuisverblijf. Ook het gebruik van de Nederlandse versie van dit instrument toonde gelijkaardige problemen. Op basis van deze gegevens kon geconcludeerd worden dat bijkomende aanpassingen aan het instrument en in de wijze van gebruik noodzakelijk: waren. 


\section{Hoofdistuk 5}

De validiteit en betrouwbaarheid van de aangepaste versie van het Nederlandse AEP (DAEP) wordt verder getest en bevestigd (validiteit: $\kappa: 0.76$, betrouwbaarheid: $\kappa: 0.84$ ). De noodzaak van het klinisch verblijf in het azM wordt d.m.v. een transversale steekproef onderzocht. Hieruit blijkt dat meer dan $20 \%$ van het ziekenhuisverblijf als niet noodzakelijk bestempeld kan worden. Bijna de helft van dit NNV $(45,1 \%)$ is gerelateerd aan processen in het ziekenhuis. De andere helft van het NNV is hoofdzakelijk gerelateerd aan 'verkeerde bed problematiek'. Uit dit onderzoek bleek dat het DAEP gebruikt kan worden om NNV vast te stellen en de oorzaken hiervan op te sporen. Analyse van de redenen van NNV leverde bruikbare informatie op voor specifieke verbeterprojecten.

\section{Hoofdstuk 6}

Vierentwintig-uurs opnames (VUO) zijn dagopnames waarbij de patiënt een nacht in het ziekenhuis verblijft. Veel van deze patiënten hebben 's nachts geen ziekenhuiszorg nodig. Deze patiënten zouden dus ook via een dagcentrum behandeld kunnen worden, hetgeen een kostenbesparing en een verhoging van de efficiëntie zou inhouden. Hiertoe werd de noodzakelijk duur van VUO's (NDV) en een eventuele overplaatsing van deze patięnten naar een dagcentrum onderzocht. NDV werd hierbij gedefinieerd als de tijdsperiode tussen begin van de ingreep en het ogenblik waarop voor het laatst zorgverlening in het ziekenhuis nodig was. Op basis van de onderzoeksgegevens kon geconcludeerd worden dat $80 \%$ van deze patiënten via een dagcentrum behandeld zou kunnen worden. Door het invoeren van een aangepast opname- en ontslagbeleid en aangepaste operatieschema's of patiëntenlogistiek zou een overplaatsing van het merendeel van deze patiënten naar een dagcentrum mogelijk zijn.

\section{Hoofdstuk 7}

Tot voor kort werden veneuze trombo-embolieën in een ziekenhuis behandeld. Tegenwoordig kunnen door het gebruik van heparine preparaten met een laag moleculair gewicht de meeste patiënten met een diep veneuze trombose (DVT) thuis behandeld worden. We wilden nagaan of het DAEP in staat was de noodzaak van het ziekenhuis verblijf tijdens behandeling in het verleden vast te stellen in het licht van hedendaagse opvattingen over deze behandeling. Mocht dit mogelijk blijken, zou het gebruik van het DAEP uitgebreid kunnen worden naar andere patiëntencategorieën. Hiertoe werd in een retrospectief onderzoek de noodzaak van het ziekenhuisverblijf van patiënten die van 1995 tot 1998 voor DVT of longembolie (LE) behandeld waren, onderzocht. Siechts in $27,1 \%$ bleek voor de behandeling van DVT ziekenhuisverblijf noodzakelijk te zijn. Het NNV was vooral gerelateerd aan vertragingen in diagnostiek en ontslag. Tijdens de behandeling van LE bleek in 50,2\% van de gevallen ziekenhuisopname noodzakelijk. De hoogte van DVT bleek niet gerelateerd aan de lengte van de voorgeschreven bedrust. 


\section{Hoofdstuk 8}

De redenen yoor NNV en mogelijke voorspellende factoren hiervoor werden verder onderzocht en geanalyseerd. De gevonden proporties van het NNV blijken overeen te komen met eerdere onderzoeken in het azM. De redenen van het NNV zijn statistisch significant gerelateerd aan de leeftijd van de patiënt, de beschikbaarheid van zorgverlening in de thuissituatie en het medisch specialisme. In een voorspellend model (logistische regressie) blijkt alleen het medisch specialisme een statistisch significante voorspellende factor voor NNV te zijn. Het NNV treedt vooral op tijdens de eerste dagen na de opname van een patiënt en de dagen voorafgaand aan het ontslag van de patiënt uit het ziekenhuis. Ook hier blijkt het NNV gerelateerd aan bestaande ziekenhuisprocessen en een gebrek aan overplaatsingsmogelijkheden van patiënten naar andere instellingen.

\section{Hoofdstuk 9}

De reductie van ziekenhuisbedden, het vergrijzen van de bevolking en de verhoogde vraag naar ziekenhuiszorg vergen een optimaal gebruik van de resterende bedden. waarbij de juiste zorg op de juiste plek verleend wordt. Aangezien het verblijf in een ziekenhuis zo kort en zo noodzakelijk mogelijk dient te zijn en voorgaand onderzoek een substantieel deel aan NNV liet zien -vaak gerelateerd aan processen binnen het ziekenhuis-, was de doelstelling bij dit gedeelte van het onderzoek de vertragingen tijdens diagnostiek en tijdens de ontslagprocedure binnen het medisch specialisme Interne Geneeskunde te verminderen. Als bijkomend controlemiddel werden regelkaarten (P-kaarten) gebruik om abnormale varianties binnen deze processen te kunnen onderscheiden. Ter vermindering van vertragingen tijdens de diagnostiek werden slottijden ingevoerd. Om vertragingen tijdens de ontslagprocedure te verminderen werden 'opname- en ontslagteams' gevormd. Het NNV veroorzaakt door bovenvermelde oorzaken daalde door deze mastregelen met $3,4 \%$. Echter, alleen de verbetering in het ontslagproces bleek vantit statistisch significant oogpunt een succes. Bij dit proces bleef de variantie binnen de regelgrenzen. Echter het NNV veroorzaakt door factoren buiten het ziekenhuis (verkeerde bed problematiek) steeg met $6,1 \%$.

\section{Hoofdstuk 10}

Noodzakelijk zickenhuisverblijf kañ betiteid worden als: effectief. efficięn en aangepast aan de behoeften van de patiënt. Echter een groot gedeelte van dit verblijf blijkt niet noodzakelijk te zijn. Binnen het specialisme Obstetrie bestaat het NNV hoofdzakelijk uit vertragingen in de ontslagprocedure, die op hun beurt weer veroorzaakt worden door de opname van de pasgeborene(n). Ter vermindering van dit. NNV werden de volgende doelen gesteld: het stroomlijnen van de patiëntenlogistiek, het verhogen van de efficièntie en het verlenen van de noodzakelijke zorg in een. comfortabele omgeving, terwijl de moeder toch in de nabjiheid van het kind kon zijn. Hiertoe werden een nieuw ontslagbeleid en -criteria ingevoerd. Hierdoor konden moeders het ziekenhuis eerder verlaten en gebruik maken van her Ronaid McDonaldhuis. Hierdoor daalde het totale NNV van 13,3 naar $7.2 \%$ en halveerden de vertragingen in de ontslagprocedure. Regelkaarten toonden een afnemende variantie en een stabilisering van het proces. 


\section{Hoofdstuk 11}

De kernactiviteit van de gezondheidszorg is het verlenen van geschikte zorg op het geschikte tijdstip. Het samenbrengen van kwaliteit en efficiëntie zou de tweede kernactiviteit van de gezondheidszorg moeten zijn, zodat, binnen een bepaald budget, de best mogelijke zorg wordt verleend. Dit vereist een systematische planning, implementatie, beheersing en (re-)actie. Men kan uiteenlopende standpunten innemen over noodzakelijke gezondheidszorg. maar zorg die niet ten goede komt aan de patiěnt moet vermeden worden. En de noodzakelijk e zorg moet op een geschikte plaats (setting) verleend worden. Een ongeschikte setting houdt in dat de zorg vaak goedkoper in een alternatieve setting verleend zou kunnen worden. Echter de benodigde zorg kan (nog) niet beschikbaar zijn in die alternatieve setting. Hierbij dienen echter altijd de belangen (en de voorkeuren) van de patiënt voorop te staan. De vraag blijft wat het doel moet zijn: cen absolute vermindering van het NNV (kwaliteits- en efficiěntieverbetering) of dat deze vermindering afgezet moet worden tegen de benodigde middelen en inspanningen (kostenbatenanalyse). Het ultieme doel van een 'productie zonder verspilling' (geen NNV) hoeft niet de beste oplossing te zijn. Hoewel we in staat zijn NNV op te sporen, te classificeren en re verbeteren, is er nog een hele weg te gaan om een optimale gezondheidszorg te bereiken (Veilig. Effectief. Efficiënt. Patiënt-georiënteerd. Tijdig en Onpartijdig) Zelfs als we dit doel niet bereiken, is het belangrijk hiernaar te blijven streven. Alleen op deze wijze kan de kwaliteit en de efficiëntie van de gezondheidszorg continu verbeterd worden. Hierbij moeten echter de voor- en nadelen afgewogen worden. Hiertoe is aanvullend onderzoek nodig naar een algemene invoering van instrumenten als het DAEP en hoe processen in het ziekenhuis verbeterd kunnen worden. Hierbij kan statistische proces beheersing een goed hulpmiddel zijn. Echter om trendanalyses te kunnen uitvoeren zijn continue en frequente metingen m.b.t. het NNV noodzakelijk.

Dit proefsehrift toont aan dat het DAEP valide en betrouwbaar instrument is om de noodzaak van het ziekenhuisverbliff te beoordelen. Echter gezien de grote verschillen in percentage van NNV zowel bimen als buiten een organisatie, zou bench marking nuttig zijn om meetwijzen te kunnen vergelijken, zodat ook de resulaten vergeleken kunnen worden. Als laatste opmerking kan gesteld worden dat, gezien de strakke budgetten van de gezondheidszorg, een $20 \%$ niet efficiënte productie nauwelijks geoorloofd mag worden. 


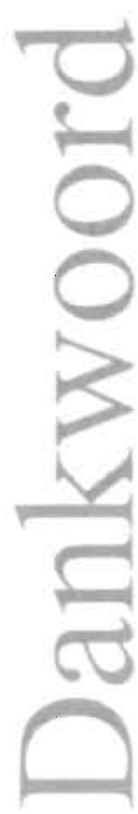

Mijn speciale dank gaat uit naar hen die als promotor of co-promotor optraden;

Martin, zoals reeds eerder gezegd, ben jij je gewicht in goud waard. Alhoewel we er beiden van overtuigd zijn dat dit een kostbare aangelegenheid zou kunnen worden, is het uitgangspunt hiervan verschillend; jij refereert aan het metrieke stelsel en ik aan de (professionele) waarde. Ik vind dat onze Filet Americain best geproefd mag worden. Ik zorgde voor de biefstuk. Jij maakte er vervolgens gehakt van. Maar jouw kruidige kritiek, bracht het tenslotte op smaak.

Frank, je motiveerde me steeds weer tot nieuwe uitdagingen. Eén hiervan was het ontcijferen van je handschrift en dit ondanks mijn 15-jarige ervaring met doktershandschriften. Echter jouw opmerkingen en commentaar waren zeer waardevol en belichtien vaak een andere kant van het probleem. Hierdoor wonnen het onderzoek en de artikels zeker aan waarde. Ik wil je ook bedanken voor de tijd (in de trein en buiten kantooruren) die je eraan besteed hebt.

Peter, jouw jarenlange ervaring binnen Interne Geneeskunde en het Transmuraal \& Diagrostisch Centrum waren van onschatbare waarde bij het opzetten en tot stand komen van dit proefschrift, waarvoor mijn oprechte dank. Vooral jouw kritische benadering van het medisch handelen was zeer waardevol bij het bediscussiëren van de verschillende hoofdstukken

Ik wil alle collega's vạn de afdeling KEMTA bedanken voor de fijne samenwerking, de goede werksfeer, hun vaak zeer bruikbare tips en voor de tijd dat ze mijn gezeur wilden moesten aanhoren. Vooral Brigitte was hier het slachtoffer van. Desondanks hebben we vijf jaar zonder al te veel kleerscheuren dezelfde kamer kunnen delen. Ook je in- 
breng bij de correctie van correspondentie was zeer waardevol. Dank zij Fred ben ik nu ook op de hoogte van de schrikreactie van Rigel XVI ("It's a perfectly bodily function and it is odourless"). Ooit zal ik mijn belofte inlossen een strippenkaart in "au Coin' vol (leeg) te maken. Verder ben ik iedereen dankbaar dat ik na afloop van het project enige tijd als getolereerde, illegale allochtoon binnen KEMTA mocht verblijven.

Daarnaast gaat mijn speciale dank uit naar alle leidinggevenden en verpleegkundigen van de afdelingen waar data verzameld zijn. Ondanks het feit dat het een bijkomende belasting voor jullie was, hebben jullie de steeds de moeite genomen deze data te verzamelen. Zonder jullie was dit proefschrift nooit tot stand gekomen. Deze dank gaat ook uit naar de managementteams van de verschillende Behandel- en Zorgeenheden waaronder bovengenoemde afdelingen vallen. Zonder instemming en medewerking van deze teams had het onderzoek nooit opgestart en afgerond kunnen worden.

Chantal, bedankt voor je ontwerp van de omslag van dit proefschrift. Dit maakt het tot een blijvend aandenken.

Beste schoonouders, ik wil mijn waardering uitspreken voor jullie belangstelling en steun in de soms belastende (schrijf)tijden.

Lieve ouders, het verheugt me dat jullie allebei deze gebeurtenis mogen meemaken. Mede door jullie ben ik geworden tot wie ik ben. Daar ben ik jullie zeer dankbaar voor.

Lieve Wouter en Emma, dankzij jullie kan ik het leven naar waarde schatten en is thuis Thuis. Dat vind ik geweldig.

Liẹste Eliçe, ik kạn moẹilijk joụw aạdẹel in mijn lẹven beschrijven. Misschịen kan ịk dit het beste uitleggen door te zeggen dat ik mijn koffer net zo goed kan inpakken als jij. Maar jij vergeet mijn tandenborstel tenminste niet!! Dus Elice, zonder jou... 


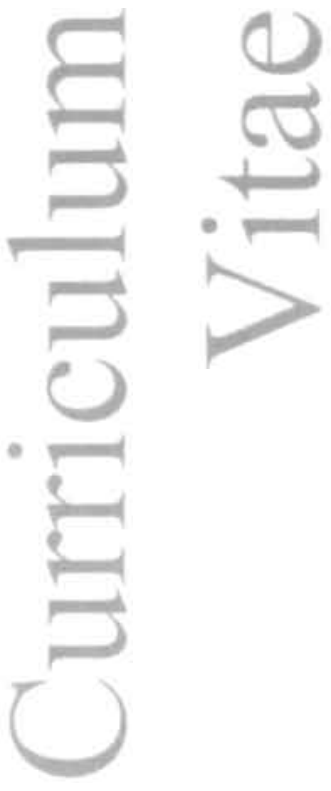

Bert Panis werd geboren op 5 april 1963 te Hasselt (Belgię). In 1981 behaalde hij het eindexamen 'Oude Humaniora" (Latijn-Grieks) aan het Heilig Hartcoliege te Lanaken. In 1986 voltooide hij de opleiding tot verpleegkundige in het ziekenhuis St. Annadal te Maastricht. Tot 1998 werkte hij op verschillende verpleegafdelingen, waarvan meer dan 10 jaar op een Neonatale Intensive Care Unit (NICU). Hierbij specialiseerde hij zich van 1988 tot 1991 tot kinderverpleegkundige en ICN-verpleegkundige (Intensive Care Neonatologie). Vervolgens startte hij in deeltijd met de studie Gezondheidswetenschappen aan de Rijksuniversiteit Limburg waar hij in 1994 afstudeerde met als specificatie Verplegingswetenschappen en afstudeerrichting Beleid \& Beheer. Sinds 1997 werkt hij als KRAM-coördinator (Kwaliteit, Risico, ARBO \& Milieu) voor Behandel- en Zorgeenheid II van het academisch ziekenhuis Maastricht (azM). In 2000 werd hij door het ASQ (American Society for Quality Control) gecertificeerd als Certified Quality Engineer (CQE). Daarnaast gaf hij van 2001 tot 2003 leiding aan de polikliniek Kindergeneeskunde en de Kinderprikdienst van het azM. Van 1998 tot 2003 werkte hij ook als onderzoeker voor de afdeling Klinische Epidemiologie \& Medical Technology Assessment (KEMTA) van het azM, belast met het onderzoek naar de noodzaak van het klinische verblijf van patiënten in het ziekenhuis. 


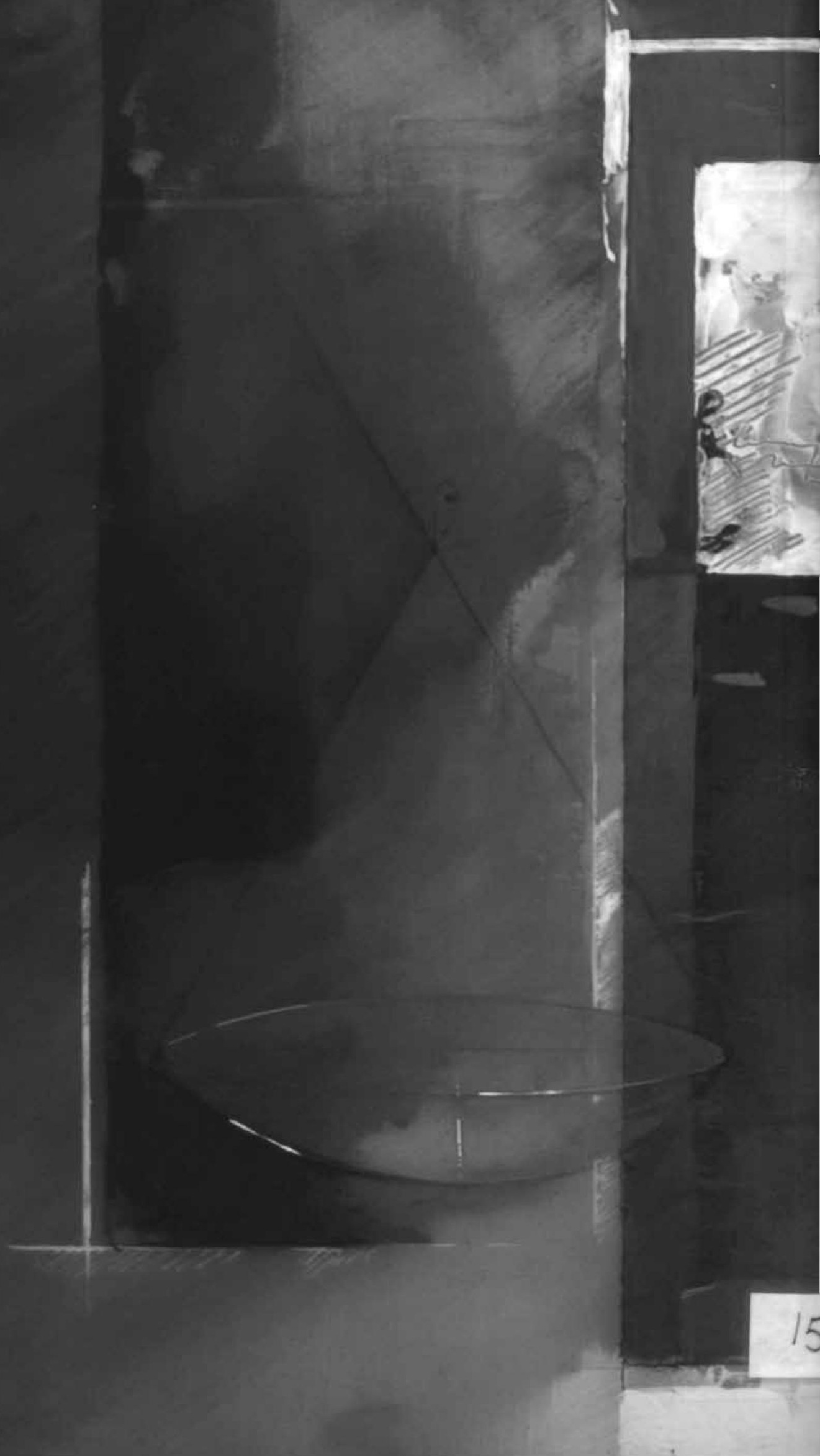

